\title{
Perovskite-type superlattices from lead halide perovskite nanocubes
}

\section{Journal Article}

\section{Author(s):}

Cherniukh, Ihor; Rainò, Gabriele; Stöferle, Thilo; Burian, Max; Travesset, Alex; Naumenko, Denys; Amenitsch, Heinz; Erni, Rolf (D); Mahrt, Rainer F.; Bodnarchuk, Maryna I.; Kovalenko, Maksym V.

Publication date:

2021-05-27

\section{Permanent link:}

https://doi.org/10.3929/ethz-b-000488424

\section{Rights / license:}

In Copyright - Non-Commercial Use Permitted

\section{Originally published in:}

Nature 593(7860), https://doi.org/10.1038/s41586-021-03492-5

Funding acknowledgement:

192308 - Q-Light - Engineered Quantum Light Sources with Nanocrystal Assemblies (SNF) 


\title{
Perovskite-type superlattices from lead-halide perovskite nanocubes
}

\author{
Ihor Cherniukh ${ }^{1,2}$, Gabriele Rainò ${ }^{1,2}$, Thilo Stöferle ${ }^{3}$, Max Burian ${ }^{4}$, Alex \\ Travesset $^{5}$, Denys Naumenko ${ }^{6}$, Heinz Amenitsch ${ }^{6}$, Rolf Erni ${ }^{7}$, Rainer F. Mahrt ${ }^{3}$, \\ Maryna I. Bodnarchuk ${ }^{1,2}$, Maksym V. Kovalenko ${ }^{1,2 *}$
}

\footnotetext{
${ }^{1}$ Department of Chemistry and Applied Bioscience, Institute of Inorganic Chemistry, ETH Zürich, Zürich, Switzerland.

${ }^{2}$ Laboratory of Thin Films and Photovoltaics, Empa - Swiss Federal Laboratories for Materials Science and Technology, Dübendorf, Switzerland.

${ }^{3}$ IBM Research Europe - Zurich, Rüschlikon, Switzerland.

${ }^{4}$ Swiss Light Source, Paul Scherrer Institut, Villigen PSI, Switzerland.

${ }^{5}$ Department of Physics and Astronomy, Iowa State University and Ames Lab, Ames, Iowa, USA.

${ }^{6}$ Institute of Inorganic Chemistry, Graz University of Technology, Graz, Austria.

${ }^{7}$ Electron Microscopy Center, Empa - Swiss Federal Laboratories for Materials Science and Technology, Dübendorf, Switzerland
} 
For over 80 years, tailored molecular assemblies (e.g., H- and J-aggregates) have been of interest for the emergence of collective phenomena in their optical spectra ${ }^{1}$, coherent long-range energy transport ${ }^{2,3}$ and their conceptual similarity with natural light-harvesting complexes ${ }^{4,5}$. Another highly versatile platform for creating controlled, aggregated states exhibiting collective phenomena arises from the organization of colloidal semiconductor nanocrystals (NCs) into long-range ordered superlattices (SLs) ${ }^{6}$. Cesium lead halide perovskite $\mathrm{NCs}^{7-9}$ have recently emerged as highly appealing building blocks, owing to their high oscillator strength ${ }^{10}$, slow dephasing (long coherence times of up to $80 \mathrm{ps})^{11,12}$, minimal inhomogeneous broadening of emission lines, and a bright triplet exciton character with orthogonal dipole orientation ${ }^{10}$, potentially enabling an efficient omnidirectional coupling. Here we present perovskite-type $\left(\mathrm{ABO}_{3}\right)$ binary and ternary NC SLs by a shapedirected co-assembly of steric-stabilized, highly luminescent cuboid-shaped $\mathrm{CsPbBr}_{3} \mathrm{NCs}$ (occupying B- and/or O-sites) with spherical $\mathrm{Fe}_{3} \mathrm{O}_{4}$ or $\mathrm{NaGdF}_{4} \mathrm{NCs}$ (A-sites) and truncated-cuboid PbS NCs (B-site). Such $\mathrm{ABO}_{3} \mathrm{SLs}$, as well as other newly obtained SL structures (binary NaCl- and $\mathrm{AlB}_{2}$-types), exhibit a high degree of orientational ordering of $\mathrm{CsPbBr}_{3}$ nanocubes. These novel perovskite mesostructures exhibit superfluorescence (SF) - a collective emission resulting in a burst of photons. SF is characterized, at high excitation density, by emission pulses with ultrafast (22 ps) radiative decay and Burnham-Chiao ringing behaviour with a strongly accelerated build-up time. 
SF, unlike fluorescence, is a collective emission of several, incoherently photo-excited dipoles mediated by their common photon field, and is characterized by faster radiative lifetimes and the appearance of Burnham-Chiao ringing behaviour ${ }^{13}$. Previously, these features could be realized either in the gaseous state (HF gas) ${ }^{14}$ or in a limited number of solid-state systems (CuCl-doped $\mathrm{NaCl}$, peroxide-ion-doped $\mathrm{KCl}$, and epitaxial InGaAs quantum wells) ${ }^{15-17}$. SF in lead halide perovskite NC SLs was recently demonstrated with the simplest packing geometry - simple cubic packing (scp) of cubic NCs into a 3D-supercrystal ${ }^{13}$. Far broader structural engineerability of SLs, required for programmable tuning of the collective emission and for building a theoretical framework, can be envisioned from the recent advancements in colloidal science ${ }^{6,18,19}$.

The formation of NC SLs is generally governed by the balance between the enthalpic contributions and entropic interactions ${ }^{6}$. Enthalpy prevails when, for instance, specific pair-wise interactions between NCs are designed, such as in the aqueous-based co-assembly of DNA-coated noble metal NCs with DNA linkers ${ }^{20,21}$, which is not an applicable strategy to water-degradable perovskite NCs. However, entropy can become a prevailing factor for steric-stabilized apolar NC colloids; that is, when the strong short-range repulsion of hydrocarbon ligand chains is the principle component of the interparticle potential, causing a hard-sphere-like behaviour of NCs. Such colloids often undergo a transition into the densest possible periodic arrays upon solvent evaporation, hexagonal close packing $(h c p)$ or facecentred cubic ( $f c c)$ for spheres ${ }^{22}$ or $s c p$ for cubes (see Ref. ${ }^{23}$ and, for perovskite NCs, also Ref. ${ }^{13,24-27}$ ), maximizing the free-volume entropy of the system ${ }^{6}$. Furthermore, mixtures of spherical NCs were shown to form at least twenty different binary SL structures, usually those with higher packing density for a given ratio of their effective hard-sphere diameters. Typically, SLs that are isostructural with $\mathrm{NaCl}, \mathrm{NaZn}_{13}, \mathrm{AlB}_{2}, \mathrm{MgZn}_{2}$ and $\mathrm{CaCu}_{5}$ are observed, Rather surprisingly, there have been no reports on the formation of multicomponent SLs comprising steric-stabilized cubic NCs. For instance, in the case of a NaCl-type SL, a mixture of larger spheres (A) and smaller cubes (B) will yield greater packing density $(\eta)$ than that of two $(\mathrm{A}+\mathrm{B})$ spheres at any B/A size ratio $\left(\gamma=d_{B} / d_{A}<1\right.$, Fig. $1 \mathrm{k}$ and Supplementary Note 1).

In this work, we not only show that co-assembly of cubic and spherical steric-stabilized NCs is experimentally possible, but also that the cubic shape of perovskite NCs leads to a vastly different outcome of such assembly, as compared to all-spherical systems. Specifically, we present perovskite-type $\left(\mathrm{ABO}_{3}\right)$ binary and ternary NC SLs (Figs. 1, 2, 4, 5, Extended Data Figs. 1, 2, 4-7 and Supplementary Figs. 7-12). In binary SLs, larger spherical $\mathrm{Fe}_{3} \mathrm{O}_{4}$ or $\mathrm{NaGdF}_{4} \mathrm{NCs}$ occupy the A-sites and smaller cubic $\mathrm{CsPbBr}_{3}$ NCs reside on both B- and O-sites, whereas in ternary SLs the B-sites 
are occupied by truncated-cuboid PbS NCs. We have also obtained NaCl-type SLs (combining $5.3 \mathrm{~nm} \mathrm{CsPbBr}_{3}$ with 14.7-19.8 $\mathrm{nm} \mathrm{Fe}_{3} \mathrm{O}_{4} \mathrm{NCs}$, and $8.6 \mathrm{~nm} \mathrm{CsPbBr}$ with 10.2-25.1 $\mathrm{nm} \mathrm{Fe}_{3} \mathrm{O}_{4}$ and $18.6 \mathrm{~nm} \mathrm{NaGdF} 4 \mathrm{NCs}$, see Fig. 3 and Supplementary Figs. 13, 14) and $\mathrm{AlB}_{2}$-type SLs (in mixtures of $5.3 \mathrm{~nm} \mathrm{CsPbBr} 3$ with $12.5 \mathrm{~nm} \mathrm{Fe} \mathrm{O}_{4} \mathrm{NCs}$, and $8.6 \mathrm{~nm}$ $\mathrm{CsPbBr}_{3}$ with $19.8 \mathrm{~nm} \mathrm{Fe}_{3} \mathrm{O}_{4} \mathrm{NCs}$, Extended Data Fig. 3). Importantly, cubic perovskite NCs exhibit a high degree of orientational order within all observed binary SLs. We then demonstrate that these novel perovskite-based mesostructures exhibit SF.

The non-truncated (sharp) cuboid shape of $\mathrm{CsPbX}$ NCs - their decisive property for obtaining $\mathrm{ABO}_{3} \mathrm{SLs}-$ arises from the pseudo-cubic faceting of the underlying orthorhombic perovskite lattice as well as various specifics of surface termination and ligand binding (see Ref. ${ }^{30}$ and Supplementary Note 2). In order to prevent a sphere-like behaviour in the NC assembly caused by the softening of the cubic NC shape by long-chain ligands typically used for the NC synthesis $^{31}$, the shortest-chain capping ligand that is still capable of both the efficient colloidal stabilization and retention of high photoluminescence (PL) quantum yield, namely didodecyldimethylammonium bromide (DDAB) ${ }^{30}$, was used. The softness $(\lambda)$ of a NC can be defined by the ratio of the doubled capping ligand length to the NC diameter ( $d_{\text {core }}$ ), or the edge length for cubic NCs $\left(l_{\text {core }}\right)$ (i.e., $\lambda=2 L / d_{\text {core }}$ and $\lambda=2 L / l_{\text {core }}$, respectively). Monodisperse $\mathrm{CsPbBr}_{3}$ NCs of two sizes, $5.3 \mathrm{~nm}(\lambda=0.63)$ and $8.6 \mathrm{~nm}(\lambda=0.39)$, were synthesized as reported previously ${ }^{30,32}$ [see transmission electron microscopy (TEM) images and optical characterization in Supplementary Figs. 5 and 6, respectively]. Trials of colloidal crystallization were conducted by controlled solvent evaporation from the mixture of NCs in toluene on various substrates. The structure of the observed SL was characterized with high-angle annular darkfield scanning TEM (HAADF-STEM) at various tilt angles, electron tomography, elemental mapping with energydispersive X-ray spectroscopy (EDX-STEM), grazing-incidence small-angle X-ray scattering (GISAXS), scanning electron microscopy (SEM), as well as electron diffraction (ED, wide-angle) and small-angle ED.

\section{Binary SLs}

$\mathrm{ABO}_{3}$-type SLs (isostructural with cubic $\mathrm{CaTiO}_{3}$ perovskite, $\mathrm{Pm} \overline{3} m$ space group) had thus far not been observed in the colloidal crystallization of steric-stabilized NCs. In this lattice, $\mathrm{CsPbBr}_{3} \mathrm{NCs}$ occupy two different Wyckoff positions: $1 \mathrm{~b}$ (i.e., B-site, with the $<100>$ crystallographic directions of the nanocubes aligned with $<100>_{\text {sL; }}$; the subscript "SL" denotes the Miller indices of the SL) and 3c (i.e., O-site, two $<110>$ aligned with $<100>_{\mathrm{SL}}$ ). Firstly, $8.6 \mathrm{~nm} \mathrm{CsPbBr}_{3}$ NCs were combined with oleate-capped $\mathrm{Fe}_{3} \mathrm{O}_{4} \mathrm{NCs}$ (9.6-25.1 nm, Supplementary Fig. 5). Experiments with 9.6-14 nm $\mathrm{Fe}_{3} \mathrm{O}_{4}$ NCs yielded separate phases of single-component SLs or disordered mixtures, except for small NaCl-type 
domains. With 14.5-20.7 nm Fe $\mathrm{O}_{4} \mathrm{NCs}$, however, large domains of $\mathrm{ABO}_{3}$-type SLs were observed (up to $\sim 100 \mu \mathrm{m}^{2}$; Fig. 1 and Supplementary Fig. 7). $\mathrm{ABO}_{3}$-type SLs were also obtained by combining $8.6 \mathrm{~nm} \mathrm{CsPbBr}_{3}$ nanocubes with 15.2-19.5 nm NaGdF 4 NCs (Fig. 5, Extended Data Fig. 2).

Columns of $\mathrm{CsPbr}_{3}$ nanocubes are readily resolved in HAADF-STEM and TEM images as well as from EDX-STEM maps of [001 $]_{\mathrm{SL}-}$-oriented domains (e.g., Fig. 1 and Extended Data Fig. 1). In particular, Z-contrast makes the heavyelement containing $\mathrm{CsPBBr}_{3}$ NCs brighter in HAADF-STEM images. EDX-STEM maps also assist in the visualization of the frame of perovskite NCs, showing occupancy of every SL unit cell facet by the O-site nanocube. HAADF-STEM images taken at different tilting angles and compared with simulated projections readily differentiate the binary $\mathrm{ABO}_{3}$ lattice from CsCl- and $\mathrm{Li}_{3} \mathrm{Bi}$-types (Fig. 2e, $\mathrm{f}$ and Supplementary Figs. 8, 9), but do not unambiguously exclude the $\mathrm{Cu}_{3} \mathrm{Au}$-type packing. Full clarity as to occupancy and orientation of the nanocubes was then obtained with ED patterns and respective dark-field TEM images for specific diffraction spots (Fig. 2a-d). The $\mathrm{Fe}_{3} \mathrm{O}_{4}$ NCs exhibit random orientations, apparent from a broad diffraction ring corresponding to the (311) lattice planes. On the contrary, a high degree of orientational order of the $\mathrm{CsPBr}_{3} \mathrm{NCs}$ is readily seen as intense arcs corresponding to diffraction on (100), (110), and (200) planes, which are normal to $\langle 100\rangle_{\mathrm{SL}}$ and $\langle 110\rangle_{\mathrm{SL}}$. Additionally, the (111) reflections are split into eight maxima with a splitting angle of $\sim 19.5^{\circ}$ (reflections "9" and "10" in Fig. 2b). This angle corresponds to the theoretical value for a pair of $\mathrm{CsPbBr}_{3}$ cubes located on two orthogonal facets and oriented such that their faces are in contact with the spheres (blue and yellow cubes in Fig. 2c). The centre cubes (B-sites) are rotated $45^{\circ}$ with respect to those on the O-sites and hence exhibit distinct reflections (labelled "2" and "4", Fig. 2b). These site occupancies are also confirmed by the dark-field TEM images (Fig. 2d), constructed from the diffraction spots originating from B- or O- positioned cubes ("2,4" and "1,3,6,8,9,10", respectively) or from both ("5,7"). Electron tomography of a small isolated domain further confirms the $\mathrm{ABO}_{3}$ structure and visualizes the orientation of $\mathrm{B}$ - and O-site cubes (Supplementary Fig. 10 and Supplementary Video 1). Domains exhibiting [102 $]_{\text {SL, }}[111]_{\text {SL }}$ and [112 $]_{\text {SL }}$ orientations were also observed (Supplementary Fig. 11). GISAXS measurements show strong, periodic reflections (Fig. 1c), evidencing a high degree of both in-plane and out-of-plane order. The 2D diffraction pattern corresponds to a simplecubic symmetry $(\operatorname{Pm} \overline{3} m)$ with a $24.2 \mathrm{~nm}$ unit cell (Supplementary Note 3). The in-plane coherent scattering volume, corresponding to the ordered domain size, is $>360 \mathrm{~nm}$ (inset in Supplementary Fig. 4a). No structural phase transitions were observed by TEM and ED upon cooling to liquid nitrogen temperature (Supplementary Fig. 12). 
With smaller $5.3 \mathrm{~nm} \mathrm{CsPbBr} 3$ NCs, which can be considered as "rounded" cubes in the view of their higher softness parameter $\lambda$, no $\mathrm{ABO}_{3}$-type lattice was observed; rather, lattices that are commonly known for binary mixtures of hard spheres (NaCl- and $\mathrm{AlB}_{2}$-types, Supplementary Fig. 13 and Extended Data Fig. 3b-d, respectively). For $8.6 \mathrm{~nm}$ $\mathrm{CsPbBr} 3$ combined with 15.6-19.8 nm $\mathrm{Fe}_{3} \mathrm{O}_{4} \mathrm{NCs}$, the three $\mathrm{ABO}_{3}$, $\mathrm{AlB}_{2-}$ (Extended Data Fig. 3e, f) and NaCl-type (Fig. 3) SLs compete. The proportion of $\mathrm{AlB}_{2}$ - and NaCl-type SLs increases with decreasing perovskite NC particle fraction in the mixture. When the size of A-site spheres is further increased to $25.1 \mathrm{~nm}$, the NaCl-type SL is favoured (Supplementary Fig. 14).

The stability of the binary SLs follows from the analysis of $\eta$ as a function of $\gamma$. The effective sizes of the NCs used for computing $\gamma$ are calculated by taking into account both the dimension of the inorganic NC core and the ligand shell thickness $^{33} \cdot \eta$ is then calculated following the hard particle formalism, assuming that the ligand shell is space-filling only along the axis connecting two NCs (Optimal Packing Model, OPM) and, in addition, considering the deformability of the ligand shell, namely the bending of ligands away from the axis of contact between NCs (Orbifold Topological Model, OTM) ${ }^{34,35}$. For low coordination numbers, OTM predicts higher $\eta$ and smaller lattice parameters, in good agreement with experimental values ${ }^{29}$. All-sphere binary SL structures with OPM packing densities above 0.65, sometimes even exceeding the $f c c / h c p(\eta=0.74)$, are commonly observed ${ }^{6,29}$. The corresponding range of $\gamma$-values is $\sim 0.3-0.65$. Very few reports demonstrate SLs at higher $\gamma$-values (mainly $\mathrm{CaCu}_{5}$ - or $\mathrm{MgZn}_{2}$-types at $\gamma=0.65-0.86$ ) $28,36,37$. Interestingly, the OPM packing densities of NaCl-type SLs with cubes on the B-site are $>0.705$ across the entire $\gamma$ range, unlike all-sphere NaCl-type SLs that are stable and usually observed only at $\gamma<0.5$ (Fig. 1k). Indeed, grains of NaCl-type SLs were observed for $\mathrm{CsPbBr}_{3}$ NCs co-assembled with $\mathrm{Fe}_{3} \mathrm{O}_{4}$ NCs for $\gamma=0.27-0.73$ (Fig. 3 and Supplementary Figs. 13, 14). Analysis of ED and small-angle ED again reveals the high degree of orientational order of perovskite NCs in a NaCl-type binary SL; for instance, the $\mathrm{CsPbr}_{3}<100>$ directions coincide with $<100>_{\mathrm{SL}}$.

Although the formation of an $\mathrm{ABO}_{3}$-like SL had not been reported and not expected for hard spheres (Fig. 1k), it readily forms with $\mathrm{CsPbBr}_{3}$ nanocubes at $\gamma=0.399,0.420,0.477,0.486,0.509$ and 0.541 . OPM calculations (Fig. 1k) can only explain the first two examples. At $\gamma \leq \gamma_{c, 1}=0.414$, the assembly comprises an $s c p$ lattice of A spheres; cubes can partially rattle in-between, but cannot freely rotate. At $\gamma>\gamma_{c, 1}$, the shape of the cubes becomes a stronger factor. The cubes are in contact with the spheres (via their facets) and with each other (via sharp vertices) and, according to OTM, ligand vortices form (Supplementary Fig. 2). The overall effect of the cubic shape and ligand deformability is the locking of the cube orientations for both the B- and O-sites as well as much higher packing densities. The OTM 
thus explains the formation of $\mathrm{ABO}_{3}$-type SLs in a broader $\gamma$-range (see the OTM-branch at $\gamma>0.414$ in Fig. $1 \mathrm{k}$ ). The validity of the OTM results is also evident from the better correspondence of the OTM-predicted lattice parameters with the experimental values (Supplementary Table 2 and Supplementary Note 1).

\section{Ternary $\mathrm{ABO}_{3}$-type SL}

The obtained binary $\mathrm{ABO}_{3} \mathrm{SLs}$ should not be confused with a perovskite-derived $\mathrm{Ni}_{4} \mathrm{~N}$-type due to the non-equivalent orientation of the B- and O-positioned cubes. Furthermore, the centre cube (B-site) also differs in terms of coordination environment. In the case of large spherical A-site NCs, especially at $\gamma=\gamma_{c, 1}$, the space available for B- and O-cubes is different because O-cubes are in contact with A-spheres, while B-cubes are still rattlers (have freedom to displace themselves around their equilibrium position). This intuition motivated us to explore the possibility of a targeted occupation of the B-site by a larger NC (compared to the O-site), thus yielding a ternary $\mathrm{ABO}_{3}$-type SL. For example, the case of a truncated B-site cube with the degree of truncation $\tau=0.835$ at $\gamma_{O / A}=\gamma_{c, 1}=0.414$ and $\gamma_{B / A}=0.586$ corresponds to a simultaneous appearance of A-O, A-B, and B-O contacts and a high OPM-packing density of $\eta=0.918$, greatly exceeding the density of a binary lattice at $\gamma=0.414(\eta=0.808)$. Experimentally, this was realized herein by combining $10.7 \mathrm{~nm}$ truncated cubic PbS NCs with $8.6 \mathrm{~nm}$ cubic $\mathrm{CsPbBr}_{3}$ and $19.8 \mathrm{~nm}$ spherical $\mathrm{Fe}_{3} \mathrm{O}_{4} \mathrm{NCs}$ $\left(\gamma_{O / A}=0.414, \gamma_{B / A}=0.533\right.$; Fig. 4, Extended Data Figs. 4, 5). The appearance of B-site PbS NCs is evident from their higher contrast in TEM and HAADF-STEM images in the [001 $]_{\mathrm{SL}},[111]_{\mathrm{SL}},[101]_{\mathrm{SL}}$ and $[112]_{\mathrm{SL}}$ orientations. EDXSTEM maps further confirm the incorporation of PbS NCs onto B-sites. Combined ED and small-angle ED analysis indicates that the orientation of the O-site cubes in the ternary SL is the same as in binary SLs. The degree of B-site substitution, as evidenced by ED, is adjustable by increasing the $\mathrm{PbS}: \mathrm{CsPbBr}_{3} \mathrm{NC}$ mixing ratio (Fig. 4 and Extended Data Fig. 6).

Atomic perovskite lattices exhibit remarkable structural and compositional engineerability and a close analogy to the presented NC SL system is that of $\mathrm{CaTi}_{(1-x)} \mathrm{Mn}_{\mathrm{x}} \mathrm{O}_{3}$ - a B-site solid solution preserving the perovskite Pnma symmetry. Larger A-site cations such as $\mathrm{Ba}^{2+}$, however, cause phase segregation even at $\mathrm{x}=0.01$, driven by the instability of the perovskite phase $\mathrm{BaMnO}_{3} 38,39$ as the Goldschmidt tolerance factor exceeds the stability range of $0.8-1^{40}$. Within this understanding, we set to increase the size of the A-site $\mathrm{Fe}_{3} \mathrm{O}_{4} \mathrm{NCs}$ to emulate this Ca-to-Ba transition. In cases of larger $\mathrm{Fe}_{3} \mathrm{O}_{4} \mathrm{NC}$ sizes of $21.5 \mathrm{~nm}\left(\gamma_{B, O / A}=0.387, \eta=0.755\right)$ and $25.1 \mathrm{~nm}\left(\gamma_{B, O / A}=0.337, \eta=0.677\right)$ combined with $8.6 \mathrm{~nm}$ $\mathrm{CsPbBr} 3 \mathrm{NCs}$, the OPM packing densities of binary $\mathrm{ABO}_{3}$ SLs were found to be reduced; seen as a reduced propensity to form SLs (small domains of $<0.25 \mu \mathrm{m}^{2}$, ca. $1 \%$ of SL yield). The addition of $11.7 \mathrm{~nm} \mathrm{PbS} \mathrm{NCs}\left(\gamma_{B / A}=0.537\right.$ and 
$\gamma_{B / A}=0.469$, respectively) led to ternary $\mathrm{ABO}_{3}$-type SLs in high yield and with complete substitution on the B-site (see Extended Data Figs. 6e, f and 7). Thus far, very fewer examples of ternary SLs had been serendipitously observed for spherical sterically-stabilized NCs $\left(\mathrm{AlMgB}_{4}-\mathrm{type}^{41}\right.$ and bilayered $\mathrm{ABC}_{2}$-type $\left.{ }^{42}\right)$. An overarching challenge is to outcompete crystallization into unary and binary SLs. While the formation of multinary solid-state compounds is governed by chemical bonding, NC self-assembly relies on vastly different interactions, wherein shape-anisotropy plays an increasingly important role for obtaining diverse structures, exemplified by the observed strong formability of $\mathrm{ABO}_{3}$-type SLs as well as ABC-type columnar SLs formed by the mixture of disk- and rod-shaped $\mathrm{NCs}^{43}$. Preliminary studies combining disks (oleate-capped $\mathrm{LaF}_{3}$ ) and $\mathrm{CsPbBr}_{3}$ nanocubes yielded six columnar binary SLs, not observed in the disks-spheres mixtures (Extended Data Fig. 8).

\section{Superfluorescence from $\mathrm{ABO}_{3}$-type SLs}

In molecular aggregates, the weak and broad spectral features of the individual molecules evolve into intense and narrow lines, which are shifted in energy and exhibit enhanced (or suppressed) radiative rates for J-aggregates (or Haggregates, respectively) $)^{44,45}$. Perovskite NCs emerge as an attractive mesoscale alternative for attaining collective behaviour. In particular, $8.6 \mathrm{~nm} \mathrm{CsPbBr} 3$ NCs, co-assembled with spherical $\mathrm{NaGdF}_{4} \mathrm{NCs}$ into an $\mathrm{ABO}_{3}$-type $\mathrm{SL}$ (on $\mathrm{Si}_{3} \mathrm{~N}_{4}$-membranes), exhibit coherent collective emission at low temperatures, upon pulsed excitation (Fig. 5). The emission spectrum comprises two bands (Fig. 5d), of which a narrower and redshifted (by 30 meV) band arises from coupled NCs. Fluence-dependent experiments (inset of Fig. 5d) evidence a linear dependence of the integrated PL intensity, confirming the absence of competing, power-dependent non-radiative processes (e.g., the Auger process) as well as the absence of a threshold that would be commonly associated with amplified spontaneous emission. Drastic shortening of the exciton radiative lifetime and appearance of intensity oscillations with increasing excitation fluence (Fig. 5e, f) attest to the observed emission as being SF. The long exciton coherence, high transition oscillator strength, and low energetic disorder of individual perovskite $\mathrm{NCs}^{10-12}$ enable coherent coupling of multiple transition dipole moments via a common radiation field, resulting in a single giant dipole, which leads to short and intense bursts of $\operatorname{light}^{13,46}$.

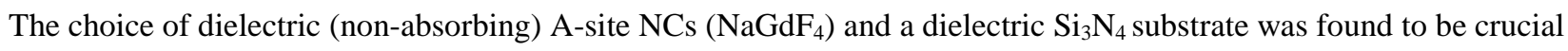
for the appearance of SF, since the presence of absorbing materials alter the exciton dynamics at high fluences, presumably by introducing non-radiative channels which quench the PL (see data with the carbon membrane as a substrate in Extended Data Fig. 9). The radiative lifetime is inversely proportional to the number of coherently coupled 
NCs, $N$, which increases with excitation fluence. Correspondingly, the peak intensity at zero-delay time increases superlinearly over more than two orders of magnitude (Fig. 5h, middle panel). Radiative lifetimes as short as 22 ps can be obtained from spectrally-integrated time-resolved PL (Fig. 5g, h) based on a phenomenological decay model ${ }^{13}$. Furthermore, a shortening of the SF build-up time, after which the photon burst is emitted, is observed (Fig. 5h). This build-up time indicates the time it takes for individual dipoles to phase-lock by interacting via the common photon field; it scales with $N$ according to $\tau_{\text {build-up }} \propto(\ln N) / N$. A good fit to experimental data can be obtained by using this theoretical expression and assuming $N$ to be proportional to the excitation fluence. Interestingly, the characteristic SF speed-up and oscillations could be retained up to $105 \mathrm{~K}$ (Fig. 5i), albeit with a higher fluence required for their emergence. This is likely the result of exciton-phonon interactions, which accelerate the exciton dephasing time, rendering the collective coupling less efficient. For example, a much slower SF build-up time is found at $105 \mathrm{~K}$ (Fig. 5i), which eventually becomes longer than the exciton dephasing time such that SF vanishes entirely at higher temperatures. $\mathrm{ABO}_{3}$-type SLs exhibit superior collective emission when compared to NaCl-type SLs (Supplementary Note 4, Extended Data Fig. 10), which we attribute to higher perovskite NC-to-dielectric NC ratio (4:1 vs. 1:1) and much reduced perovskite NC-to-NC distance (higher perovskite NC density), which facilitate the occurrence of coherent coupling. In addition, SF from $\mathrm{ABO}_{3}$-type SLs can be qualitatively altered, namely shifted from lower to higher energies compared to excitonic PL, simply by increasing the size of $\mathrm{NaGdF}_{4}$ NCs (Supplementary Note 4; Extended Data Fig. 10). We expect that further atomistic and morphological engineering of individual perovskite NC emitters (impacting the phonon spectrum, optical coherence, and exciton fine-structure) and exploitation of the vast engineerability of SLs at the mesoscale (diverse SL structures) and at microscale (SL dimensions, positioning, and device-integration) will foster important studies on many-body light-matter interactions in complex lattices such as the Dicke quantum phase transitions ${ }^{47,48}$. Such investigations may yield brighter perovskite NC assemblies or, more intriguingly, may boost the development of massively-entangled multi-photon quantum light sources ${ }^{49}$, which could find applications in optical quantum computing or quantum imaging applications ${ }^{50,51}$. 


\section{References:}

1. Spano, F. C. The spectral signatures of Frenkel polarons in $\mathrm{H}$ - and J-aggregates. Acc. Chem. Res. 43, 429-439 (2010).

2. Haedler, A. T. et al. Long-range energy transport in single supramolecular nanofibres at room temperature. Nature 523, 196-199 (2015).

3. Brédas, J.-L., Sargent, E. H. \& Scholes, G. D. Photovoltaic concepts inspired by coherence effects in photosynthetic systems. Nat. Mater. 16, 35-44 (2017).

4. Scheibe, G. Über den Mechanismus der Sensibilisierung photochemischer Reaktionen durch Farbstoffe, insbesondere der Assimilation. Naturwissenschaften 25, 795-795 (1937).

5. Franck, J. \& Teller, E. Migration and photochemical action of excitation energy in crystals. J. Chem. Phys. 6, 861-872 (1938).

6. Boles, M. A., Engel, M. \& Talapin, D. V. Self-assembly of colloidal nanocrystals: from intricate structures to functional materials. Chem. Rev. 116, 11220-11289 (2016).

7. Protesescu, L. et al. Nanocrystals of cesium lead halide perovskites ( $\mathrm{CsPbX}_{3}, \mathrm{X}=\mathrm{Cl}, \mathrm{Br}$, and I): novel optoelectronic materials showing bright emission with wide color gamut. Nano Lett. 15, 3692-3696 (2015).

8. Kovalenko, M. V., Protesescu, L. \& Bodnarchuk, M. I. Properties and potential optoelectronic applications of lead halide perovskite nanocrystals. Science 358, 745-750 (2017).

9. Akkerman, Q. A., Rainò, G., Kovalenko, M. V. \& Manna, L. Genesis, challenges and opportunities for colloidal lead halide perovskite nanocrystals. Nat. Mater. 17, 394-405 (2018).

10. Becker, M. A. et al. Bright triplet excitons in caesium lead halide perovskites. Nature 553, 189-193 (2018).

11. Becker, M. A. et al. Long exciton dephasing time and coherent phonon coupling in $\mathrm{CsPbBr}_{2} \mathrm{Cl}$ perovskite nanocrystals. Nano Lett. 18, 7546-7551 (2018).

12. Utzat, H. et al. Coherent single-photon emission from colloidal lead halide perovskite quantum dots. Science 363, 1068-1072 (2019).

13. Rainò, G. et al. Superfluorescence from lead halide perovskite quantum dot superlattices. Nature 563, 671-675 (2018).

14. Skribanowitz, N., Herman, I. P., MacGillivray, J. C. \& Feld, M. S. Observation of Dicke superradiance in optically pumped HF gas. Phys. Rev. Lett. 30, 309-312 (1973).

15. Miyajima, K., Kagotani, Y., Saito, S., Ashida, M. \& Itoh, T. Superfluorescent pulsed emission from biexcitons in an ensemble of semiconductor quantum dots. J. Phys. Condens. Matter 21, 195802 (2009).

16. Malcuit, M. S., Maki, J. J., Simkin, D. J. \& Boyd, R. W. Transition from superfluorescence to amplified spontaneous emission. Phys. Rev. Lett. 59, 1189-1192 (1987).

17. Timothy Noe II, G. et al. Giant superfluorescent bursts from a semiconductor magneto-plasma. Nat. Phys. 8, 219-224 (2012).

18. Shevchenko, E. V., Talapin, D. V., Kotov, N. A., O'Brien, S. \& Murray, C. B. Structural diversity in binary nanoparticle superlattices. Nature 439, 55-59 (2006).

19. Tan, R., Zhu, H., Cao, C. \& Chen, O. Multi-component superstructures self-assembled from nanocrystal building blocks. Nanoscale 8, 9944-9961 (2016).

20. Nykypanchuk, D., Maye, M. M., van der Lelie, D. \& Gang, O. DNA-guided crystallization of colloidal nanoparticles. Nature 451, 549-552 (2008).

21. Laramy, C. R., O'Brien, M. N. \& Mirkin, C. A. Crystal engineering with DNA. Nat. Rev. Mater. 4, 201224 (2019).

22. Murray, C. B., Kagan, C. R. \& Bawendi, M. G. Self-organization of CdSe nanocrystallites into threedimensional quantum dot superlattices. Science 270, 1335-1338 (1995). 
23. Kang, Y., Ye, X. \& Murray, C. B. Size- and shape-selective synthesis of metal nanocrystals and nanowires using CO as a reducing agent. Angew. Chem. Int. Ed. 49, 6156-6159 (2010).

24. Kovalenko, M. V. \& Bodnarchuk, M. I. Lead halide perovskite nanocrystals: from discovery to selfassembly and applications. Chimia 71, 461-470 (2017).

25. Nagaoka, Y. et al. Nanocube superlattices of cesium lead bromide perovskites and pressureinduced phase transformations at atomic and mesoscale levels. Adv. Mater. 29, 1606666 (2017).

26. van der Burgt, J. S. et al. Cuboidal supraparticles self-assembled from cubic $\mathrm{CsPbBr}_{3}$ perovskite nanocrystals. J. Phys. Chem. C 122, 15706-15712 (2018).

27. Baranov, D., Toso, S., Imran, M. \& Manna, L. Investigation into the photoluminescence red shift in cesium lead bromide nanocrystal superlattices. J. Phys. Chem. Lett. 10, 655-660 (2019).

28. Chen, Z. \& O'Brien, S. Structure direction of II-VI semiconductor quantum dot binary nanoparticle superlattices by tuning radius ratio. ACS Nano 2, 1219-1229 (2008).

29. Coropceanu, I., Boles, M. A. \& Talapin, D. V. Systematic mapping of binary nanocrystal superlattices: the role of topology in phase selection. J. Am. Chem. Soc. 141, 5728-5740 (2019).

30. Bodnarchuk, M. I. et al. Rationalizing and controlling the surface structure and electronic passivation of cesium lead halide nanocrystals. ACS Energy Lett. 4, 63-74 (2019).

31. Jishkariani, D. et al. Nanocrystal core size and shape substitutional doping and underlying crystalline order in nanocrystal superlattices. ACS Nano 13, $5712-5719$ (2019).

32. Dong, Y. et al. Precise control of quantum confinement in cesium lead halide perovskite quantum dots via thermodynamic equilibrium. Nano Lett. 18, 3716-3722 (2018).

33. Landman, U. \& Luedtke, W. D. Small is different: energetic, structural, thermal, and mechanical properties of passivated nanocluster assemblies. Faraday Discuss. 125, 1-22 (2004).

34. Travesset, A. Topological structure prediction in binary nanoparticle superlattices. Soft Matter 13, 147-157 (2016).

35. Travesset, A. Soft skyrmions, spontaneous valence and selection rules in nanoparticle superlattices. ACS Nano 11, 5375-5382 (2017).

36. Evers, W. H. et al. Entropy-driven formation of binary semiconductor-nanocrystal superlattices. Nano Lett. 10, 4235-4241 (2010).

37. Boles, M. A. \& Talapin, D. V. Many-body effects in nanocrystal superlattices: departure from sphere packing explains stability of binary phases. J. Am. Chem. Soc. 137, 4494-4502 (2015).

38. Dang, N. V., Dang, N. T., Ho, T. A., Tran, N. \& Phan, T. L. Electronic structure and magnetic properties of $\mathrm{BaTi}_{1-\mathrm{x}} \mathrm{Mn}_{\mathrm{x}} \mathrm{O}_{3}$. Curr. Appl. Phys. 18, 150-154 (2018).

39. Shi, C.-Y., Hao, Y.-M. \& Hu, Z.-B. Structural and magnetic properties of single perovskite $\mathrm{Ca}\left(\mathrm{Ti}_{1 / 2} \mathrm{Mn}_{1 / 2}\right) \mathrm{O}_{3}$. J. Magn. Magn. Mater. 323, 1973-1976 (2011).

40. Vieten, J. et al. Materials design of perovskite solid solutions for thermochemical applications. Energy Environ. Sci. 12, 1369-1384 (2019).

41. Evers, W. H., Friedrich, H., Filion, L., Dijkstra, M. \& Vanmaekelbergh, D. Observation of a ternary nanocrystal superlattice and its structural characterization by electron tomography. Angew. Chem. Int. Ed. 48, 9655-9657 (2009).

42. Dong, A., Ye, X., Chen, J. \& Murray, C. B. Two-dimensional binary and ternary nanocrystal superlattices: the case of monolayers and bilayers. Nano Lett. 11, 1804-1809 (2011).

43. Paik, T., Diroll, B. T., Kagan, C. R. \& Murray, C. B. Binary and ternary superlattices self-assembled from colloidal nanodisks and nanorods. J. Am. Chem. Soc. 137, 6662-6669 (2015).

44. Scheibe, G. Über die Veränderlichkeit der Absorptionsspektren in Lösungen und die Nebenvalenzen als ihre Ursache. Angew. Chem. 50, 212-219 (1937).

45. Jelley, E. E. Spectral absorption and fluorescence of dyes in the molecular state. Nature 138, 10091010 (1936).

46. Bonifacio, R. \& Lugiato, L. A. Cooperative radiation processes in two-level systems: superfluorescence. Phys. Rev. A 11, 1507-1521 (1975). 
47. Baumann, K., Guerlin, C., Brennecke, F. \& Esslinger, T. Dicke quantum phase transition with a superfluid gas in an optical cavity. Nature 464, 1301-1306 (2010).

48. Strack, P. \& Sachdev, S. Dicke quantum spin glass of atoms and photons. Phys. Rev. Lett. 107, 277202 (2011).

49. Munoz, C. S. et al. Emitters of N-photon bundles. Nat. Photon. 8, 550-555 (2014).

50. Wang, $\mathrm{H}$. et al. Boson sampling with 20 Input photons and a 60 -mode interferometer in a $10^{14}$ dimensional Hilbert space. Phys. Rev. Lett. 123, 250503 (2019).

51. Tenne, R. et al. Super-resolution enhancement by quantum image scanning microscopy. Nat. Photon. 13, 116-122 (2018). 
Acknowledgements This work was primarily supported by the European Union through Horizon 2020 Research and Innovation Programme (ERC CoG Grant, grant agreement No. 819740, project SCALE-HALO) and, in part, by the Swiss National Science Foundation (grant No. 200021_192308, project "Q-Light"). The authors also acknowledge the funding received from EU-H2020 under grant agreement No. 654360 supporting the Transnational Access Activity within the framework NFFA-Europe to the TUG's ELETTRA SAXS beamline of CERIC-ERIC. We thank Y. Shynkarenko for assistance with high-resolution SEM imaging, respectively.

Author contributions M.V.K., M.I.B. and G.R. conceived and supervised the project. I.C. performed the synthesis and self-assembly experiments. I.C. and R.E. characterized the materials by electron microscopy. M.B., D.N. and H.A. characterized the materials by GISAXS. G.R., R.F.M. and T.S. carried out optical measurements. A.T. contributed to packing density analysis. I.C. and M.V.K. wrote the manuscript, with input from all authors.

Competing interests The authors declare no financial or non-financial competing interests.

\section{Additional information}

Supplementary Information is available for this paper.

Materials \& Correspondence Maksym V. Kovalenko (mvkovalenko@ethz.ch)

Correspondence and requests for materials should be addressed to M.V.K.

Reprints and permissions information is available at http://www.nature.com/reprints 

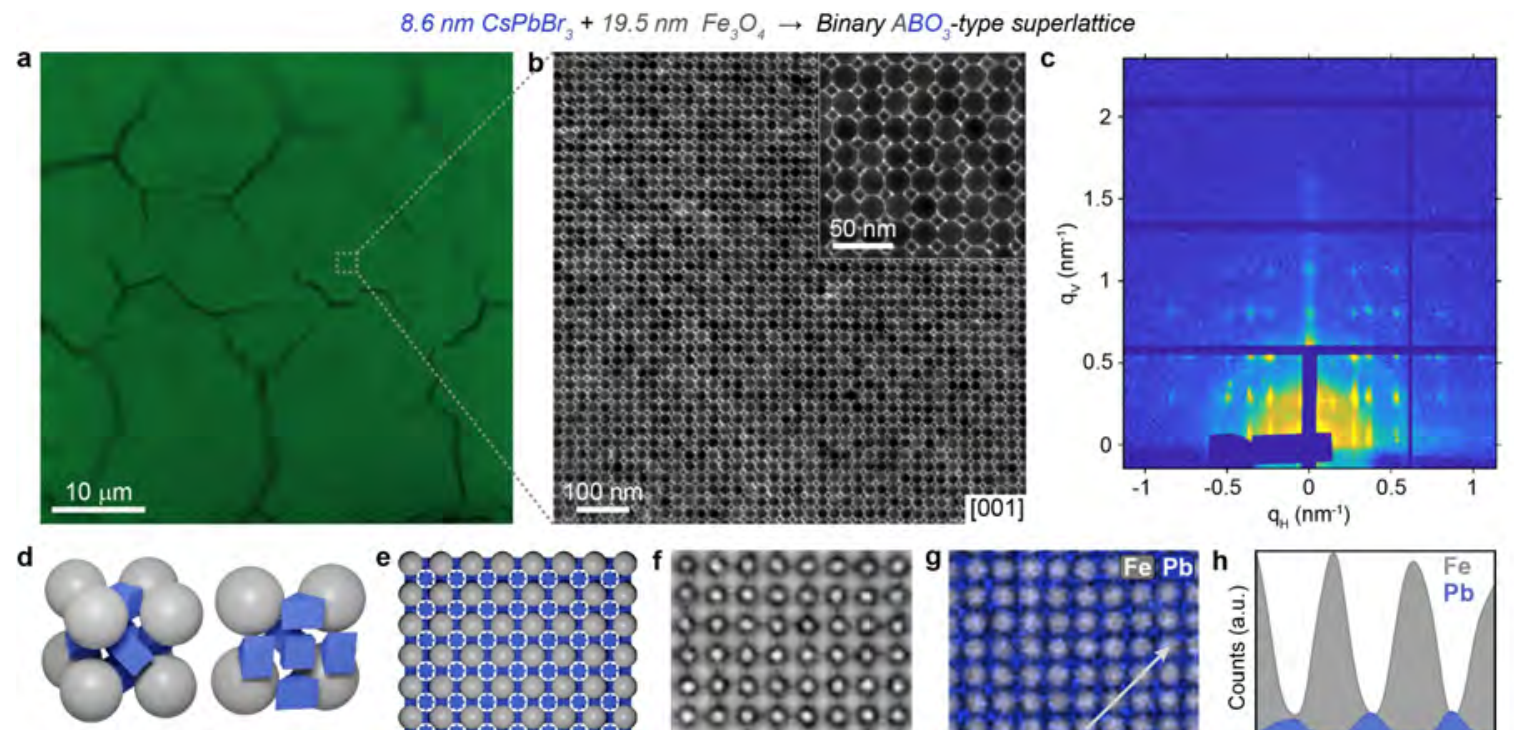

Binary $\mathrm{ABO}_{3}$-type $\mathrm{SL}$
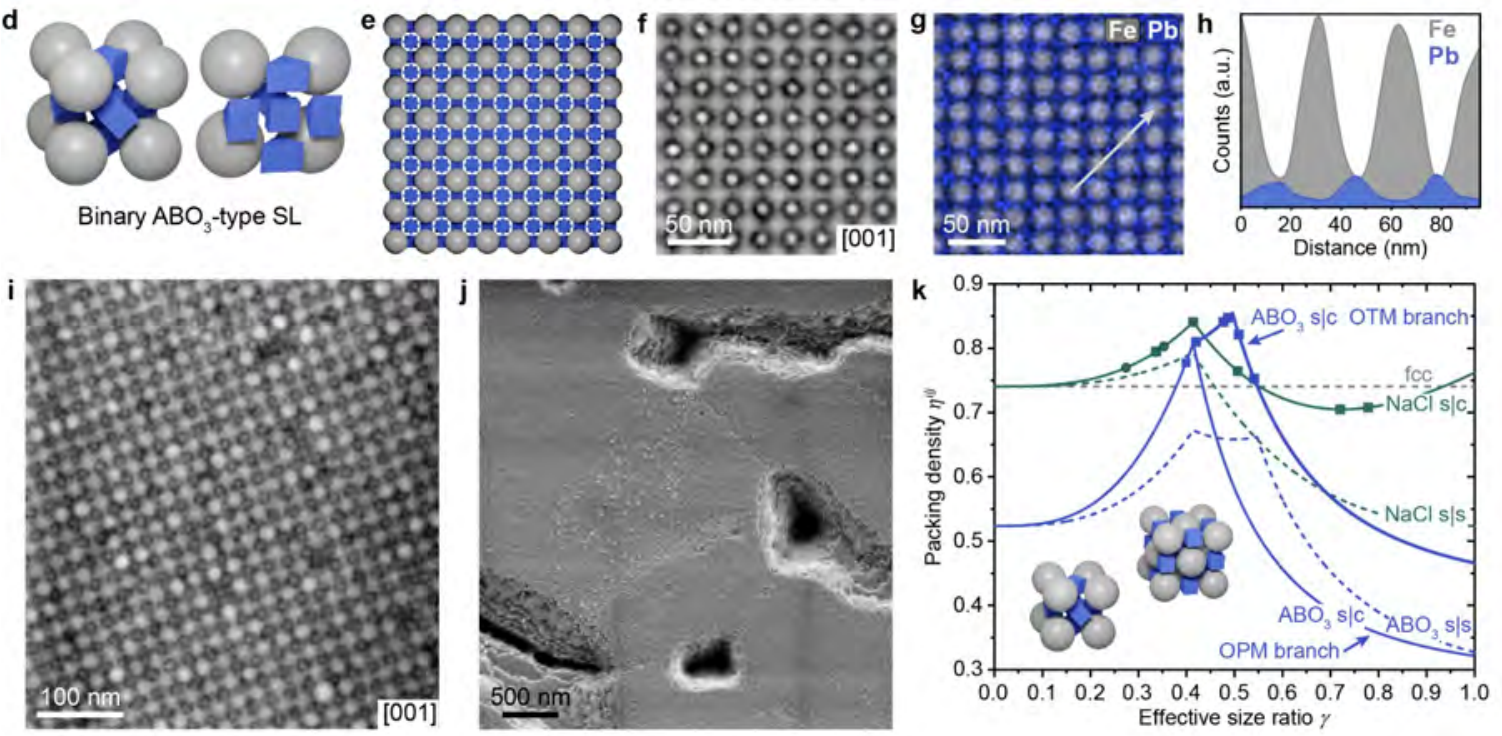

Fig. 1 | Characterization of a binary $\mathrm{ABO}_{3}$-type $\mathrm{SL}$ assembled from $8.6 \mathrm{~nm} \mathrm{CsPbBr}_{3}$ and $19.5 \mathrm{~nm} \mathrm{Fe}_{3} \mathrm{O}_{4} \mathrm{NCs}$ $(\boldsymbol{\gamma}=\mathbf{0 . 4 2 0})$. a, Optical microscope image under ultraviolet light. b, TEM images of a single $[001]_{\mathrm{SL}}$-oriented SL domain. c, 2D GISAXS pattern (on a $\mathrm{Si}_{3} \mathrm{~N}_{4}$-membrane), showing long-range in- and out-of-plane order in binary SL domains. d, $\mathrm{ABO}_{3}$ unit cell, with $\mathrm{Fe}_{3} \mathrm{O}_{4}$ shown as grey spheres and $\mathrm{CsPbr}_{3}$ - blue cubes. e, Crystallographic model of a [001 $]_{\mathrm{SL}}$-oriented $\mathrm{ABO}_{3}$ lattice. f, HAADF-STEM image of a [001 $]_{\mathrm{SL}}$-oriented domain. $\mathbf{g}$, EDX-STEM maps for $\mathrm{Fe}$ (grey, K-line) and $\mathrm{Pb}$ (blue, L-line) and (h) respective EDX line scans taken along the line shown in $\mathbf{g}$. $\mathbf{i}$, j, High-

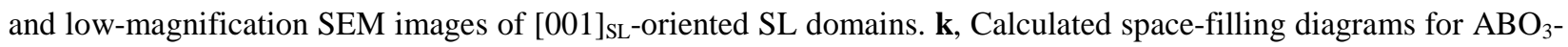
and NaCl-type binary SLs predicted by packing density analysis (OPM, except for indicated OTM-branch); the solid lines mark binary mixtures of larger spheres and smaller cubes $(\mathrm{s} \mid \mathrm{c})$, the dashed lines mark binary mixtures of different spheres (s|s). Experimentally observed $\mathrm{ABO}_{3}$ - and $\mathrm{NaCl}$-type lattices of this work are represented as dots at the respective $\gamma$-values (circles and rectangles denote SLs containing 5.3 and $8.6 \mathrm{~nm} \mathrm{CsPbBr} 3 \mathrm{NCs}$, respectively). The SL thickness is several hundred nm. 

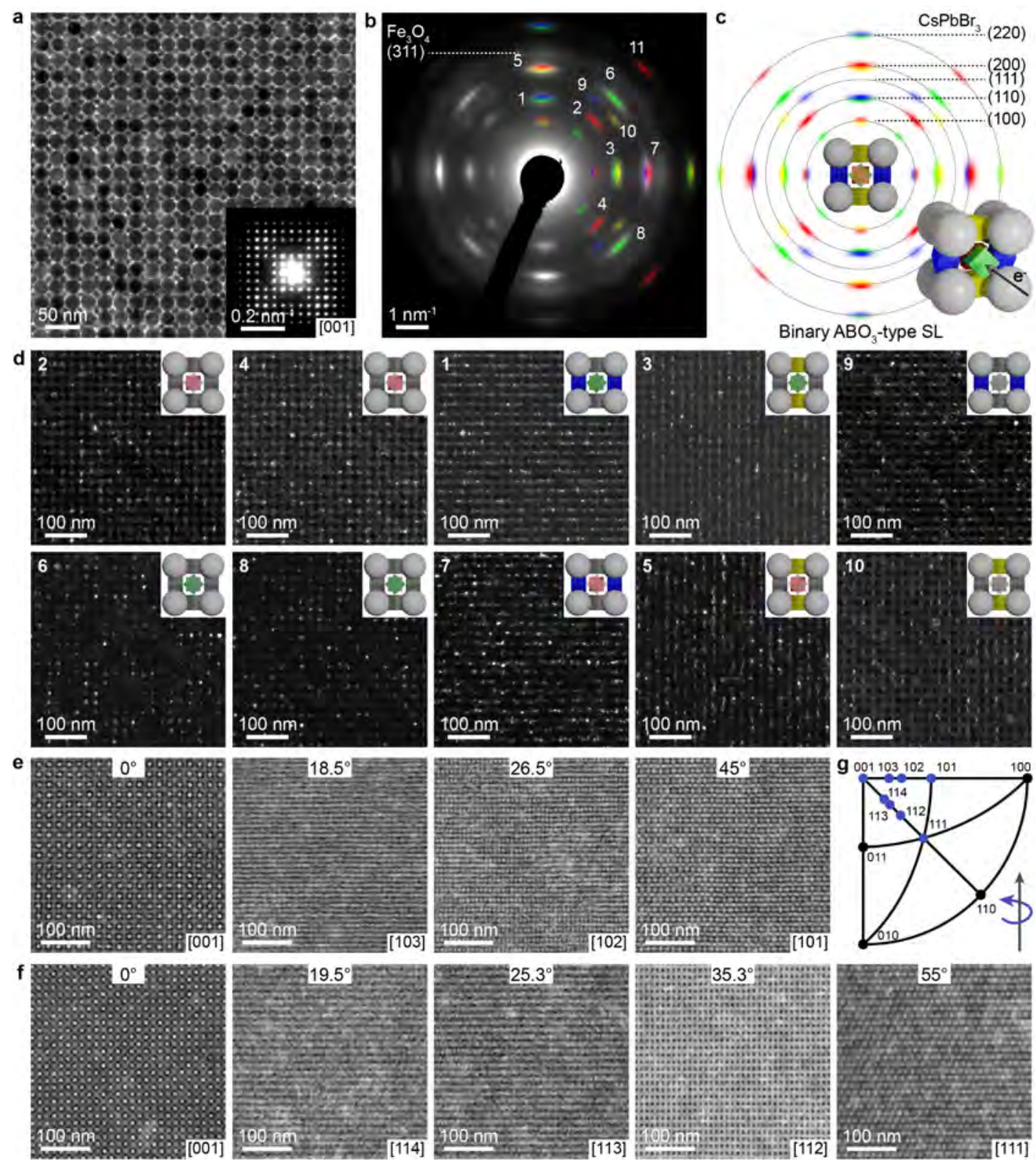

Fig. 2 | Structural characterization of binary ABO $_{3}$-type SLs. a, TEM image of [001 $]_{\mathrm{SL}}$-oriented domain assembled from $8.6 \mathrm{~nm} \mathrm{CsPbBr} 3$ and $19.5 \mathrm{~nm} \mathrm{Fe}_{3} \mathrm{O}_{4} \mathrm{NCs}$, along with the corresponding small-angle ED (inset) and (b) ED patterns. Owing to the small wavelength of electrons in TEM only lattice planes which are nearly parallel to the incident electron beam contribute to the diffraction pattern. Labelled diffraction arcs in $\mathbf{b}$ correspond to coloured $\mathrm{CsPbBr}_{3}$ nanocubes in c. c, Modeled ED pattern of [001 $]_{\mathrm{SL}}$-oriented $\mathrm{ABO}_{3}$-type binary SL $\left(\mathrm{Fe}_{3} \mathrm{O}_{4}\right.$ reflections are omitted for clarity). The colour of the reflections match the $\mathrm{CsPbBr}_{3}$ cubes they originate from. The red cube is located in the centre of the lattice and is rotated $45^{\circ}$ with respect to the six other cubes, which reside on faces having two $\mathrm{CsPbBr}_{3}$ $<110\rangle$ directions parallel to $\langle 100\rangle_{\text {sL. }}$ d, Dark-field TEM images for the individual kinds of ED reflections numbered in $\mathbf{b}$. Coloured cubes in the insets are those observed in the image. e, f, HAADF-STEM tilting series of the same binary SL domain comprising $14.5 \mathrm{~nm} \mathrm{Fe} \mathrm{O}_{4} \mathrm{NCs}$, obtained upon rotation around the [010] and [110] axes, respectively. g, Stereograph showing in blue the crystallographic projections of the $\mathrm{ABO}_{3}$ lattice accessed by tilting. 


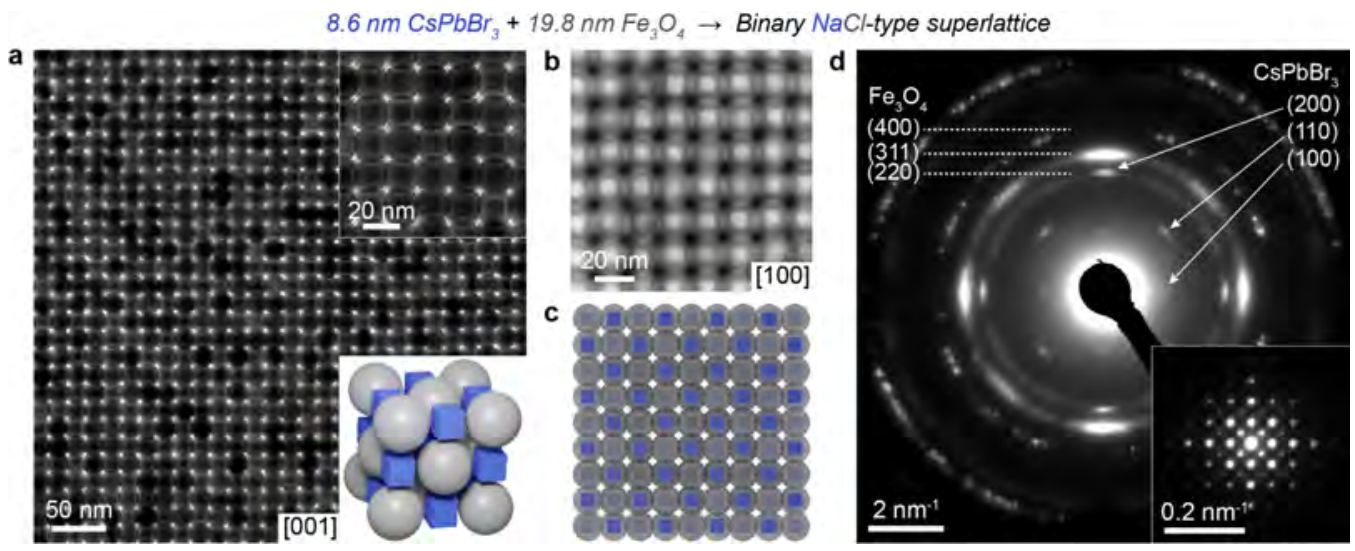

Fig. 3 | Characterization of NaCl-type binary SL formed from $8.6 \mathrm{~nm} \mathrm{CsPbBr} 3$ and $19.8 \mathrm{~nm} \mathrm{Fe}_{3} \mathrm{O}_{4} \mathrm{NCs}$ $(\gamma=\mathbf{0 . 4 1 4})$. a, TEM image of [001 $]_{\mathrm{SL}}$-oriented $\mathrm{NaCl}$-type SL domain. Top inset is higher magnification image, bottom inset shows $\mathrm{NaCl}$ unit cell with the preferable orientation of perovskite cubes. b, HAADF-STEM image of [001 sL $^{-}$ oriented domain. c, Crystallographic model of [001 $]_{\mathrm{SL}}$-oriented $\mathrm{NaCl}$ lattice (opacity of spheres is reduced for clarity). d, ED and (inset) small-angle ED pattern of a single domain shown in a. 


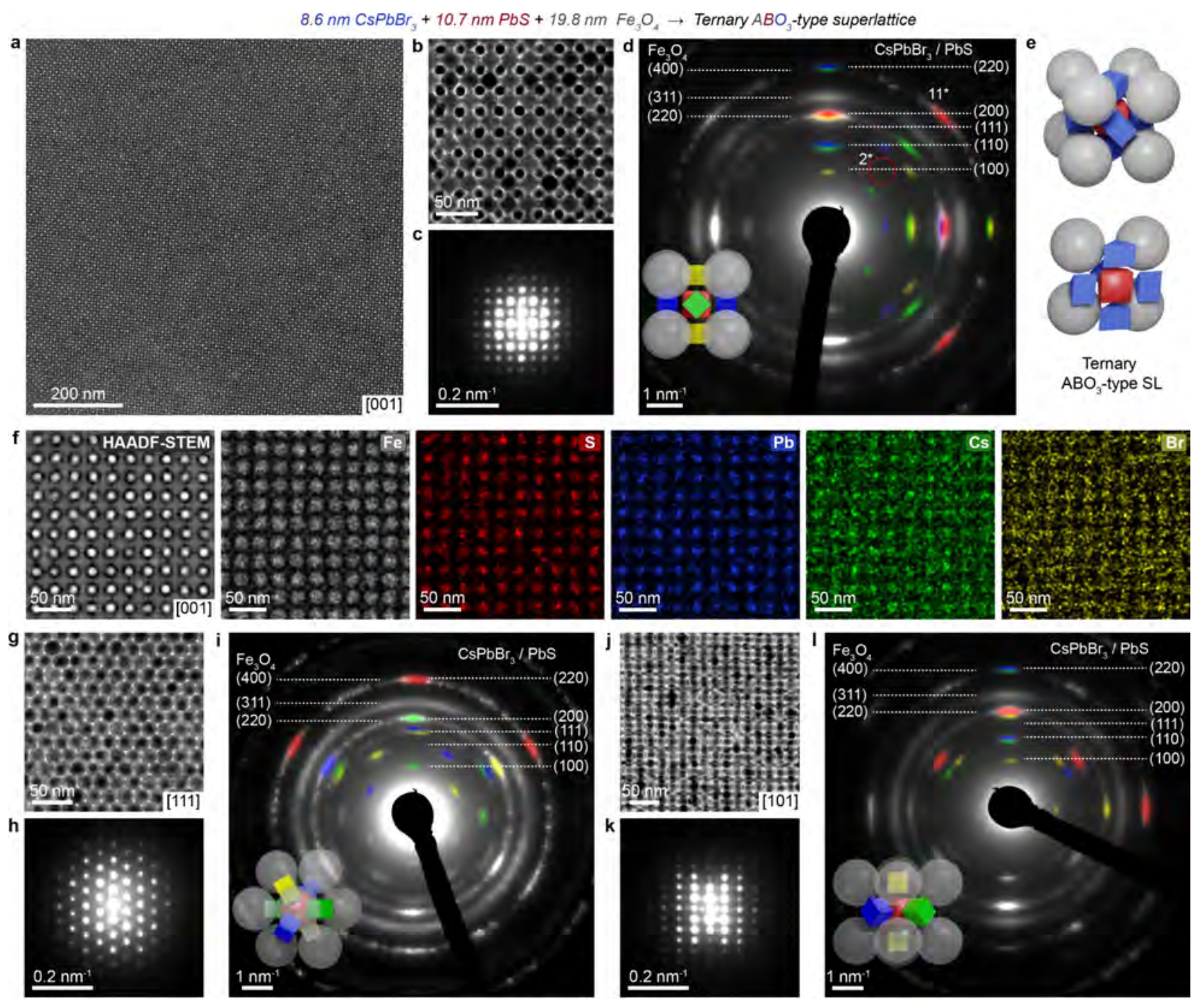

Fig. 4 | Characterization of ternary $\mathrm{ABO}_{3}$-type $\mathrm{SL}$ assembled from $8.6 \mathrm{~nm} \mathrm{CsPbBr}, 10.7 \mathrm{~nm} \mathrm{PbS}$ and $19.8 \mathrm{~nm}$ $\mathbf{F e}_{3} \mathbf{O}_{4}$ NCs. a, Large-area HAADF-STEM and (b) TEM images of single [001] $]_{\mathrm{SL}}$-oriented SL domains. c, small-angle ED and (d) ED patterns measured from the domain shown in $\mathbf{b}$. Simultaneous absence of an (110) arc expected from B-site $\mathrm{CsPbBr} 3$ cubes (marked as "2*", see Fig. 2b for binary SL) and high intensity of the (220) arc ("11*") point to exclusive occupation of the B-site by PbS NCs. e, $\mathrm{ABO}_{3}$ unit cell, with PbS shown as red bevelled cubes. f, HAADFSTEM image and corresponding EDX-STEM maps for Fe (grey, K-line), S (red, K-line), Pb (blue, L-line), Cs (green, L-line) and $\mathrm{Br}$ (yellow, K-line). g, j, TEM images, along with the corresponding (h, k) small-angle ED and (i, l) ED patterns of single domains in [111 $]_{\mathrm{sL}}$ and [101] sL projections, respectively. The origin of $\mathrm{CsPbBr}_{3}$ and $\mathrm{PbS}$ ED reflections in $\mathbf{d}, \mathbf{i}$ and $\mathbf{l}$ is colour-coded to match the models shown as insets. The appearance of (200) or (220) reflection accompanying the absence of (100) or (110) reflections in the relative directions indicates their origin from PbS NCs and the absence of identically oriented $\mathrm{CsPBr}_{3} \mathrm{NCs}$. 

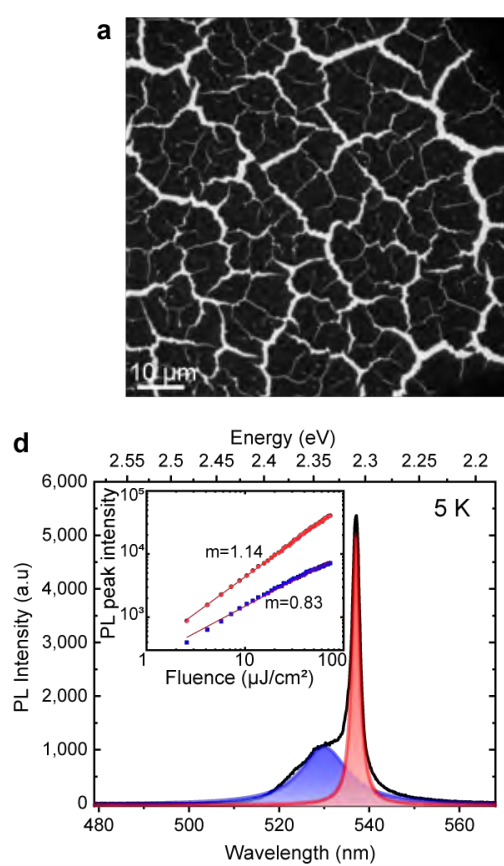

g

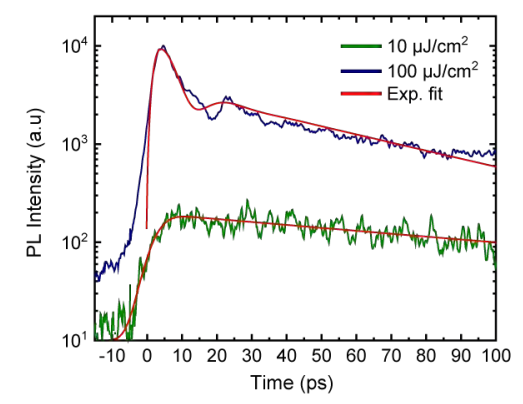

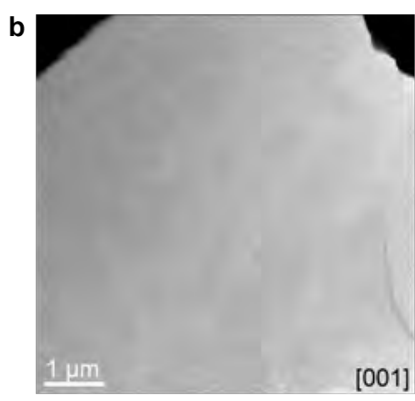
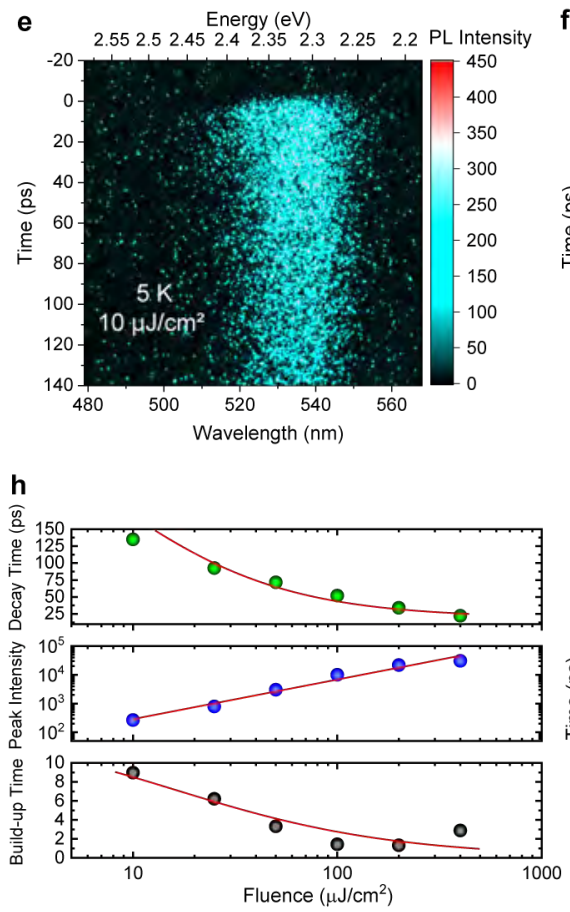
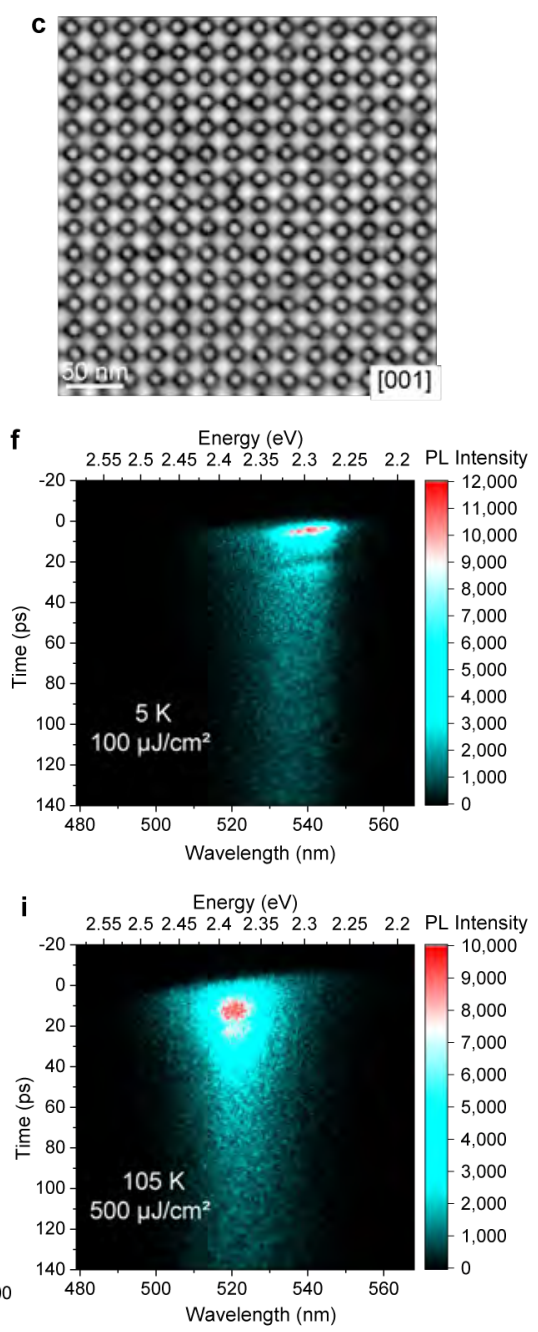

Fig. 5 | Superfluorescence from $\mathrm{ABO}_{3}$-type binary SLs from $8.6 \mathrm{~nm} \mathrm{CsPbBr} 3$ and $16.5 \mathrm{~nm} \mathrm{NaGdF} 4$. a, TEM and (b, c) HAADF-STEM images. d, Emission spectrum, comprising a higher-energy (uncoupled NCs) and a lower-energy band (coupled NCs). The shaded areas are fits to the data using Lorentzian functions, along with (as inset) corresponding integrated PL intensity (red and blue circles) plotted versus excitation fluence, where solid lines represent power-law fits with exponents $m$ of $c a$. 1. e, f, Streak camera images obtained with excitation fluences of $10 \mu \mathrm{J} / \mathrm{cm}^{2}$ and $100 \mu \mathrm{J} / \mathrm{cm}^{2}$, respectively. g, Spectrally-integrated time-resolved emission intensity traces for the two excitation fluences presented in $\mathbf{e}$ and $\mathbf{f}$. Red lines are weighted best-fits to a model that employs an exponential decay function and damped oscillations. $\mathbf{h}$, Top, Decay time as a function of the excitation fluence $\left(F_{e x}\right)$, fitted according to the SF model (red line): $\tau\left(F_{e x}\right)=\frac{\tau_{\text {uncoupled } N C}}{\zeta F_{e x}+1}+y_{0}$. Here, a fixed value of $\tau_{\text {uncoupled } N C}=350$ ps was used, as found for uncoupled $\mathrm{CsPbBr}_{3}$ NCs as well as for single NCs. Middle, PL peak intensity at a zero delay time, that increases superlinearly with excitation power, best fitted by a power-law with exponent 1.3 (red line). Bottom, the build-up time (in ps) decreases at high excitation fluences due to the increased interaction among the emitters. The red line is the best fit according the model described in the main text. i, Streak camera image obtained with excitation fluence of $500 \mu \mathrm{J} / \mathrm{cm}^{2}$ at $105 \mathrm{~K}$. 


\section{Methods.}

Synthesis of $\mathrm{CsPbBr}_{3}$ NCs. $8.6 \mathrm{~nm}$ were synthesized using oleylamine/oleic acid (OLA/OA) ligand system and then DDAB-treated as described in Ref. ${ }^{30}$.The OLA/OA-synthesis of $5.3 \mathrm{~nm} \mathrm{CsPbBr} 3 \mathrm{NCs}$ was adopted from Ref. ${ }^{32}$. The purified solution of $5.3 \mathrm{~nm} \mathrm{CsPbBr} 3$ NCs in toluene/hexane (2 ml, 50\%:50\% ratio, containing $9 \mathrm{mg} \mathrm{NCs}$ ) was stirred

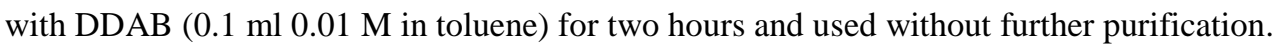

Synthesis of $\mathrm{Fe}_{3} \mathrm{O}_{4}$ NCs. Oleate-capped iron oxide NCs were synthesized by thermal decomposition of iron oleate complex in octadecene (ODE) according to Ref. ${ }^{52}$. The size of NCs was tuned from 9.6 to $25.1 \mathrm{~nm}$ by the OA concentration (from 0.12 to $0.95 \mathrm{M}$ ) and reaction temperature (from 308 to $319^{\circ} \mathrm{C}$ ). After three rounds of purification with hexane and acetone as a solvent/antisolvent pair, the NCs were dispersed in hexane.

Synthesis of $\mathbf{N a G d F}_{4} \mathbf{N C s}$. Hexagonal phase $\mathrm{NaGdF}_{4} \mathrm{NCs}$ were synthesized by thermal decomposition of gadolinium trifluoroacetate in the presence of sodium fluoride in OA/ODE ( 1 to 1 by volume) mixture at $312{ }^{\circ} \mathrm{C}$ for $1 \mathrm{~h}$, adopting reported procedure ${ }^{53}$. Larger NCs were obtained with lower Na-to-Gd ratios. After four washing steps with hexane and ethanol, the NCs were dispersed in hexane.

Synthesis of truncated cubic PbS NCs. 10.7 and $11.7 \mathrm{~nm}$ PbS NCs were synthesized by the reaction of lead oleate and sulfur at 200 and $205^{\circ} \mathrm{C}$, respectively, following reported procedure ${ }^{54}$. The NCs were washed four times with hexane and ethanol and dispersed in hexane.

Synthesis of $\mathbf{L a F}_{3}$ NCs. 9.2-26.5 nm (diameter) $\mathrm{LaF}_{3}$ nanodisks were synthesized by thermal decomposition of lanthanum trifluoroacetate in the presence of lithium fluoride in OA/ODE mixture (1:1 by volume) at $300{ }^{\circ} \mathrm{C}$ by the varying reaction time, similar to Ref. ${ }^{43}$. After three washing cycles with hexane and ethanol, the NCs were dispersed in hexane.

Preparation of multicomponent SLs. Self-assembly of NCs was carried out using a drying-mediated method on three kinds of substrates: carbon-coated TEM grids (carbon type B, Ted Pella, Formvar protective layer was removed by immerging the grid in toluene for $10 \mathrm{~s}$ ), silicon nitride membranes (TEMwindows) and HF-treated silicon. A mixture of NCs in anhydrous toluene had an overall particle concentration of $0.5-2 \mu \mathrm{M}$ and NC number ratios in the range of 0.5-20. 28-35 $\mu \mathrm{l}$ of NC mixture was transferred into a tilted $1.5 \mathrm{ml}$ glass vial with a substrate inside. The solvent was evaporated under $0.45 \mathrm{~atm}$ pressure at room temperature. For example, binary $\mathrm{ABO}_{3}-$, $\mathrm{AlB}_{2}$ - and $\mathrm{NaCl}$-type SLs with high yields were formed on TEM grids upon slow drying of a solutions prepared by mixing $19.8 \mathrm{~nm} \mathrm{Fe} \mathrm{O}_{4} \mathrm{NCs}$ $(40 \mathrm{mg} / \mathrm{ml}, 3.5 \mu \mathrm{l})$ and $8.6 \mathrm{~nm} \mathrm{CsPbBr} 3 \mathrm{NCs}(3.0 \mu \mathrm{M} ; 4.5 \mu \mathrm{l}, 2.4 \mu \mathrm{l}$ and $1.6 \mu \mathrm{l}$, respectively) with anhydrous toluene $(25 \mu \mathrm{l})$.

Electron microscopy characterization. TEM and HAADF-STEM images as well as ED and small-angle ED patterns were collected with the use of JEOL JEM2200FS microscope operating at $200 \mathrm{kV}$ accelerating voltage. Owing to the small wavelength of electrons (2.51 pm at $200 \mathrm{keV}$ ) the Bragg angles in ED are also small and, as a consequence, only lattice planes which are nearly parallel to the incident electron beam contribute to the diffraction pattern (electron beam is normal to the plane of the ED pattern). EDX-STEM maps and HAADF-STEM images at different tilt angle were recorded using an FEI Titan Themis microscope operated at $300 \mathrm{kV}$ equipped with a SuperEDX detector, with the aid of a motorized dual-axis tomography holder. Electron tomography was carried out in HAADF-STEM mode at $300 \mathrm{kV}$ using a small beam semi-convergence angle of $2.5 \mathrm{mrad}$, in order to increase the depth of field. Images were recorded over a tilt angle range from $-74^{\circ}$ to $+74^{\circ}$ in intervals of $2^{\circ}$. Reconstruction was done using the SIRT algorithm. The tomogram was recorded on a SL slightly inclined in respect to the electron beam, self-assembled on holey carbon coated TEM grid (Agar Scientific). TEM and electron diffraction images were compared with the ones simulated in Crystal Maker 10.4.5 and Single Crystal 3.1.5 software, purchased from CrystalMaker Software Ltd. SEM images were obtained on a FEI Helios 660 operated at $3 \mathrm{kV}$ using immersion mode.

GISAXS characterization. GISAXS measurements were performed at the Austrian SAXS beamline of the electron storage ring ELETTRA using a photon energy of $8 \mathrm{keV}^{55}$. The beamline setup was adjusted to a sample-to-detector distance of $1824 \mathrm{~mm}$ to result in an accessible horizontal $q$-range of $-1.2<q_{H}<1.8 \mathrm{~nm}^{-1}$ and vertical $q$-range of $0.1<q_{V}<2.9 \mathrm{~nm}^{-1}$. The X-ray beam was collimated to a spot-size at sample of approx. $200 \times 200 \mu \mathrm{m}$. All images 
were recorded using the Pilatus $1 \mathrm{M}$ detector (Dectris, Switzerland) with an exposure time of $10 \mathrm{~s}$ per image. Reference patterns to calibrate the $q$-scale were collected of silver-behenate ( $d$-spacings of $5.838 \mathrm{~nm})$. Samples were mounted on a 2-axis goniometer stage with $0.001^{\circ}$ angular precision, allowing us to ensure an incidence angle of $0.04^{\circ}$ for all measurements (determined by alignment of the specular reflection on the detector). All presented data was corrected for fluctuations of the primary intensity. Data treatment was done using the NIKA2D ${ }^{56}$ (geometry correction and calibration) as well as GIXSGUI ${ }^{57}$ (lattice indexing) software packages.

Optical properties of $\mathbf{C s P b B r}_{3}$ NCs. Optical absorption spectra were measured with Jasco V770 spectrometer in transmission mode. PL spectra were measured in a 90-degree configuration using Horiba Fluoromax-4P+ equipped with a photomultiplier tube and a monochromatized $150 \mathrm{~W}$ Xenon lamp as an excitation source. The PL quantum yield of the samples was measured in Hamamtsu Quantaurus-QY Plus UV-NIR absolute PL spectrometer (C13534-11) equipped with an integrating sphere. The SLs for PL quantum yield measurements were assembled on the square $5 \mathrm{~mm} \times 5 \mathrm{~mm}$ sapphire substrates.

Optical spectroscopy of SLs. For PL and time-resolved PL (Fig. 5 and Extended Data Figs. 9, 10), the sample was mounted in a Helium exchange-gas cryostat at $5 \mathrm{~K}$ (or higher temperature by using a heater when otherwise specified). A frequency-doubled regenerative amplifier seeded with a mode-locked Ti:sapphire laser with a pulse duration of 100$200 \mathrm{fs}$ and a repetition rate of $1 \mathrm{kHz}$ at $3.1 \mathrm{eV}$ photon energy was used as excitation source after passing through shortpass filters (442 nm cut-off wavelength). For both excitation and detection, we used the same focusing lens with $100 \mathrm{~mm}$ focal length, resulting in an excitation spot radius of about $60 \mu \mathrm{m}$. The recorded PL was long-pass filtered (480 nm cut-off wavelength) and then dispersed by a grating with 150 lines per $\mathrm{mm}$ in a $0.3 \mathrm{~m}$-long monochromator and detected with a streak camera with nominal time resolution of 2 ps and instrument response function FWHM of 4 ps. The time-integrated PL spectra were recorded by a $0.5 \mathrm{~m}$-long spectrograph with a grating with 300 lines per mm and a nitrogen-cooled CCD camera.

52. Park, J. et al. Ultra-large-scale syntheses of monodisperse nanocrystals. Nat. Mater. 3, 891-895 (2004).

53. Paik, T., Ko, D. K., Gordon, T. R., Doan-Nguyen, V. \& Murray, C. B. Studies of liquid crystalline selfassembly of $\mathrm{GdF}_{3}$ nanoplates by in-plane, out-of-plane SAXS. ACS Nano 5, 8322-8330 (2011).

54. Ibanez, M. et al. Electron doping in bottom-up engineered thermoelectric nanomaterials through HCl-mediated ligand displacement. J. Am. Chem. Soc. 137, 4046-4049 (2015).

55. Amenitsch, H., Bernstorff, S. \& Laggner, P. High-flux beamline for small-angle x-ray scattering at ELETTRA. Rev. Sci. Instrum. 66, 1624-1626 (1995).

56. Ilavsky, J. Nika: software for two-dimensional data reduction. J. Appl. Cryst. 45, 324-328 (2012).

57. Jiang, Z. GIXSGUI: a MATLAB toolbox for grazing-incidence X-ray scattering data visualization and reduction, and indexing of buried three-dimensional periodic nanostructured films. J. Appl. Cryst. 48, 917-926 (2015).

\section{Data availability}

The data that support the findings of this study are available from the corresponding author upon reasonable request. 
$8.6 \mathrm{~nm} \mathrm{CsPbBr}$ and $14.5 \mathrm{~nm} \mathrm{Fe}_{3} \mathrm{O}_{4}$ - Binary $\mathrm{ABO}_{3}$-type $\mathrm{SL}$
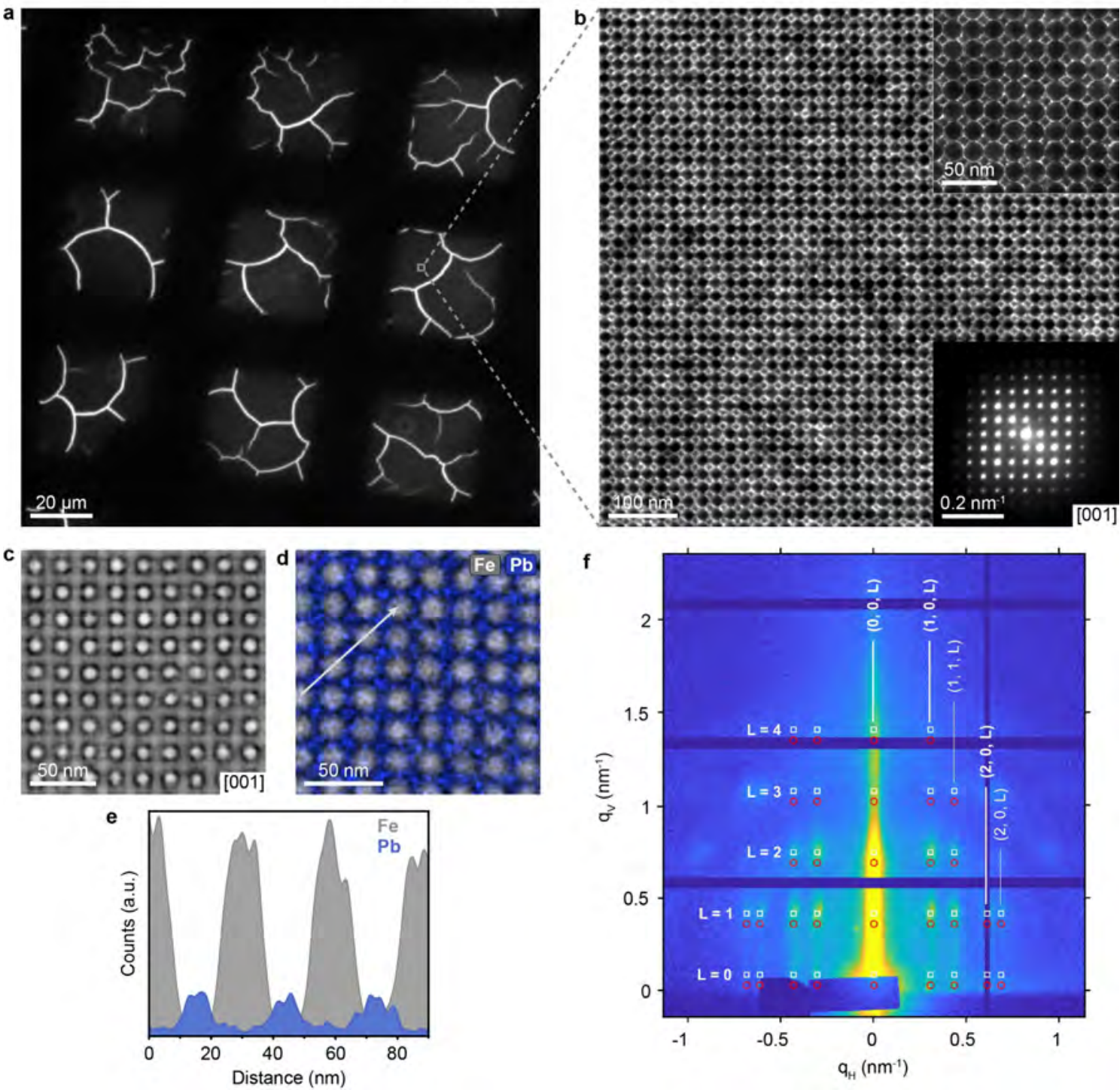

Extended Data Fig. 1 | Structural characterization of a binary $\mathrm{ABO}_{3}$-type $\mathrm{SL}$ comprising $8.6 \mathrm{~nm} \mathrm{CsPbBr}_{3}$ and $14.5 \mathrm{~nm} \mathrm{Fe}_{3} \mathrm{O}_{4}$ NCs $(\gamma=\mathbf{0 . 5 4 1})$. a, Low magnification TEM image showing large size of SL domains and homogeneous coverage over carbon-coated TEM grid. b. Zoomed-in TEM image from the area indicated by the box in a; upper inset presents higher-magnification TEM image; bottom inset is a small-angle ED pattern from a single SL domain in [001 $]_{S L}$ projection. c, HAADF-STEM image of a [001 $]_{\mathrm{SL}}$-oriented domain. d, EDX elemental maps of a $[001]_{S L}$-oriented domain for Fe (grey, K-line) and $\mathrm{Pb}$ (blue, L-line). e, EDX line scans taken along the line shown in d. f, 2D GISAXS pattern showing long-range order. The SL reflections can be indexed using a tetragonal $(P 4 / \mathrm{mmm})$ lattice: white markers correspond to the theoretical diffraction peak positions of an $a=b=20.5 \mathrm{~nm}$ and $c=19.0 \mathrm{~nm}$ unit cell (red markers show the corresponding Laue-type diffraction pattern). 


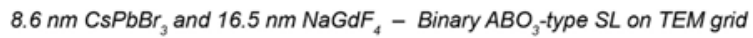
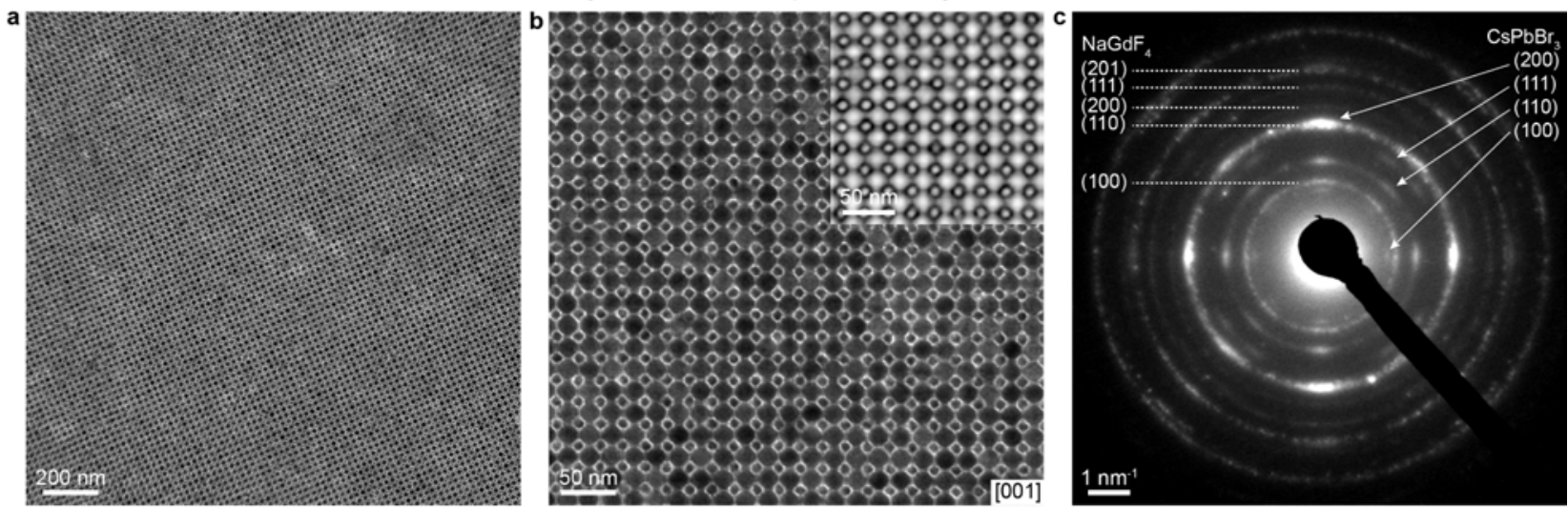

$8.6 \mathrm{~nm} \mathrm{CsPbBr} r_{3}$ and $16.5 \mathrm{~nm} \mathrm{NaGdF}_{4}-$ Binary $\mathrm{ABO}_{3}$-type $\mathrm{SL}$ on silicon substrate
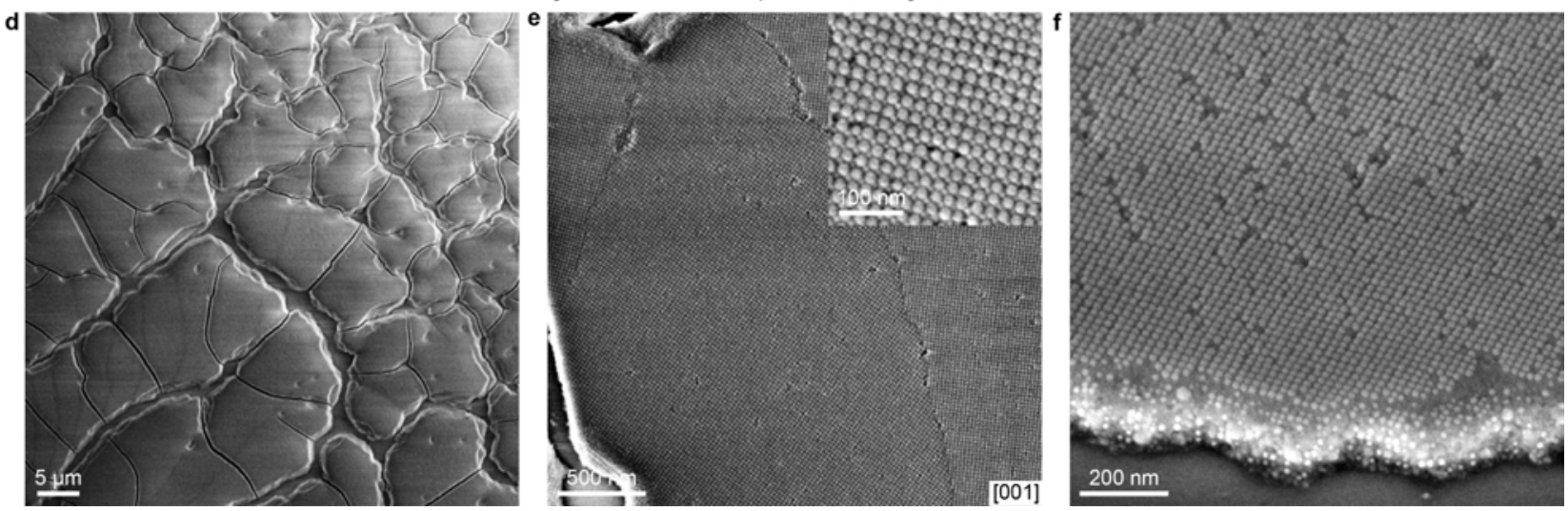

Extended Data Fig. 2 | Binary $\mathrm{ABO}_{3}$-type SL assembled from $8.6 \mathrm{~nm} \mathrm{CsPBr}_{3}$ and $16.5 \mathrm{~nm} \mathrm{NaGdF}$ NCs $(\gamma=\mathbf{0 . 4 8 6})$. a, b, TEM images at different magnification of a SL domain deposited on carbon-coated TEM grid, inset in $\mathbf{b}$ is HAADF-STEM image. c, ED pattern from an area displayed in $\mathbf{b}$. ED reflections from $\mathrm{CsPbBr}$ confirm $\mathrm{ABO}_{3}$ structure of the SL. d, e SEM images at different magnification showing large-area coverage of the silicon substrate by binary SL domains. f, Tilted SEM image revealing thickness of the SL domain. PL quantum yield of $\mathrm{ABO}_{3}$-type binary SL assembled from $8.6 \mathrm{~nm} \mathrm{CsPbBr} 3$ and $16.5 \mathrm{~nm} \mathrm{NaGdF} 4$ NCs on sapphire substrates is $\sim 50 \%$ and rises above $70 \%$ upon cooling with liquid nitrogen. 
$5.3 \mathrm{~nm} \mathrm{CsPbBr}$ and $12.5 \mathrm{~nm} \mathrm{Fe}_{3} \mathrm{O}_{4}-\mathrm{AlB}_{2}$-type $\mathrm{SL}$

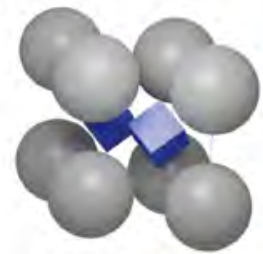

$\mathrm{AlB}_{2}$-type $\mathrm{SL}$
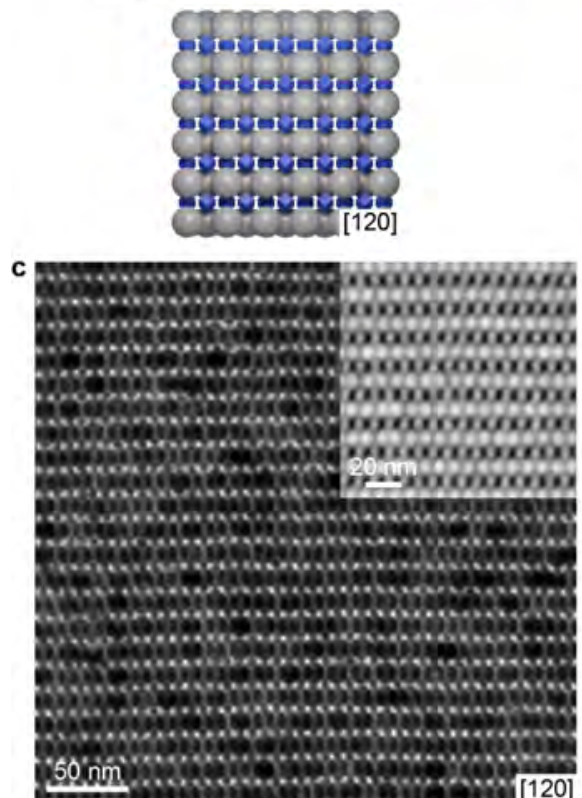

$8.6 \mathrm{~nm} \mathrm{CsPbBr}{ }_{3}$ and $19.8 \mathrm{~nm} \mathrm{Fe}_{3} \mathrm{O}_{4}-\mathrm{AlB}_{2}$-type SL
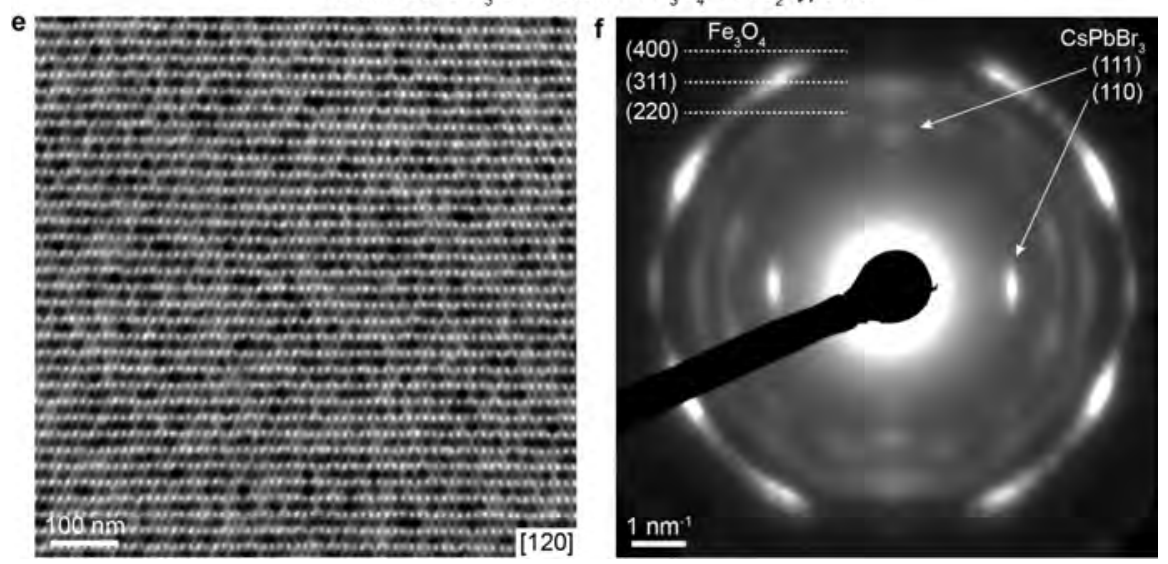

Extended Data Fig. 3 | AlB 2 -type binary SLs from (b-d) 5.3 nm CsPbBr 3 NCs combined with 12.5 nm Fe$_{3} \mathrm{O}_{4} \mathrm{NCs}$ $(\gamma=0.353)$ and $(e, f) 8.6 \mathrm{~nm}$ CsPbBr3 $\mathrm{NCs}$ combined with $\left.19.8 \mathrm{~nm} \mathrm{Fe} \mathrm{O}_{4} \mathrm{NCs}_{(} \boldsymbol{\gamma}=\mathbf{0 . 4 1 4}\right)$. a, AlB 2 unit cell, along with crystallographic models of [001]- and [120]-oriented $\mathrm{AlB}_{2}$ lattices. $\mathrm{Fe}_{3} \mathrm{O}_{4}$ shown as grey spheres and $\mathrm{CsPbBr} 3-$ blue cubes. b, TEM image of a SL domain in [001 $]_{\mathrm{SL}}$ orientation. c, e, TEM images of SL domains in [120] $\mathrm{SL}$ orientation, along with the corresponding $(\mathbf{d}, \mathbf{f})$ ED patterns; inset in c shows HAADF-STEM image. The presence of orthogonal reflections from (110) and (111) lattice planes of $\mathrm{CsPbr}_{3}$ indicates alignment of nanocubes within the SL such that [111] and [110] crystallographic directions of $\mathrm{CsPbBr}_{3}$ orient along [001] $]_{\mathrm{SL}}$ and [100] $]_{\mathrm{SL}}$, respectively. 


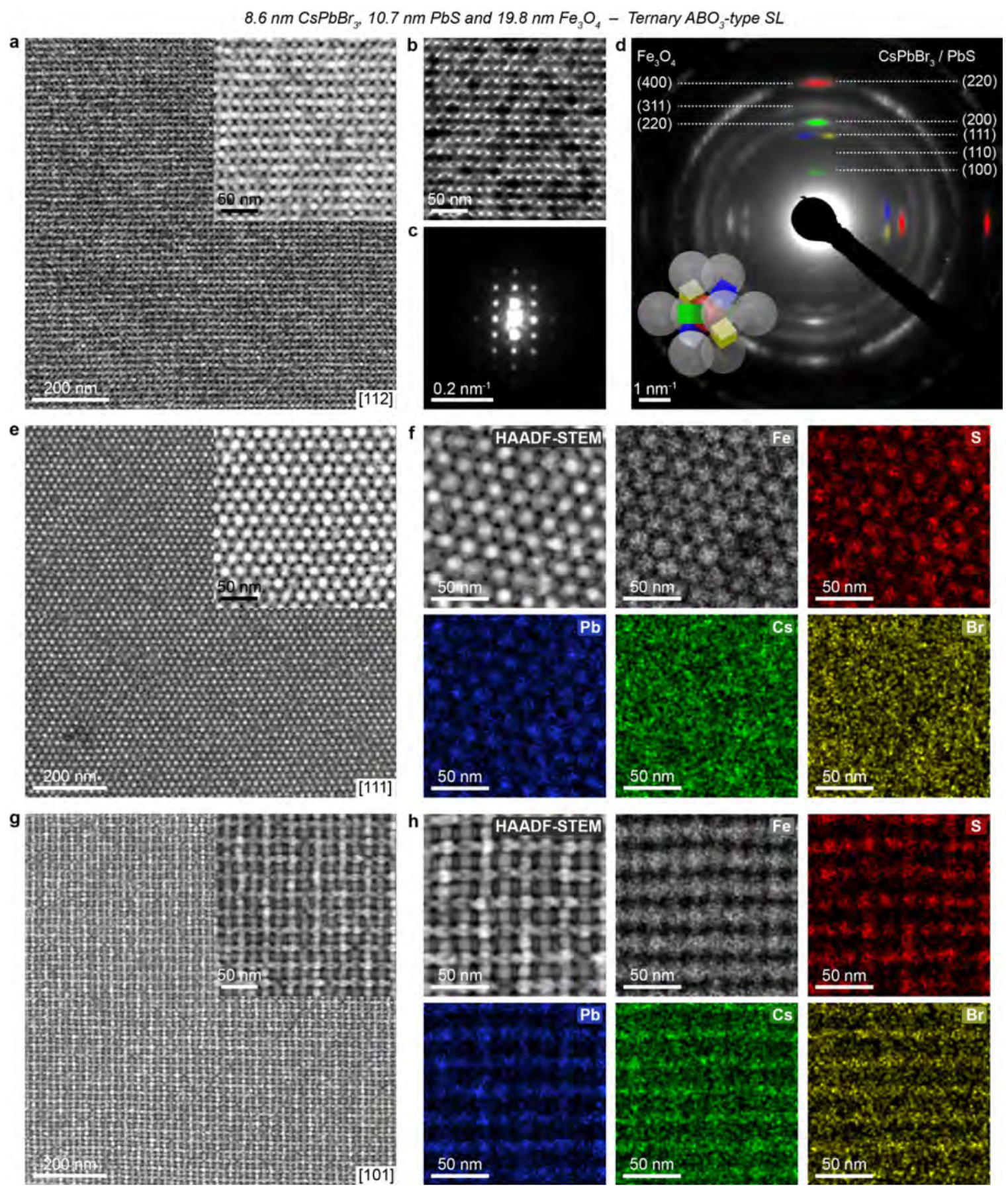

Extended Data Fig. 4 | Ternary $\mathrm{ABO}_{3}$-type SL domains with (a-d) [112]sL, (e, f) [111 $]_{\mathrm{SL}}$ and (g, h) [110]sL crystallographic orientations assembled from $8.6 \mathrm{~nm} \mathrm{CsPbBr} 3,10.7 \mathrm{~nm} \mathrm{PbS} \mathrm{NCs} \mathrm{and} 19.8 \mathrm{~nm} \mathrm{Fe} 3 \mathrm{O}_{4} \mathrm{NCs}_{\text {. a, e, }}$ g, HAADF-STEM images; insets show higher magnification images. b, TEM image of the [112 $]_{\mathrm{SL}}$-oriented domain, along with the corresponding (c) small-angle ED and (d) ED patterns; colour of diffraction arcs matches the NC orientations sketched as an inset (electron beam is normal to the plane of view). f, h, HAADF-STEM images and corresponding EDX-STEM maps for Fe (grey, K-line), S (red, K-line), $\mathrm{Pb}$ (blue, L-line), Cs (green, L-line) and $\mathrm{Br}$ (yellow, K-line). 

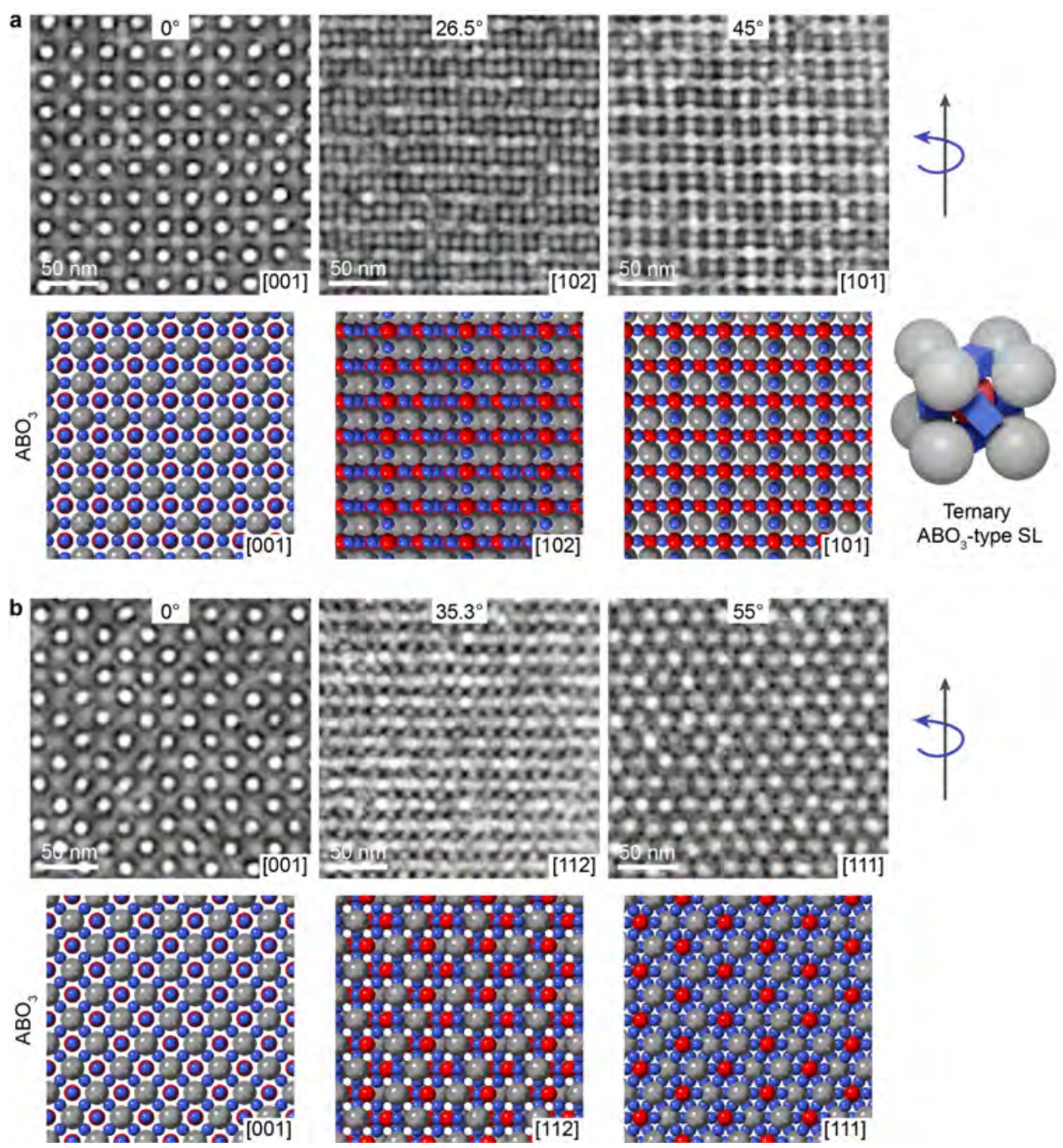

Extended Data Fig. 5 | HAADF-STEM tilting series around (a) [010] $]_{\mathrm{SL}}$ and (b) [110 $]_{\mathrm{sL}}$ axes of a ternary $\mathrm{ABO}_{3-}$ type SL comprising $8.6 \mathrm{~nm} \mathrm{CsPbBr} 3,10.7 \mathrm{~nm} \mathrm{PbS}$ and $19.8 \mathrm{~nm} \mathrm{Fe}_{3} \mathrm{O}_{4} \mathrm{NCs}_{3}$. HAADF-STEM images of a SL domain at different tilting angles match well the corresponding projections of the $\mathrm{CaTiO}_{3}$ structure. 


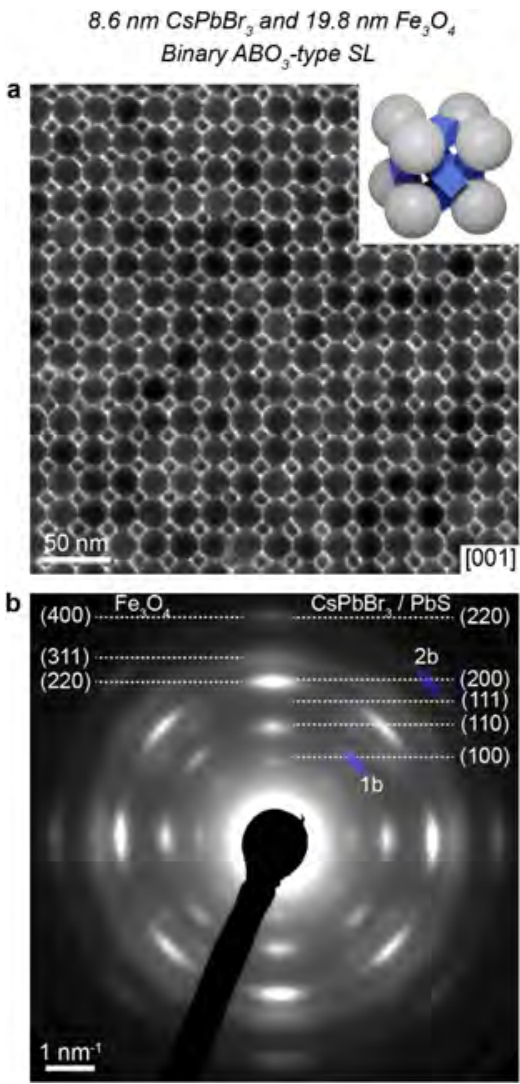

$8.6 \mathrm{~nm} \mathrm{CsPbBr}, 10.7 \mathrm{~nm} \mathrm{PbS}$ and $19.8 \mathrm{~nm} \mathrm{Fe}_{3} \mathrm{O}_{4}$ Partially ternary $\mathrm{ABO}_{3}$-type $S L$

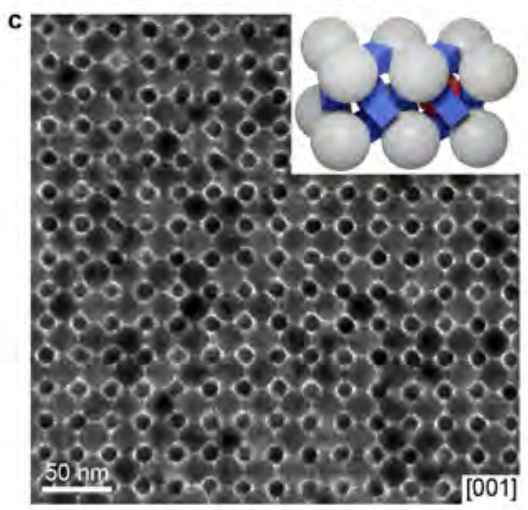

$8.6 \mathrm{~nm} \mathrm{CsPbBr}, 11.7 \mathrm{~nm} \mathrm{PbS}$ and $25.1 \mathrm{~nm} \mathrm{Fe}_{3} \mathrm{O}_{4}$
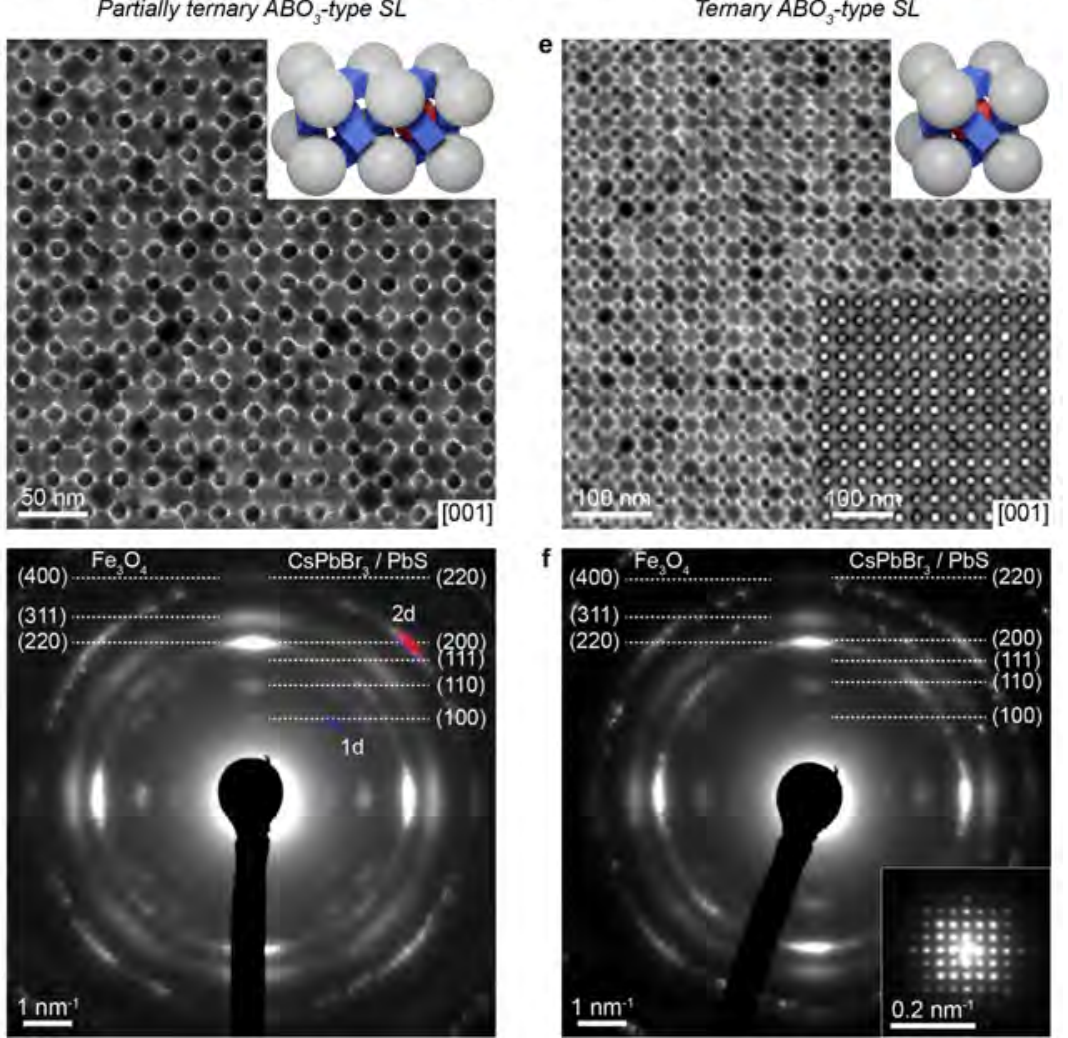

Extended Data Fig. 6 | Transition from binary $\mathrm{ABO}_{3} \mathrm{SL}$ comprising $8.6 \mathrm{~nm} \mathrm{CsPbBr} 3 \mathrm{NCs}$ and $19.8 \mathrm{~nm} \mathrm{Fe}_{3} \mathrm{O}_{4}$ NCs to ternary $\mathrm{ABO}_{3} \mathrm{SL}$ comprising $8.6 \mathrm{~nm} \mathrm{CsPbBr} 3 \mathrm{NCs}$ and $25.1 \mathrm{~nm} \mathrm{Fe}_{3} \mathrm{O}_{4}$ upon incorporation of 10.7$11.7 \mathrm{~nm}$ truncated cubic PbS NCs. a, TEM image and corresponding (b) ED pattern of a single binary [001 $]_{\mathrm{SL}^{-}}$ oriented domain assembled from $8.6 \mathrm{~nm} \mathrm{CsPbBr} 3$ and $19.8 \mathrm{~nm} \mathrm{Fe}_{3} \mathrm{O}_{4} \mathrm{NCs}$; inset in a: model of binary $\mathrm{ABO}_{3}$ lattice. c,

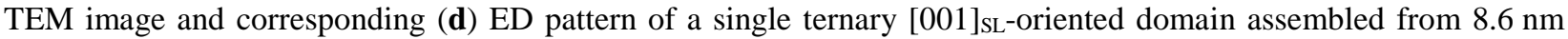
$\mathrm{CsPbBr}_{3} \mathrm{NCs}, 10.7 \mathrm{~nm} \mathrm{PbS}$ NCs and $19.8 \mathrm{~nm} \mathrm{Fe} \mathrm{O}_{4} \mathrm{NCs}$; inset in c: model of ternary $\mathrm{ABO}_{3}$ structure showing the formation of solid solution by substitution of $\mathrm{CsPBBr}_{3} \mathrm{NCs}$ on the B-site of the lattice by PbS NCs. The number ratio of $\mathrm{PbS}$ to $\mathrm{CsPbBr}_{3} \mathrm{NCs}$ in the mixture is too small to form exclusively ternary SL, as a result, both $\mathrm{CsPbBr}_{3}$ and $\mathrm{PbS}$ NCs are present on B-sites as is evident from ED patterns. In the ED of partially ternary lattice the intensity of (110) reflection "1d", which originates only from centre $\mathrm{CsPBBr}_{3}$, is weakened, compared to reflection "1b" in ED of binary SL, since the number of perovskite NCs on B-sites is reduced. Whereas the intensity of (220) reflection "2d" which originates from both $\mathrm{CsPbBr}_{3}$ and $\mathrm{PbS}$ NCs located on B-sites is enhanced compared to reflection "2b" in ED of binary $\mathrm{SL}$, as the scattering from $\mathrm{PbS} \mathrm{NCs}$, which contribute to this peak, is stronger than from $\mathrm{CsPbBr}_{3}$ lattice. As the degree of substitution increases, (111), (200), and (220) ED reflections for $\mathrm{CsPBr}_{3}$ and $\mathrm{PbS}$ NCs add up (because of similar lattice parameters) and give rise to higher intensity, while the (100) and (110) reflections, to which PbS NCs do not contribute due to their $F m \overline{3} m$ symmetry, eventually vanish (see also Fig. $4 \mathrm{~d}$ and Supplementary Figs. 5q, t). e, TEM

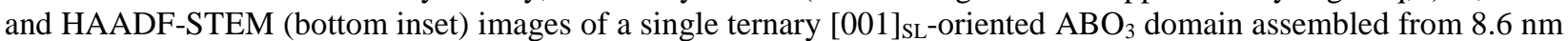
$\mathrm{CsPbr}_{3}, 11.7 \mathrm{~nm} \mathrm{PbS}$ and $25.1 \mathrm{~nm} \mathrm{Fe} \mathrm{O}_{4} \mathrm{NCs}$, along with the respective (f) ED and (inset) small-angle ED patterns; upper inset in e: model of ternary $\mathrm{ABO}_{3}$ lattice. $25.1 \mathrm{~nm} \mathrm{Fe} \mathrm{O}_{4} \mathrm{NCs}$ are too large to form binary $\mathrm{ABO}_{3}$-type SL, however the addition of $11.7 \mathrm{~nm}$ truncated cubic PbS NCs makes stable ternary $\mathrm{ABO}_{3}$-type SL. 


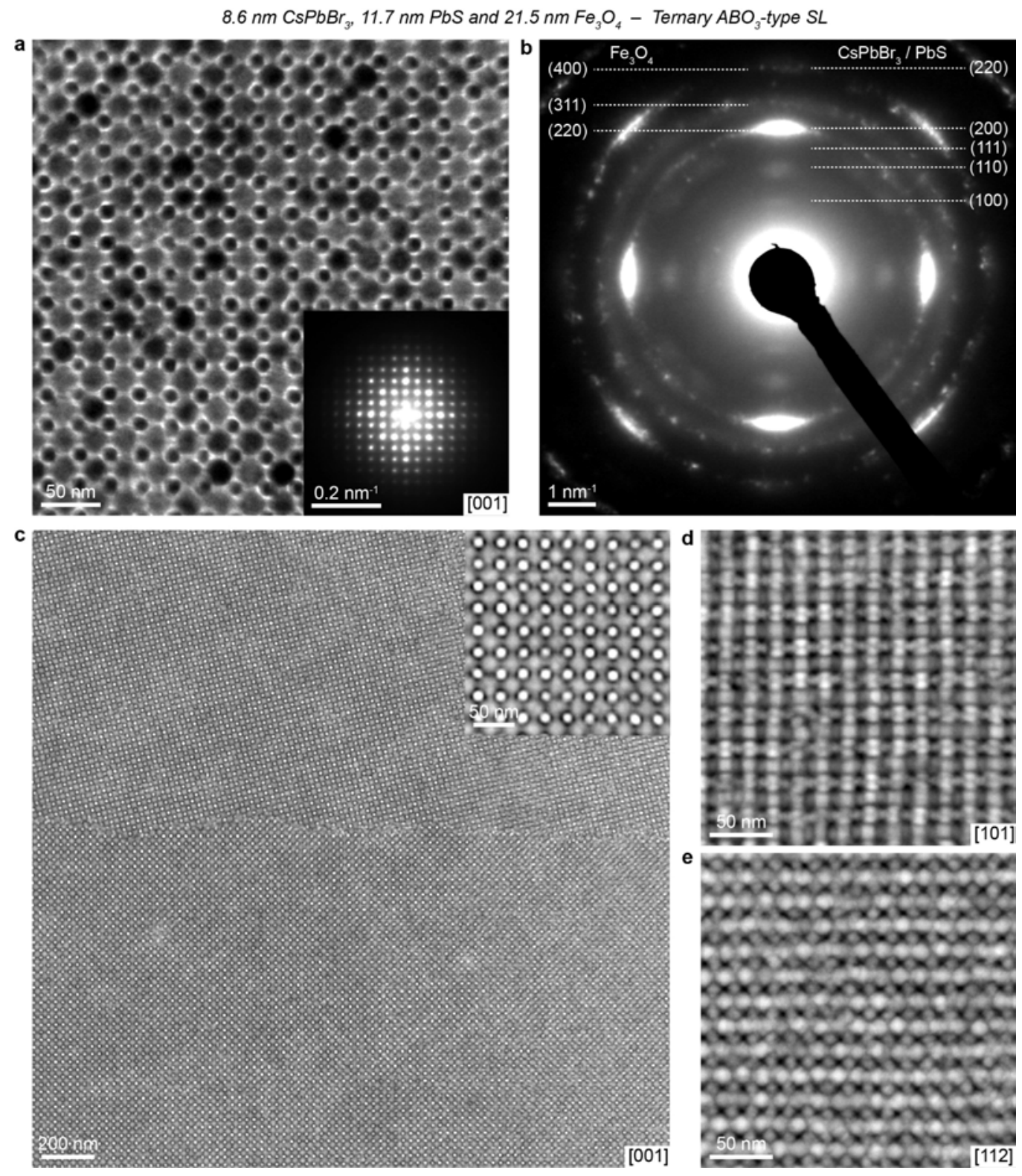

Extended Data Fig. 7 | Ternary $\mathrm{ABO}_{3}$-type SL domains assembled from 8.6 nm CsPbBr $3,11.7$ nm PbS NCs and $21.5 \mathrm{~nm} \mathrm{Fe} \mathrm{F}_{4}$ NCs. a, TEM image of a single SL domain in [001] $]_{\mathrm{SL}}$ orientation, together with the corresponding (inset) small-angle ED and (b) ED patterns. c, Low and high magnification HAADF-STEM images of [001 $]_{\mathrm{SL}}$-oriented

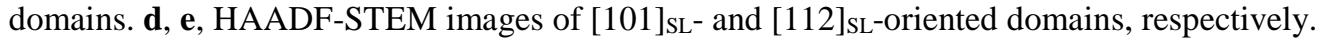



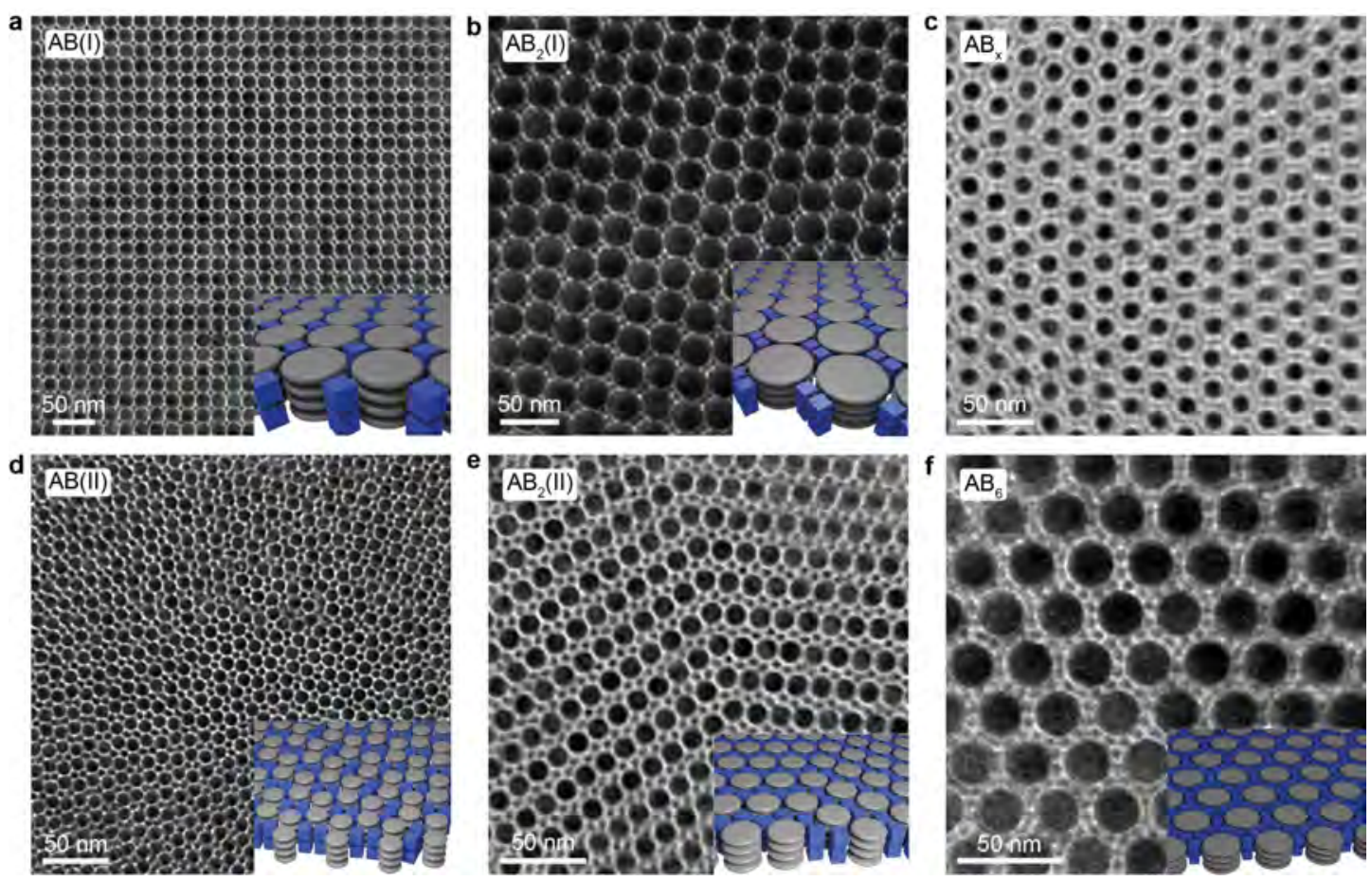

Extended Data Fig. 8 | TEM images and structural models of columnar binary SLs assembled from $5.3 \mathrm{~nm}$ CsPbBr 3 cubes and $\mathbf{L a F}_{3}$ nanodisks. a, $\mathrm{AB}(\mathrm{I})$-type $\mathrm{SL}$ comprising $16.6 \mathrm{~nm} \mathrm{LaF}_{3} \mathrm{NCs}$. b, $\mathrm{AB}_{2}(\mathrm{I})$-type $\mathrm{SL}$ comprising $26.5 \mathrm{~nm} \mathrm{LaF} 3$ NCs. c, $\mathrm{AB}_{\mathrm{x}}$-type SL comprising $12.5 \mathrm{~nm} \mathrm{LaF}_{3}$ NCs. d, AB(II)-type SL comprising $9.2 \mathrm{~nm} \mathrm{LaF}_{3} \mathrm{NCs}$. e, $\mathrm{AB}_{2}$ (II)-type SL comprising $12.5 \mathrm{~nm} \mathrm{LaF} \mathrm{NCs}_{3}$. f, $\mathrm{AB}_{6}$-type SL comprising $21.0 \mathrm{~nm} \mathrm{LaF} \mathrm{NCs}_{3}$. Six different columnar structures have been observed, as a result of adjusting the cube-to-disk size- and number-ratios. Interestingly, none of these structures had been reported for disks + spheres systems and also not observed by us, highlighting the crucial role that cubic shape plays for the formability of these structures (due to much higher resulting packing density compared to disks + spheres). The yield and the lateral extent of the SL grains are, however, considerably smaller than those of $\mathrm{ABO}_{3}$ - and $\mathrm{NaCl}$-type SLs and require further optimization. 

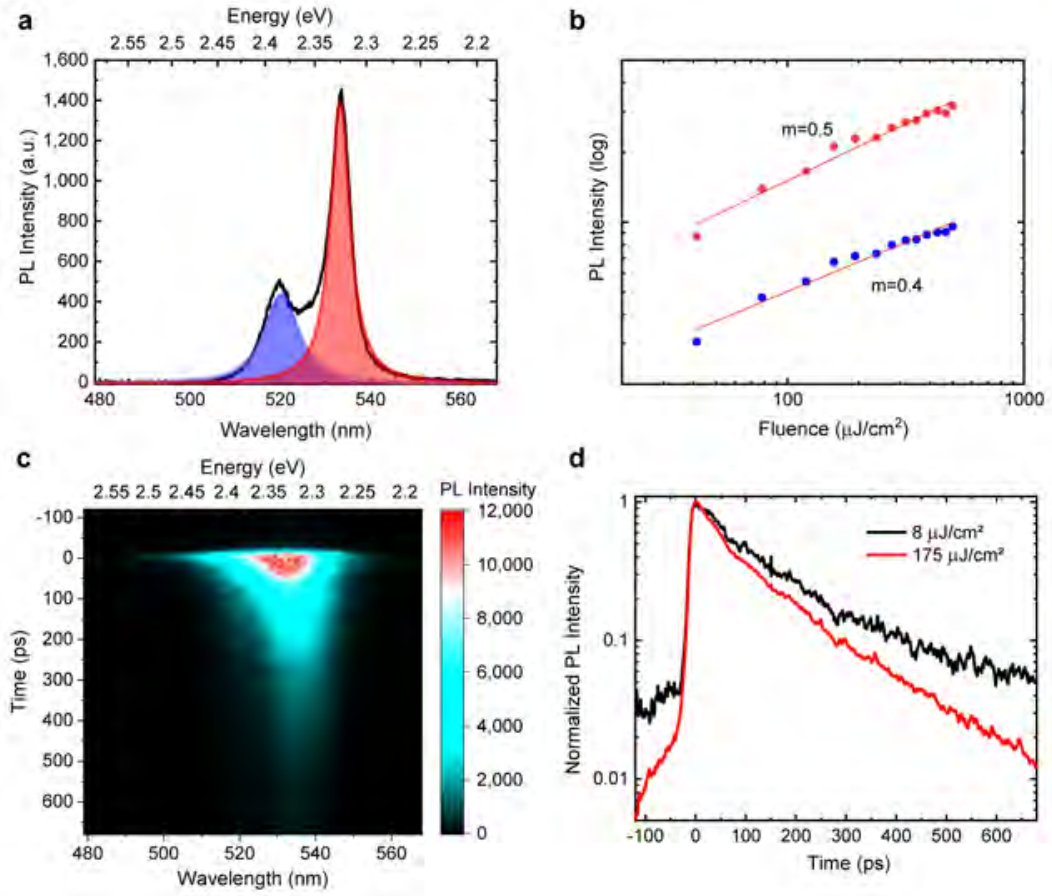

d

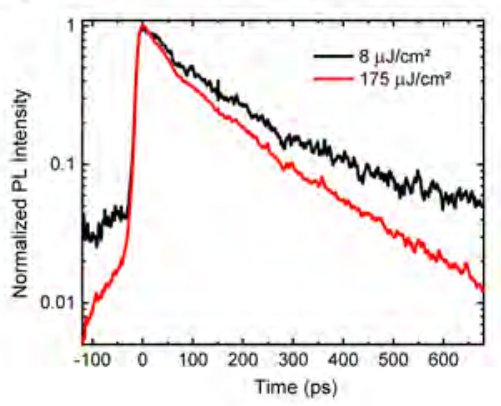

Extended Data Fig. 9 | Luminescence spectroscopy of $\mathrm{ABO}_{3}$-type binary SLs, made from $8.6 \mathrm{~nm} \mathrm{CsPBBr}_{3}$ and $16.5 \mathrm{~nm} \mathrm{NaGdF} 4$ NCs, on a carbon-coated Cu grid. a, PL spectrum where similarly to Fig. 5 of the Main Text, the PL spectrum is composed of two bands (coupled and uncoupled NCs). b. PL intensity for the uncoupled NCs (blue circles) and coupled NCs (red circles) bands, in a log-log plot. Fits to the data (red solid lines) reveal a sub-linear behaviour, with fitted power-law exponents $m$ of approximately 0.4-0.5, unlike to $\mathrm{Si}_{3} \mathrm{~N}_{4}$ as a substrate, attesting the occurrence of non-radiative processes at higher fluences, pointing to much enhanced SL-substrate interaction in the case of a conductive carbon film. c, Streak camera images obtained with an excitation fluence of $175 \mu \mathrm{J} / \mathrm{cm}^{2}$. Contrary to the results reported in the Main Text for $\mathrm{ABO}_{3}$-type $\mathrm{SLs}$ on $\mathrm{Si}_{3} \mathrm{~N}_{4}$-membranes, no evidence of drastic shortening or time oscillations could be found. Furthermore, a pronounced dynamic redshift characterizes the initial decay which could be related to thermal effects (rapid cooling after heating through the excitation pulse). This is in stark contrast with typical SF spectral dynamic, reported in Fig. 5 of the Main Text and in Ref. ${ }^{13}$, which exhibits a dynamic blueshift versus time ${ }^{13}$. d, Spectrally-integrated time-resolved emission intensity traces for two excitation fluences, $8 \mu \mathrm{J} / \mathrm{cm}^{2}$ and $175 \mu \mathrm{J} / \mathrm{cm}^{2}$ respectively. Although a slight shortening of the decay is clearly observed, this is probably due to a nonradiative process, presumably energy transfer to the substrate, given the sublinear fluence-dependence observed in $\mathbf{b}$ and reduction of fluorescence lifetime of uncoupled NCs from 350 ps to about 100 ps even at low fluences. Carboncoated grids might introduce absorbing states, which strongly influence the exciton dynamics and the onset of SF emission. This pronounced substrate effect is unsurprising given that SLs are morphologically two-dimensional, being $\leq 10$ unit cells in thickness. 

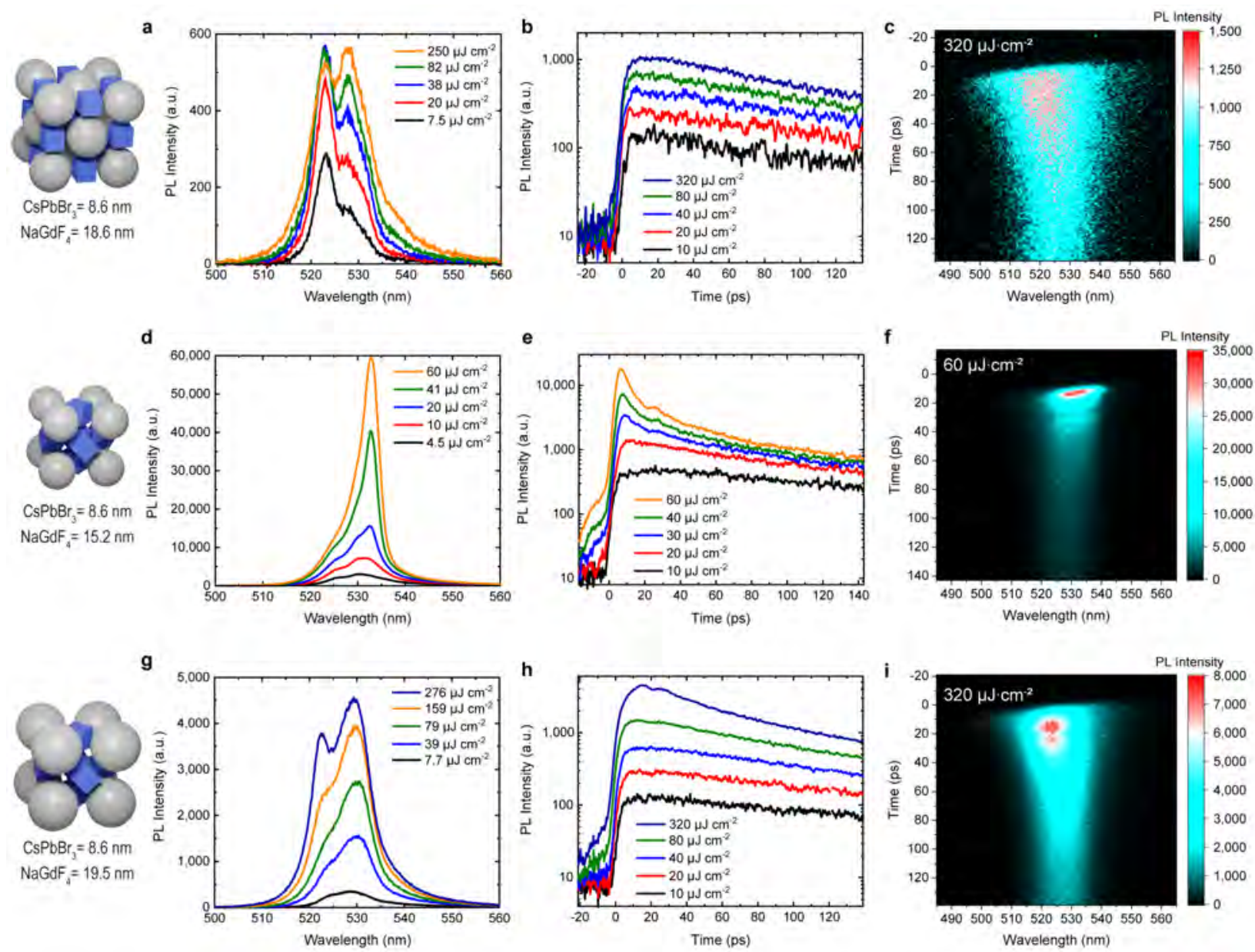

Extended Data Fig. 10 | Emission properties of different binary SLs comprising 8.6 nm perovskite NCs. Fluencedependent PL and time-resolved PL traces for (a, b) NaCl-type SLs employing $18.6 \mathrm{~nm} \mathrm{NaGdF} 4 \mathrm{NCs}$, (d, e) $\mathrm{ABO}_{3}-$ type SLs employing $15.2 \mathrm{~nm} \mathrm{NaGdF}_{4} \mathrm{NCs}_{\text {and }}\left(\mathbf{g}\right.$, h) $\mathrm{ABO}_{3}$-type SLs employing $19.5 \mathrm{~nm} \mathrm{NaGdF} 4 \mathrm{NCs}_{\text {. Typical streak }}$ camera images obtained at high fluences are reported in $\mathbf{c}, \mathbf{f}, \mathbf{i}$ for $\mathrm{NaCl}$ - and $\mathrm{ABO}_{3}$-type SLs, respectively. See Supplementary Note 4 for the discussion of results. 


\title{
Supplementary Information
}

\section{Perovskite-type superlattices from lead-halide perovskite nanocubes}

\author{
Ihor Cherniukh ${ }^{1,2}$, Gabriele Rainò ${ }^{1,2}$, Thilo Stöferle ${ }^{3}$, Max Burian $^{4}$, Alex Travesset $^{5}$, Denys \\ Naumenko $^{6}$, Heinz Amenitsch ${ }^{6}$, Rolf Erni ${ }^{7}$, Rainer F. Mahrt ${ }^{3}$, Maryna I. Bodnarchuk ${ }^{1,2}$, Maksym \\ V. Kovalenko ${ }^{1,2 *}$ \\ 1Department of Chemistry and Applied Bioscience, Institute of Inorganic Chemistry, ETH Zürich, Zürich, \\ Switzerland. \\ ${ }^{2}$ Laboratory of Thin Films and Photovoltaics, Empa - Swiss Federal Laboratories for Materials Science \\ and Technology, Dübendorf, Switzerland. \\ ${ }^{3}$ IBM Research Europe - Zürich, Rüschlikon, Switzerland. \\ ${ }^{4}$ Swiss Light Source, Paul Scherrer Institut, Villigen PSI, Switzerland. \\ ${ }^{5}$ Department of Physics and Astronomy, lowa State University and Ames Lab, Ames, lowa, USA. \\ ${ }^{6}$ Institute of Inorganic Chemistry, Graz University of Technology, Graz, Austria. \\ ${ }^{7}$ Electron Microscopy Center, Empa - Swiss Federal Laboratories for Materials Science and Technology, \\ Dübendorf, Switzerland
}

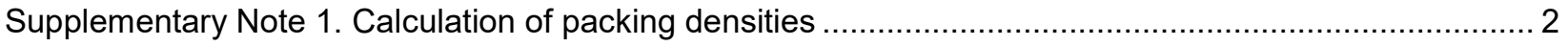

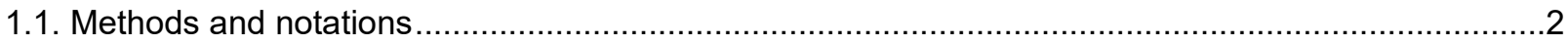

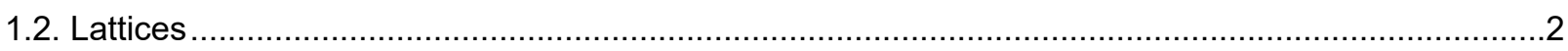

1.2.1. $\mathrm{NaCl}$

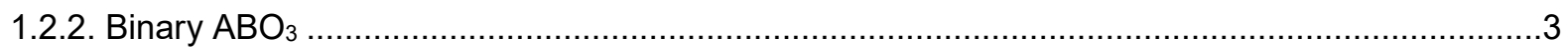

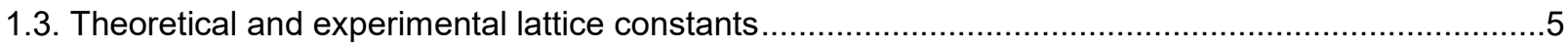

1.3.1. Lattice constants within hard sphere/cubes model ..............................................................

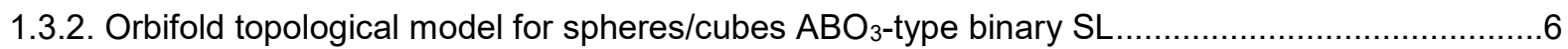

Supplementary Note 2. Relationship between crystallographic lattice planes and facets in $\mathrm{CsPbBr}_{3}$

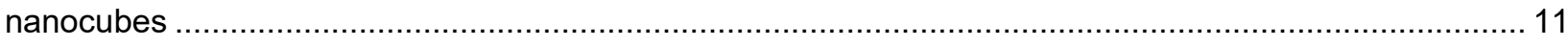

Supplementary Note 3. GISAXS characterization of SLs............................................................... 13

Supplementary Note 4. Superfluorescence in various binary superlattices .............................................. 15

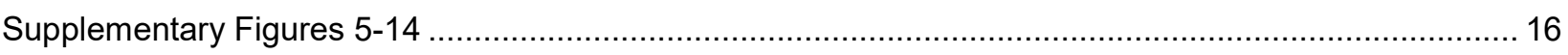

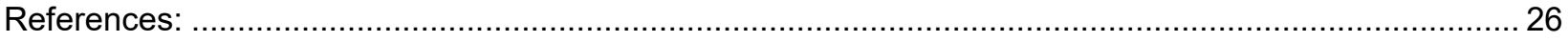




\section{Supplementary Note 1. Calculation of packing densities}

\subsection{Methods and notations}

We consider mixed systems of spheres of diameter $d_{s}=2 r_{s}$ and cubes of edge length $l_{c}$. The case where the spheres are the larger (A-particles, $d_{A}=d_{s}$ ) and the cubes are the smaller particles (B-particles, $\left.d_{B}=l_{c}\right) d_{s}>l_{c}$ is the main focus of this work. The packing fraction is the function of the effective size ratio, parameter $\gamma$, defined as

$$
\gamma=\frac{d_{B}}{d_{A}}=\frac{l_{c}}{d_{s}} \leq 1
$$

where $l_{c}$ is the edge length of the cube and $d_{s}$ is the diameter of sphere considered as hard cubes/spheres.

The general packing fraction will be denoted as

$$
\eta^{i \mid j}(\gamma)
$$

where $i, j-s$ or $c$. Thus, $\eta^{s \mid s}(\gamma)$ denotes the packing fraction of systems of hard spheres, $\eta^{s \mid c}(\gamma)$ - the packing fractions of spheres as A-particles and cubes as B-particles $(\gamma<1)$.

With cubes, there are additional degrees of freedom related to their orientation. The orientation of each cube will be denoted by the axis and angle of rotation ${ }^{58}$ :

$$
\text { Rotation parameters }=\{\vec{n}, \Phi\}
$$

The lattice constants and packing fractions are calculated by HOODLT ${ }^{59}$ as well as $\mathrm{HOOMD}^{60}$ using rigid constrains ${ }^{61}$ and the HPMC package ${ }^{62}$.

\subsection{Lattices}

\subsection{1. $\mathrm{NaCl}$}

From previous considerations ${ }^{63}$, the packing fraction for the $\mathrm{NaCl}$ lattice [space group $F m \overline{3} m(225)$, Wyckoff positions $2 a, 2 b]$ for an all-sphere case is

$$
\eta^{s \mid s}(\gamma)= \begin{cases}\frac{\sqrt{2} \pi}{6}\left(1+\gamma^{3}\right) & \gamma<\gamma_{c, 1} \\ \frac{2 \pi}{3} \frac{1+\gamma^{3}}{(1+\gamma)^{3}} & \gamma \geq \gamma_{c, 1}\end{cases}
$$

where $\gamma_{c, 1}=\sqrt{2}-1 \approx 0.4142$. The lattice constant is

$$
a_{L}(\gamma)=\left\{\begin{array}{ll}
\sqrt{2} d_{s} & \gamma<\gamma_{c, 1} \\
(1+\gamma) d_{s} & \gamma \geq \gamma_{c, 1}
\end{array} .\right.
$$

For B-particles cubes of edge $l_{c}=\gamma d_{s}$ oriented along the three primitive vectors, then the positions of the cube centers are the same as the corresponding inscribed spheres. Therefore, the only modification is that the packing fractions become

$$
\eta^{s \mid c}(\gamma)=\eta^{s \mid s}(\gamma) \frac{\pi+6 \gamma^{3}}{\pi\left(1+\gamma^{3}\right)}
$$

while the lattice constant is still given by Eq. S5. The lattice consists of each A touching 12 other $\mathrm{A}$ for $\gamma<\gamma_{c}$ and each $B$ with six $A$ contacts along the faces of the B-cubes for the other case. 


\subsubsection{Binary $\mathrm{ABO}_{3}$}

The $\mathrm{ABO}_{3}$ lattice is described by the space group $P m \overline{3} m(221)$ with a unit cell of one $\mathrm{A}$ particle in $1 \mathrm{a}$, another $\mathrm{B}$ in $1 \mathrm{~b}$ and three more $\mathrm{O}$ particles in positions $3 \mathrm{c}$.

All-sphere case. For spherical $B$ and $O$ particles, we consider them fully equivalent $(B=O)$. The packing fraction is

$$
\eta^{s s}(\gamma)=\left\{\begin{array}{cl}
\frac{\pi}{6}\left(1+4 \gamma^{3}\right) & \gamma<\gamma_{c, 1} \\
\frac{\pi \sqrt{2}}{3} \frac{1+4 \gamma^{3}}{(1+\gamma)^{3}} & \gamma_{c, 1} \leq \gamma<\gamma_{c, 2} \\
\frac{\pi}{48}\left(4+\frac{1}{\gamma^{3}}\right) & \gamma \geq \gamma_{c, 2}
\end{array}\right.
$$

with $\gamma_{c, 1}=\sqrt{2}-1 \approx 0.4142$ and $\gamma_{c, 2}=1 /(2 \sqrt{2}-1) \approx 0.5469$. For $\gamma<\gamma_{c, 1}$ each A particle has 6 A contacts, for $\gamma_{c, 1}$ $\leq \gamma<\gamma_{c, 2}$ each O particle has four contacts with an A particle, while A particles have 12, and finally, for $\gamma \geq \gamma_{c, 2}$, each $O$ particle has two contacts with each B particle and each B particle has 6 contacts with $O$ particles.

The lattice constant is given by

$$
a_{L}(\gamma)= \begin{cases}d_{s} & \gamma<\gamma_{c, 1} \\ \frac{1+\gamma}{\sqrt{2}} d_{s} & \gamma_{c, 1} \leq \gamma<\gamma_{c, 2} . \\ 2 \gamma d_{s} & \gamma \geq \gamma_{c, 2}\end{cases}
$$

Let us investigate the Orbifold topological model (OTM) branch for $\gamma>\gamma_{c, 1}$. It consists of four vortices in the $O$ particles, while the $A$ particles remain vortex-free so that the six A-A contacts remain. Therefore, it is

$$
\bar{\gamma}=\gamma_{c, 1} .
$$

This relation remains valid until the $\mathrm{B}$ and $\mathrm{O}$ particles contact each other, which occurs for $\gamma \equiv \bar{\gamma}_{c, 1}=0.5$, so that for $\bar{\gamma}_{c, 1} \leq \gamma<\gamma_{c, 2}$ it is

$$
\bar{\gamma}=2 \sqrt{2} \gamma-1 .
$$

Then, for $\gamma>\gamma_{c, 2}$ another OTM branch exists where

$$
\bar{\gamma}=\frac{1+(1-\sqrt{2}) \gamma}{\sqrt{2}}
$$

which applies until $O$ particles have contacts among each other at $\gamma=1$. Hence, the lattice constant of the OTM branch is given by

$$
a_{L, \text { OTM }}(\gamma)=\left\{\begin{array}{lc}
d_{s} & \gamma<\bar{\gamma}_{c, 1} \\
2 \gamma d_{s} & \bar{\gamma}_{c, 1} \leq \gamma<\gamma_{c, 2} \\
\frac{1+\gamma}{\sqrt{2}} d_{s} & \gamma \geq \gamma_{c, 2}
\end{array} .\right.
$$


The packing fraction is

$$
\eta_{O T M}^{s s}(\gamma)=\left\{\begin{array}{lc}
\frac{\pi}{6}\left(1+4 \gamma^{3}\right) & \gamma<\bar{\gamma}_{c, 1} \\
\frac{\pi}{48}\left(4+\frac{1}{\gamma^{3}}\right) & \bar{\gamma}_{c, 1} \leq \gamma<\gamma_{c, 2} . \\
\frac{\pi \sqrt{2}}{3} \frac{1+4 \gamma^{3}}{(1+\gamma)^{3}} & \gamma \geq \gamma_{c, 2}
\end{array}\right.
$$

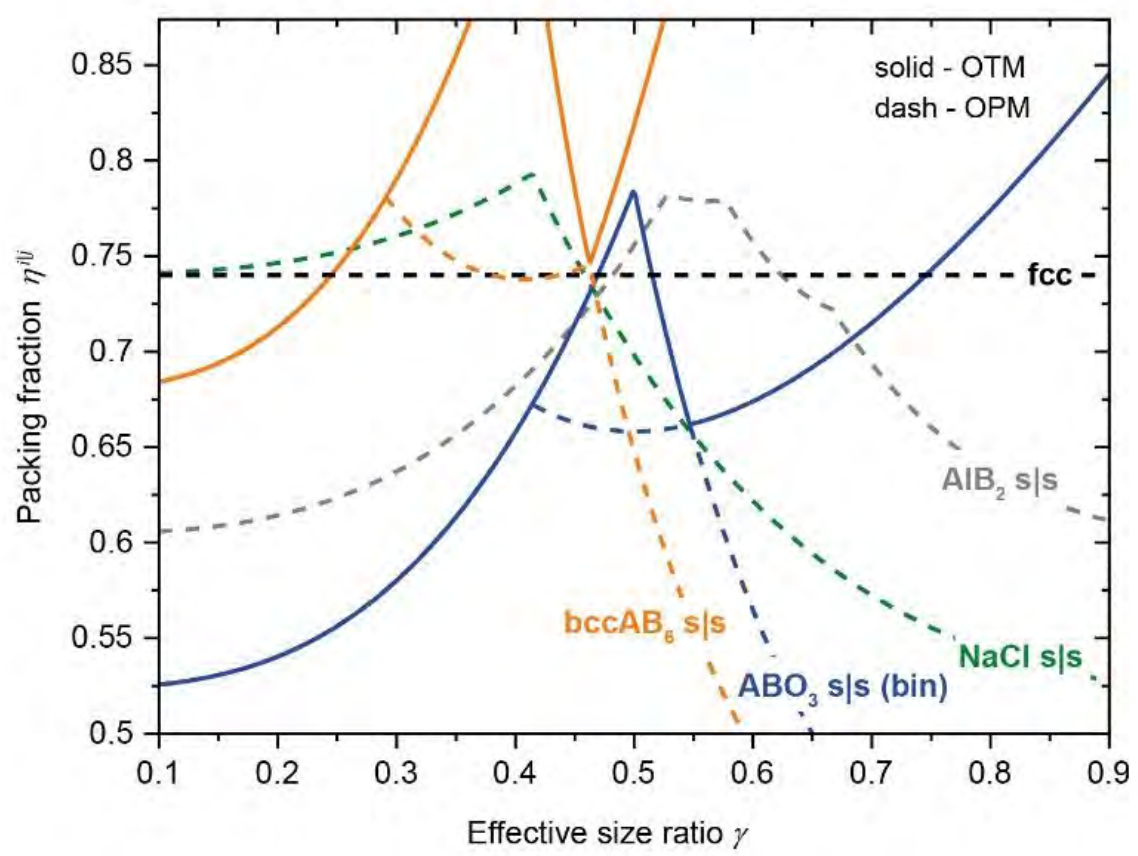

\section{Supplementary Fig. 1 | Packing fractions for the four different lattices considered, assembled from spherical particles.}

Note that at $\gamma=0.5, \mathrm{ABO}_{3}$-type lattice packing density $\left(\eta_{O T M}^{s s}(0.5) \approx 0.7854\right.$, Supplementary Fig. 1) may exceed that of two competing phases, $\mathrm{AlB}_{2}$ and $\mathrm{NaCl}$, but is lower than that of bccAB 6 (or $\mathrm{CaB}_{6}$ ) phases ${ }^{63}$, which are usually found experimentally ${ }^{64}$. Even after neglecting bccAB 6 , the formation of $\mathrm{ABO}_{3}$ would be expected only over a very narrow $\gamma$-range and, because this is only the OTM branch that has such high density, reaching these packing densities requires compression of the nanocrystal (NC) radius $r_{B}$ to $\bar{r}_{B}=2 \gamma_{c, 1} r_{B} \approx 0.8284 r_{B}$. This is a very significant compression that requires long ligands and/or small cores. In addition, such compression is difficult to realize for isotropic spherical NCs, unlike to cubes and other sharper shapes, which favour significant ligand bending in specific mutual geometries. On contrary, when switching to cubes as $\mathrm{B} / \mathrm{O}$-type NCs, the packing fraction for the $\mathrm{ABO}_{3}$ (within $\mathrm{OTM}$, as discussed below and shown in Fig. $1 \mathrm{k}$ of the Main Text) is high over a broader range of $\gamma$, and with compressions that are easily realizable. 
The case of cubes as B/O-particles. For B- and O-cubes of the same size, and in agreement with the observed electron diffraction, the positions and orientations (as described in Eq. S3) are given by

\begin{tabular}{c|c|c|c} 
Wyckoff & Coordinate & $\vec{n}$ & $\Phi$ \\
3c & $\left(\left(\frac{1}{2}, \frac{1}{2}, 0\right) a_{L}\right)$ & $(0,0,1)$ & $\frac{\pi}{4}$ \\
3c & $\left(\frac{1}{2}, 0, \frac{1}{2}\right) a_{L}$ & $(0,1,0)$ & $\frac{\pi}{4}$ \\
3c & $\left(0, \frac{1}{2}, \frac{1}{2}\right) a_{L}$ & $(1,0,0)$ & $\frac{\pi}{4}$ \\
1b & $\left(\frac{1}{2}, \frac{1}{2}, \frac{1}{2}\right) a_{L}$ & $(0,0,0)$ & 0
\end{tabular}

Then, for $\gamma<\gamma_{c, 1}$, the cubes are rattlers and the lattice constant is defined by the A-hard spheres. For $\gamma \geq \gamma_{c, 1}$, the optimal lattice constant corresponds to the $3 \mathrm{c}$ cubes having contacts at each of their vertices, with a packing fraction

$$
\eta^{s \mid c}(\gamma)= \begin{cases}\frac{\pi}{6}\left(1+\frac{24}{\pi} \gamma^{3}\right) & \gamma<\gamma_{c, 1} \\ \frac{\pi(5 \sqrt{2}-7)}{6}\left(\frac{24}{\pi}+\frac{1}{\gamma^{3}}\right) & \gamma \geq \gamma_{c, 1}\end{cases}
$$

with lattice constant

$$
a_{L}(\gamma)=\left\{\begin{array}{ll}
d_{s} & \gamma<\gamma_{c, 1} \\
\frac{\gamma}{\sqrt{2}-1} d_{s} & \gamma \geq \gamma_{c, 1}
\end{array} .\right.
$$

This solution consists of each A with 6 A contacts for $\gamma<\gamma_{c, 1}$ and each $3 c$ O-cube touching 8 other O-cubes at the vertices for $\gamma \geq \gamma_{c, 1}$.

\subsection{Theoretical and experimental lattice constants}

\subsubsection{Lattice constants within hard sphere/cubes model}

The hard-sphere diameter $d_{s}$ of a spherical $\mathrm{NC}^{63,65,66}$ is given by the optimal packing model (OPM) formula ${ }^{67}$

$$
\frac{d_{s}}{d_{\text {core }}} \equiv \tau=(1+3 \lambda \xi)^{1 / 3}
$$

in which $\lambda=\frac{2 L}{d_{\text {core }}}$ is the softness of the NC, where $L$ is the maximum stretched length of the ligand, and $\xi=\frac{\sigma}{\sigma_{\operatorname{Max}}}$, where $\sigma_{\operatorname{Max}}$ is the largest possible grafting density.

The hard-sphere effective diameter depends on the maximum grafting density $\sigma_{M a x}$, which depends on the core diameter ${ }^{66}$. For core diameters $d_{s}<5 \mathrm{~nm}$ these maximum grafting densities may be significantly enhanced by the curvature, but the NCs in the current experiment are much larger; therefore, we will use the large diameter value of $\sigma_{M a x} \approx 4.7 \mathrm{~nm}^{-2}$ for our calculations. The grafting densities of didodecyldimethylammonium (DDAB, $0.81 \mathrm{~nm}^{-2}$ for $\mathrm{CsPbBr}_{3} \mathrm{NCs}$ ) and oleic acid $\left(4.4 \mathrm{~nm}^{-2}\right.$ for $\mathrm{Fe}_{3} \mathrm{O}_{4}$ and 
$\mathrm{NaGdF}_{4}$ ) were derived from thermogravimetric analysis and from Ref. ${ }^{68}$ and were consistent with interparticle separation in single-component superlattices (SLs). The maximum extension length $(L=2.29 \mathrm{~nm})$ for oleic acid was calculated as described in Ref. ${ }^{63}$.

The grafting density for the cubes is relatively low. For example, under the assumption that DDAB may be represented as two hydrocarbon chains, then $\sigma_{\operatorname{Max}} \approx 4.7 / 2=2.35 \mathrm{~nm}^{-2}$. The hard cube is therefore defined by an edge $l_{c}$

$$
\left.l_{c}=l_{\text {core }}+2 \frac{\sigma}{\sigma_{\text {Max }}} L \approx l_{\text {core }}+1.12 \text { (in } \mathrm{nm}\right)
$$

where it has been assumed that the hydrocarbons are space-filling within their footprint area, that is, without any splay, which is the same assumption that leads to the OPM formula Eq. S17 defining the hard-sphere diameter for the spherical NCs. For the maximum extension length, we use the formula $L=0.122(n+1)$ (see Ref. ${ }^{63}$ for further discussion), with $n=13$ (there are 12 carbons on each side chain of DDAB, and 1 more carbon is included to account for the two additional methyl groups attached to the nitrogen).

The results for the softness parameters and hard-sphere effective diameters $\boldsymbol{d} s$ are shown in Supplementary Table 1 for all NC samples used in this work, along with hard-cube edge length $\left(\boldsymbol{I}_{c}\right)$ for cubes. One can then compute the parameter $\gamma$, defined in Eq. S1 and shown in Supplementary Table 2 for each binary SL. Then, the lattice constants are calculated from the formulas (Eq. S5 and S16) described above and, in Supplementary Table 2, are compared to the experimental lattice parameters derived from TEM images and GISAXS measurements.

One should note that the theoretical results are pure predictions that do not include any fitting parameters. The discrepancy with experiments is at most of a few $\AA$, which, given the uncertainties in terms of core radius or even lattice constant (when not measured by X-ray), is an excellent agreement. The only exception occurs for the $\mathrm{ABO}_{3} \mathrm{SL}$ for $\gamma>\gamma_{\mathrm{c}, 1}$. As is evident from the Main Text Fig. 1k, these values of $\gamma$ correspond to low < 0.5 packing fractions, which indicates that some of the NCs are not accurately represented by hard spheres or cubes and require the considerations of ligand textures ${ }^{63,65}$, i.e. "vortices", as discussed below with the OTM model.

\subsubsection{Orbifold topological model for spheres/cubes $\mathrm{ABO}_{3}$-type binary $\mathrm{SL}$}

The calculation of the hard-cube edge Eq. S18 assumes that ligands form a maximally dense flat brush, so it becomes exact for very large cubes, namely, when $l_{\text {core }}>\frac{\sigma}{\sigma_{M a x}} L \approx 1$. Even if these conditions are not strictly satisfied, Eq. S18 remains a good approximation if the lattice constant is determined by cubes interacting through their faces or with spherical NCs. However, in the $\mathrm{ABO}_{3}$ lattice for $\gamma \geq \gamma_{c, 1}$, see Eq. S7, the cubes interact through their vertices, and therefore, there is the possibility of ligand textures that bend away from the cube faces, enabling a denser packing, similarly as reported for spherical NCs whenever vortices $^{63,65}$ are allowed (see Supplementary Fig. 2).

Because the ligand texture deformations are along the corners, we define a modified effective size ratio

$$
\bar{\gamma}=\frac{\bar{l}_{c}}{d_{s}}
$$

where $\bar{l}_{c}<l_{c}$ is the deformed cube edge as determined by the cube corners. The lattice constant is given by Eq. S16, hence

$$
a_{L, O T M}(\bar{\gamma})=\frac{\bar{\gamma}}{\sqrt{2}-1} d_{s}
$$



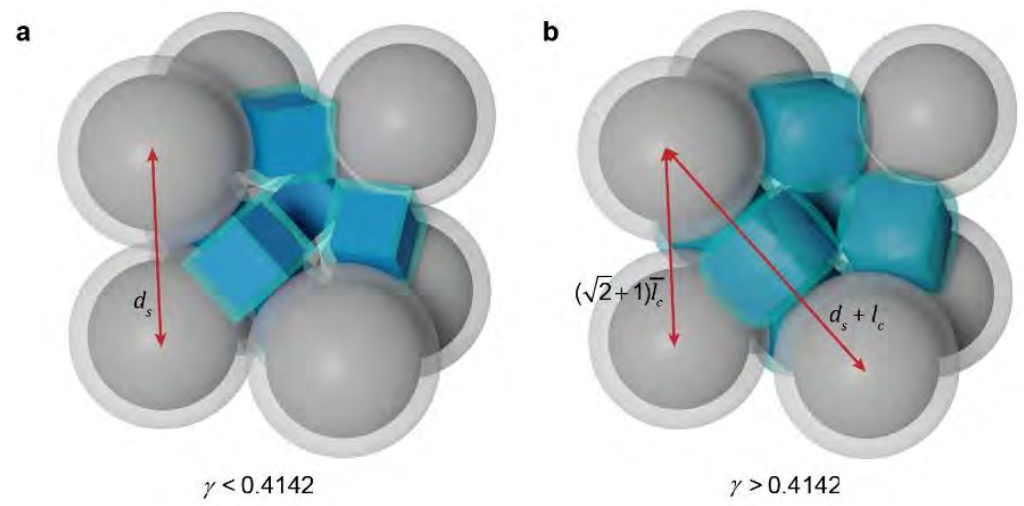

$$
\gamma<0.4142 \quad \gamma>0.4142
$$

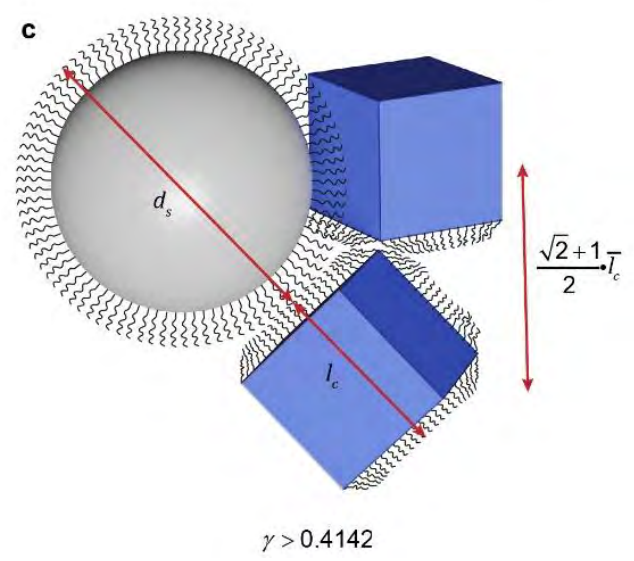

Supplementary Fig. 2 | Representation of the ligand shell around $\mathrm{NCs}$ in binary $\mathrm{ABO}_{3}$ lattice according to hard sphere/cube (a, $\left.\gamma<\gamma_{c, 1}\right)$ and OTM (b and c, $\gamma>\gamma_{c, 1}$ ) models.

The fundamental equation is then obtained by imposing that all $3 c$ cubes are in contact with the spherical NCs in the 1a positions. Note that because these contacts are determined by $l_{c}$ (as opposed to $\bar{l}_{c}$ ), since they cannot be deformed. Thus we obtain for A-B distance (A and B in contact, corresponding to the distance between Wyckoff positions $1 \mathrm{a}$ and $3 \mathrm{c}$ ):

$$
\frac{1+\gamma}{2} d_{s}=\frac{\bar{\gamma}}{\sqrt{2}-1} \frac{\sqrt{2}}{2} d_{s}
$$

leading to OTM formula for the lattice constant:

$$
a_{L, \text { OTM }}(\gamma)=\frac{1+\gamma}{\sqrt{2}} d_{s} \quad \gamma_{c, 1} \leq \gamma<\bar{\gamma}_{c, 2} .
$$

This formula is valid provided that the two conditions

$$
\bar{\gamma} \geq \frac{l_{\text {core }}}{d_{s}}=\bar{\gamma}_{\text {min }}
$$

( $\bar{\gamma}$ must be higher than minimum value $\bar{\gamma}_{\min }$ of $3 c$ cube cores touching at vertices)

$$
\frac{\bar{\gamma}}{2(\sqrt{2}-1)} \geq \gamma
$$

(distance between $1 \mathrm{~b}$ and $3 \mathrm{c} \geq l_{c}$, minimum distance between two cubes) 
Since $\bar{\gamma}_{\text {min }}$ is a function of both the $l_{\text {core }}$ and the NC hard-sphere diameter, it may be expressed as

$$
\bar{\gamma}_{\min }=\frac{l_{\text {core }}}{l_{c}} \gamma \equiv r \gamma
$$

where $r$ is a constant for a cube of fixed core and ligands.

The limit $\bar{\gamma}_{c, 2}$ where Eq. S22 applies is obtained from Eq. S23 combined with Eq. S20:

$$
\bar{\gamma}_{c, 2}=\frac{1}{\frac{\sqrt{2}}{\sqrt{2}-1} r-1}^{r=0.8848} \approx 0.4948
$$

where $r=\frac{8.6}{8.6+1.12}=0.8848$ corresponds to the cubes in the $\mathrm{ABO}_{3}$ experiment. Alternatively, from Eq. S24 combined with Eq. S20 one receives

$$
\bar{\gamma}_{c, 2}=\frac{1}{2 \sqrt{2}-1} \approx 0.5469
$$

Therefore, $\bar{\gamma}_{c, 2}$ is given by Eq. S26 provided that

$$
r \geq r_{\text {crit }} \equiv 2(\sqrt{2}-1) \approx 0.8284
$$

and by Eq. S27 if $r<r_{\text {crit }}$.

The lattice constant for $\gamma \geq \bar{\gamma}_{c, 2}$ is given as

$$
a_{L, O T M}(\gamma)=\left\{\begin{array}{ll}
(\sqrt{2}+1) r \gamma d_{s} & r \geq r_{\text {crit }} \\
2 \gamma d_{s} & r<r_{\text {crit }}
\end{array} .\right.
$$

The packing fraction is given by

$$
\eta_{O T M}^{s c c}(\gamma)= \begin{cases}\frac{\pi}{6}\left(1+\frac{24}{\pi} \gamma^{3}\right) & \gamma<\gamma_{c, 1} \\ \frac{\sqrt{2} \pi}{3(1+\gamma)^{3}}\left(1+\frac{24}{\pi} \gamma^{3}\right) & \gamma_{c, 1} \leq \gamma<\bar{\gamma}_{c, 2} . \\ \frac{\pi}{6(\sqrt{2}+1)^{3} \gamma^{3} r^{3}}\left(1+\frac{24}{\pi} \gamma^{3}\right) & \gamma \geq \bar{\gamma}_{c, 2}\end{cases}
$$

Note that if $r \leq r_{\text {crit }}$, then one must take $r=r_{\text {crit }}$ in Eq. S30. Results are shown in Fig. 1k of the Main Text. Clearly, $\gamma>\gamma_{c, 1}(0.4142)$ packing density in OTM model greatly exceeds a purely hard-sphere/hard-cube approximation for $\mathrm{ABO}_{3}$ structure. The OTM-corrected values for lattice constants now show much better agreement with the experimental values (Supplementary Table 2, last column). 
Supplementary Table 1. List of NCs and their properties. The parameter $\lambda$ is defined in Eq. S17. $d_{s}$ is the hard-sphere diameter, $\boldsymbol{I}_{\boldsymbol{c}}$ the hard-cube edge length.

\begin{tabular}{|c|c|c|c|}
\hline Core & $d_{\text {core }}(\mathbf{n m})$ & $\lambda$ & $\boldsymbol{d}_{\boldsymbol{s}}$ or $\boldsymbol{l}_{\boldsymbol{c}}(\mathbf{n m})$ \\
\hline \multirow{3}{*}{ CsPbBr $_{3}$} & $5.3(4)$ & 0.626 & 6.42 \\
\cline { 2 - 4 } & $8.6(5)$ & 0.386 & 9.72 \\
\hline \multirow{4}{*}{$\mathbf{F e}_{3} \mathbf{O}_{4}$} & $10.2(6)$ & 0.449 & 13.39 \\
\cline { 2 - 4 } & $12.5(7)$ & 0.366 & 15.87 \\
\cline { 2 - 4 } & $14.5(6)$ & 0.316 & 17.97 \\
\cline { 2 - 4 } & $14.7(5)$ & 0.312 & 18.18 \\
\cline { 2 - 4 } & $15.6(7)$ & 0.294 & 19.11 \\
\cline { 2 - 4 } & $16.8(9)$ & 0.273 & 20.36 \\
\cline { 2 - 4 } & $19.5(9)$ & 0.235 & 23.14 \\
\cline { 2 - 4 } & $19.8(10)$ & 0.231 & 23.45 \\
\cline { 2 - 4 } & $20.7(1.1)$ & 0.221 & 24.37 \\
\cline { 2 - 4 } & $21.5(1.4)$ & 0.213 & 25.14 \\
\hline NaGdF $_{4}$ & $25.1(1.4)$ & 0.182 & 28.81 \\
\hline
\end{tabular}


Supplementary Table 2. Summary of the structural parameters for the obtained SLs. The last three columns represent calculated lattice parameters $\left(\boldsymbol{a}_{L}\right)$ based on hard-sphere and hard cube model, their experimental values $\left(\boldsymbol{a}_{\text {exp }}\right)$ obtained from TEM images and also GISAXS $\left({ }^{*}\right)$ and the lattice parameters calculated with OTM approximation $\left(\boldsymbol{a}_{L, \text { oTM }}\right)$.

\begin{tabular}{|c|c|c|c|c|c|c|c|c|}
\hline Structure & $\begin{array}{l}\text { CsPbBr }_{3} \\
l_{\text {core }}(\mathrm{nm})\end{array}$ & $\begin{array}{c}\mathrm{CsPbBr}_{3} \\
I_{c}(\mathrm{~nm})\end{array}$ & $\begin{array}{c}\mathrm{Fe}_{3} \mathrm{O}_{4} \\
d_{\text {core }}(\mathrm{nm})\end{array}$ & $\begin{array}{c}\mathrm{Fe}_{3} \mathrm{O}_{4} \\
d_{H S}(\mathrm{~nm})\end{array}$ & $\gamma$ & $\begin{array}{c}a_{L} \\
(\mathrm{~nm})\end{array}$ & $\begin{array}{l}a_{\text {exp }} \\
(\mathrm{nm})\end{array}$ & $\begin{array}{r}a_{L, O T M} \\
(\mathrm{~nm})\end{array}$ \\
\hline \multirow{6}{*}{$\begin{array}{l}\text { Binary } \\
\mathrm{ABO}_{3}\end{array}$} & $8.6(5)$ & 9.72 & $14.5(6)$ & 17.97 & 0.541 & 23.47 & $20.5^{*}$ & 20.77 \\
\hline & $8.6(5)$ & 9.72 & $15.6(7)$ & 19.11 & 0.509 & 23.48 & 20.7 & 20.78 \\
\hline & $8.6(5)$ & 9.72 & $\begin{array}{c}16.5(9) \\
\mathrm{NaGdF}_{4}\end{array}$ & 20.00 & 0.486 & 23.47 & 21.6 & 21.02 \\
\hline & $8.6(5)$ & 9.72 & $16.8(9)$ & 20.36 & 0.477 & 23.45 & 21.8 & 21.26 \\
\hline & $8.6(5)$ & 9.72 & 19.5(9) & 23.14 & 0.420 & 23.46 & $23.5^{*}$ & 23.23 \\
\hline & $8.6(5)$ & 9.72 & $20.7(1.1)$ & 24.37 & 0.399 & 24.37 & 24.4 & 24.37 \\
\hline \multirow{6}{*}{$\mathrm{NaCl}$} & $8.6(5)$ & 9.72 & $15.6(7)$ & 19.11 & 0.507 & 28.83 & 28.5 & 28.83 \\
\hline & $8.6(5)$ & 9.72 & $19.8(1.0)$ & 23.45 & 0.414 & 33.16 & 33.6 & 33.16 \\
\hline & $8.6(5)$ & 9.72 & $25.1(1.4)$ & 28.81 & 0.337 & 40.74 & 40.9 & 40.74 \\
\hline & $5.3(4)$ & 6.42 & $14.7(5)$ & 18.18 & 0.353 & 25.7 & 25.3 & 25.7 \\
\hline & $5.3(4)$ & 6.42 & $15.6(7)$ & 19.11 & 0.336 & 27.0 & 27.0 & 27.0 \\
\hline & $5.3(4)$ & 6.42 & $19.8(1)$ & 23.45 & 0.274 & 33.2 & 32.9 & 33.2 \\
\hline
\end{tabular}


Supplementary Note 2. Relationship between crystallographic lattice planes and facets in $\mathrm{Cs}_{\mathbf{P b B r}}$ nanocubes

$\mathrm{CsPbBr}_{3} \mathrm{NCs}$ crystallize in perovskite orthorhombic Pnma structure, which can be derived from the ideal perovskite cubic $P m \overline{3} m$ structure (which consists of $3 \mathrm{D}$ network of corner-sharing $\mathrm{PbBr}_{6}$ octahedra and Cs occupying cuboctahedral voids) by a small octahedral tilting. The relationship between cubic and orthorhombic unit cells is shown in the figure below: the orthorhombic cell axis $\boldsymbol{b}_{\text {orth }}$ is parallel to cubic cell axis $\mathbf{b}_{\mathrm{c}}, \mathbf{a}_{\text {orth }}$ aligns to $\left(\mathbf{a}_{\mathbf{c}}+\mathbf{c}_{\mathrm{c}}\right)$ and $\mathbf{c}_{\text {orth }}$ aligns to $\left(\mathbf{c}_{\mathrm{c}}-\mathbf{a}_{\mathbf{c}}\right)$. Due to these relationships the orthorhombic lattice parameters $a_{\text {orth }}$ and $c_{\text {orth }}$ are almost identical: $a_{\text {orth }}=8.2502 \AA \approx \sqrt{2} a_{c}, \quad c_{\text {orth }}=8.2035 \AA \approx \sqrt{2} a_{c}$ and $b_{\text {orth }}=11.7532 \AA \approx 2 a_{c}{ }^{69}$.

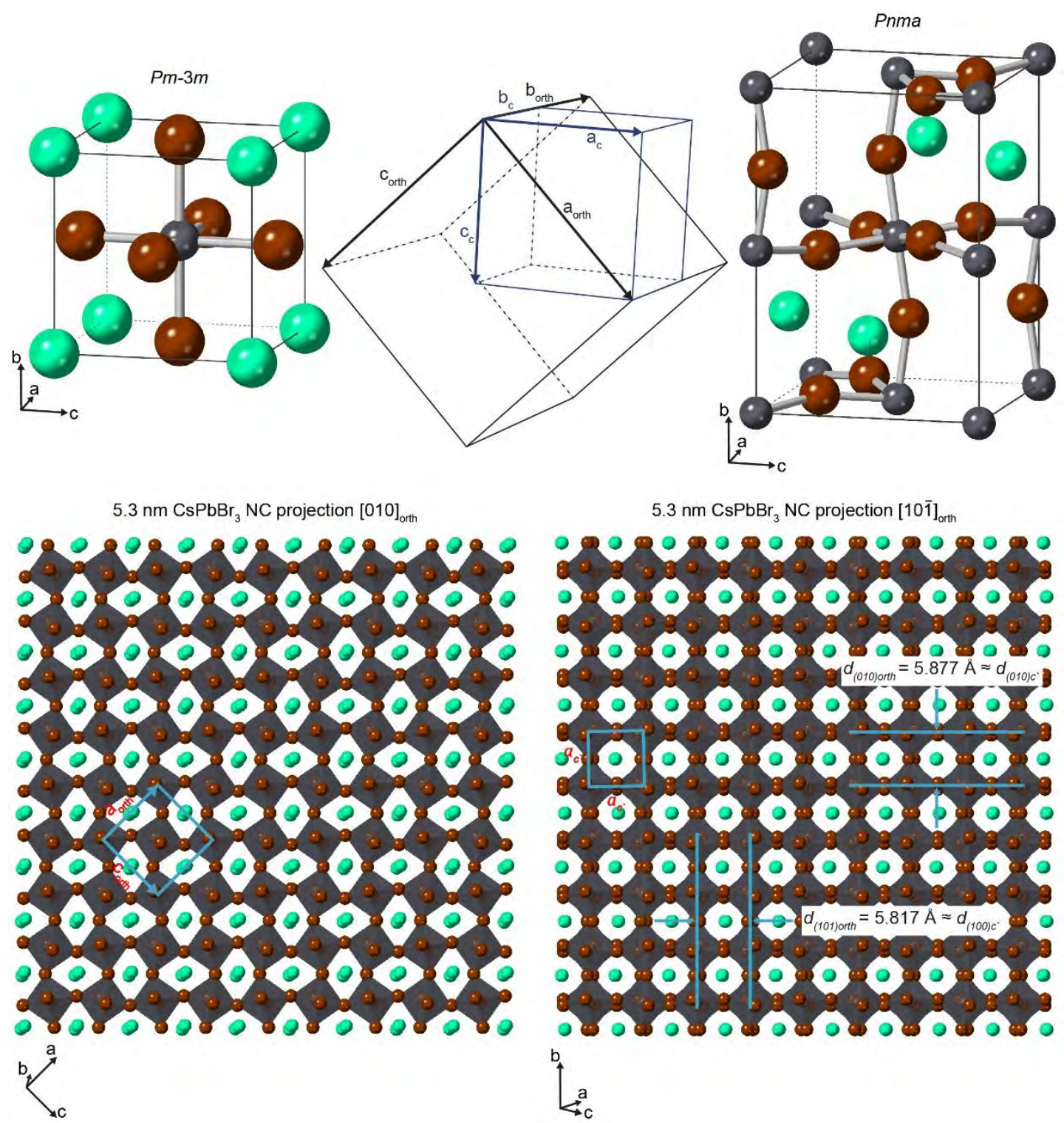

Supplementary Fig. 3 | Relationship between crystallographic lattice planes and facets in $\mathrm{CsPbBr}_{3}$ nanocubes.

$\mathrm{CsPbBr}_{3} \mathrm{NCs}$ are terminated by two $\{010\}$ and four $\{101\}$ lattice planes. Because the difference between $\{010\}$ and $\{101\}$ d-spacings is only $1.0 \%$ and not readily detectable by conventional electron diffraction (ED) we, for the sake of simplicity, assign ED reflections of $\mathrm{CsPbBr}_{3}$ as originated from pseudocubic structure 
with lattice constant $a_{c^{\prime}} \approx 5.85 \AA$ and adopt the notation [100], [010] and [001] referring to $\mathbf{a}_{c^{\prime}}, \mathbf{b}_{\mathrm{c}^{\prime}}$ and $\mathbf{c}_{\mathrm{c}^{\prime}}$ cubic crystallographic axes ${ }^{70,71}$.

Given the small structural difference between all $\{100\}$ lattice planes and natural propensity of lead cation to complete octahedral coordination, all six facets of $\mathrm{CsPbBr}_{3} \mathrm{NC}$ s tend to be AX'-terminated (where $\mathrm{A}-\mathrm{Cs}^{+}$, oleylamonium, didodecyldimethylammonium, $\mathrm{X}^{\prime}-\mathrm{Br}$, oleate). Consequently, the growth of all $\{100\}$ lattice planes in six directions is similar and exceeds the growth in other directions, which makes the nontruncated cubic shape the most stable for lead halide perovskites ${ }^{72}$, contrary to other cubic NCs, for instance, cubic lead sulfide, which vertices and edges are bevelled by (111) and (110) lattice planes, respectively ${ }^{73,74}$. 


\section{Supplementary Note 3. GISAXS characterization of SLS}

Indexing of the binary SL peaks was done manually for the case of a grazing incidence geometry (noted by white markers in Supplementary Fig. 4 and 7f). Here, X-rays are scattered by the binary SLs either prior to or subsequent to refraction with the sample substrate. As scattering images were taken at low incidence angles of $0.04^{\circ}$, we also have to account for a partial scattering contribution stemming from (Lauetype) transmission-diffraction (noted by red markers in Supplementary Fig. 4 and 7f). Here, Laue-type diffraction occurs if X-rays are scattered directly by the binary SLs and are not refracted by the sample substrate.

Scattering images of the $\mathrm{ABO}_{3}$-type binary SL comprising $8.6 \mathrm{~nm} \mathrm{CsPbBr} 3$ and $19.5 \mathrm{~nm} \mathrm{Fe} \mathrm{F}_{4} \mathrm{NCs}$ $(\gamma=0.420)$ on silicon nitride membrane show a regular and highly symmetric scattering pattern (see Fig. 1c from the Main Text). All reflections can be indexed using a simple-cubic $(P m \overline{3} m)$ symmetry with $24.2 \mathrm{~nm}$ unit cell dimension (see Supplementary Fig. 4a). Here, we do not observe the absence of any distinct peak families, which would be a characteristic sign of a higher symmetry packing. Note that $\mathrm{CsPbBr}_{3}$ and $\mathrm{Fe}_{3} \mathrm{O}_{4}$ have different mean electron density and hence different scattering length contrast in the X-ray scattering measurement. An e.g. body-centered cubic (bcc) structural motif, where $\mathrm{Fe}_{3} \mathrm{O}_{4} \mathrm{NCs}$ occupy corner points and $\mathrm{CsPbBr}_{3} \mathrm{NCs}$ reside at the body-centered position, would hence still give the scattering pattern of a simple cubic motif where only the (phase-terms determining) relative peak intensities are affected. Furthermore, assuming a bcc arrangement of $\mathrm{Fe}_{3} \mathrm{O}_{4} \mathrm{NCs}$, intercalation of $\mathrm{CsPbBr}_{3} \mathrm{NCs}$ with site-dependent orientation would break the bcc symmetry such that only the simple-cubic motif remains.
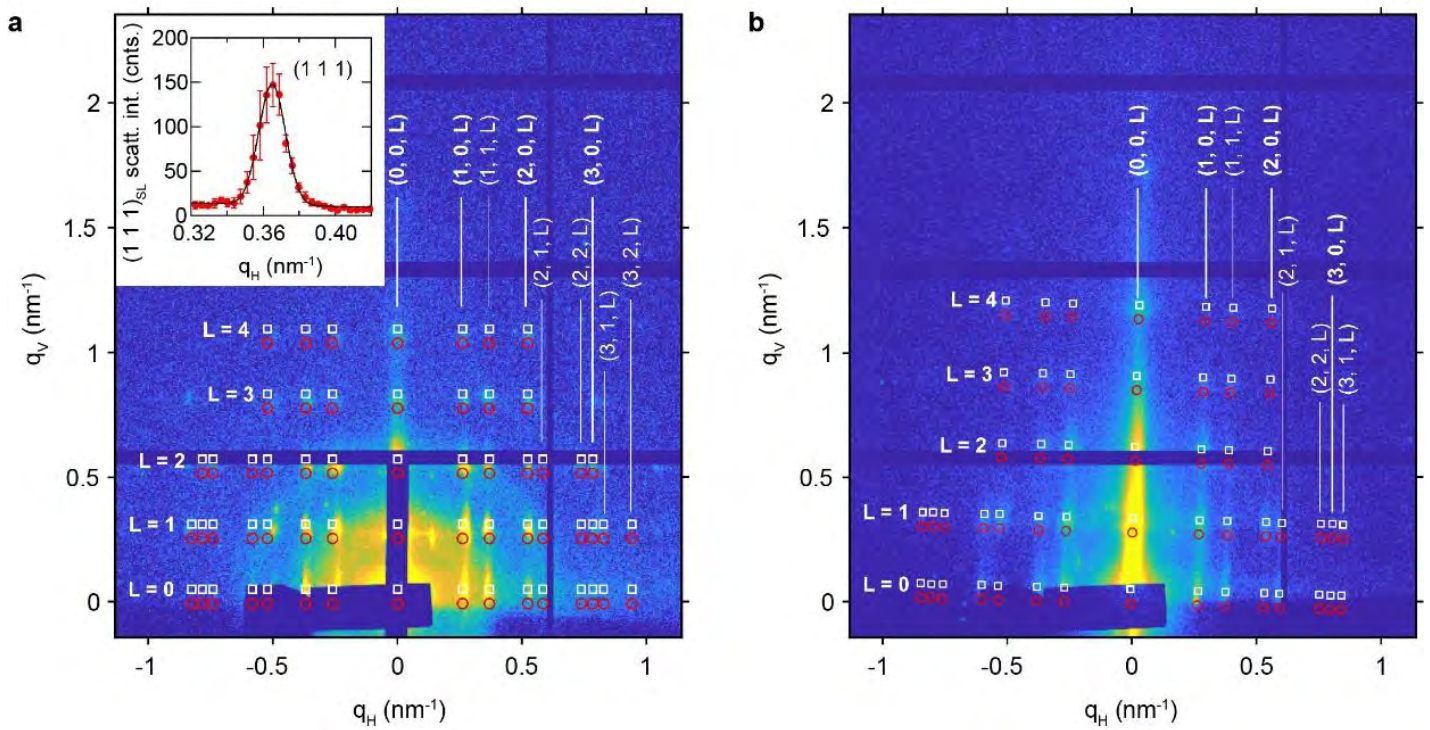

Supplementary Fig. 4 | Indexing of GISAXS scattering images from $\mathrm{ABO}_{3}$-type binary SL comprising $8.6 \mathrm{~nm} \mathrm{CsPbBr}$ and $19.5 \mathrm{~nm} \mathrm{Fe}_{3} \mathrm{O}_{4} \mathrm{NCs}$. a, The binary SL self-assembled on a silicon nitride membrane corresponds to an undistorted cubic lattice with $a=b=c=24.2 \mathrm{~nm}$. The inset shows the Gaussian peak fit $\left(F W H M=0.0174 \mathrm{~nm}^{-1}\right.$ ) of an in-plane cut (average over $n=10$ pixels, corresponding to $\Delta q_{v}=0.02 \mathrm{~nm}^{-1}$ ) through the $(1,1,1)$ reflection to determine the coherent in-plane domain size of the binary SL (error bars denote the standard-deviation of the scattering intensity along $q_{v}$ over $n=10$ pixels within the horizontal cut). $\mathbf{b}$, The scattering image of the same system self-assembled on a TEM grid can only be indexed using an out-of-plane compressed tetragonal unit cell. White markers correspond to the grazing-incidence diffraction-peak positions and red markers show the corresponding Laue-type diffraction pattern. 
To quantify the degree of crystalline order, we determine the size of the coherent scattering volume, which is directly related to the crystalline domain size. Following the Scherrer equation, we determine the peak width (FWHM) of the isolated (1 111 ) sL reflection (which is not distorted by any other diffuse scattering contribution) with $\Delta q=0.0174 \mathrm{~nm}^{-1}$, which corresponds to a mean in-plane domain size of $2 \pi / \Delta q \approx 361 \mathrm{~nm}$.

The same $\mathrm{ABO}_{3}$-type binary SL comprising $8.6 \mathrm{~nm} \mathrm{CsPbBr} 3$ and $19.5 \mathrm{~nm} \mathrm{Fe} 3 \mathrm{O}_{4} \mathrm{NCs}(\gamma=0.420)$ was also self-assembled on a TEM grid. Here, the GISAXS scattering pattern can only be indexed using a tetragonal geometry $(P 4 / \mathrm{mmm})$, with slight out-of-plane compression $[a=b=23.5 \mathrm{~nm} ; c=22.0 \mathrm{~nm}]-$ see Supplementary Fig. 4b. This out-of-plane lattice compression is likely a side effect of the solvent-evaporation procedure, creating an anisotropic concentration gradient and hence uniaxial thermodynamic pressure. Indeed, the same effect is known to occur in binary SL of classical spherical NCs ${ }^{75}$. 


\section{Supplementary Note 4. Superfluorescence in various binary superlattices}

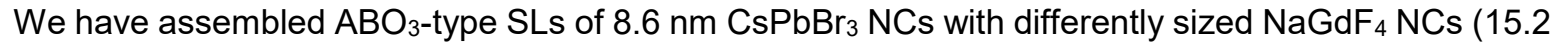
$\mathrm{nm}$ and $19.5 \mathrm{~nm}$ ), and compared their optical properties with a NaCl-type SL formed by the assembly of 8.6 $\mathrm{nm} \mathrm{CsPbBr} 3 \mathrm{NCs}_{3}$ and $18.6 \mathrm{~nm} \mathrm{NaGdF}{ }_{4} \mathrm{NCs}$ by performing ultrafast optical spectroscopy at cryogenic temperatures. These samples were ideally suited for such a comparison as they were phase-pure; that is, where only a specific $\mathrm{SL}\left(\mathrm{ABO}_{3}\right.$ or $\left.\mathrm{NaCl}\right)$ is found macroscopically across the entire sample, without a substantial amount of disordered regions or other phases (both single-component and binary). We have found that $\mathrm{SF}$ is absent in $\mathrm{NaCl}$-type SLs, while two $\mathrm{ABO}_{3}$-type SLs clearly exhibit the typical burst emission of SF of two different kinds, namely blue and red-shifted with respect to excitonic PL, as discussed below and illustrated in Supplementary Fig. 23.

In addition to the different arrangements of the emitting dipoles, the drastic difference between $\mathrm{ABO}_{3}-$ and $\mathrm{NaCl}$-type SLs in achieving SF can be due to the much lower effective NC density in NaCl-type SLs ( $\mathrm{CsPbBr}_{3} \mathrm{NCs}$ concentration of $1.18 \times 10^{5} \mu \mathrm{m}^{-3}$, perovskite $\mathrm{NC}$ volume fraction of 0.0748 ), which are characterized by a $1: 1$ ratio between emitting perovskite NCs and $\mathrm{NaGdF}_{4} \mathrm{NCs}$. In $\mathrm{ABO}_{3}$-type SLs (4:1 ratio), the higher perovskite NC density $\left(4.58 \times 10^{5} \mu^{-3}\right.$, perovskite NC volume fraction of 0.291$)$ and the much reduced NC-to-NC distance can facilitate the occurrence of coherent coupling. In fact, NaCl-type SLs only show the characteristic emission from neutral excitons and trions (Supplementary Fig. 23a), while the radiative rates remain almost unchanged for higher fluences (Supplementary Fig. 23b, c). On the contrary, $\mathrm{ABO}_{3}$-type SLs clearly show a significant shorting of the radiative lifetime down to few picoseconds with the appearance of oscillations in the time domain (Supplementary Fig. 23e, f). Furthermore, by exploring $\mathrm{ABO}_{3}-$ type SLs composed of large, $19.5 \mathrm{~nm} \mathrm{NaGdF}_{4} \mathrm{NCs}\left(3.16 \times 10^{5} \mu \mathrm{m}^{-3}\right.$, perovskite NC volume fraction of 0.201), we found that SF can occur almost resonantly (actually, somewhat blue-shifted) with the exciton transition (Supplementary Fig.23 g-i), unlike to a red-shifted SF in the case of smaller $\mathrm{NaGdF}_{4} \mathrm{NCs}_{\text {. We }}$ have two hypotheses for this different SF behaviour. One possibility is that since almost an order of magnitude higher fluence is required for its occurrence (Supplementary Fig. 23g-i), we believe that this could be due to SF from biexcitons, as reported for $\mathrm{CuCl}$ QDs embedded in a $\mathrm{NaCl}$ matrix ${ }^{76}$. Having increased the perovskite NC-to-NC distance by employing larger $\mathrm{NaGdF}_{4} \mathrm{NCs}$, the coherent coupling among several NCs could be reduced in the single exciton regime but still occurs through the interaction of biexcitons, given their higher oscillator strength. Recent experiments which combine fluence-dependent transient absorption spectroscopy with a robust spectral deconvolution method unveiled the repulsive character of excitonexciton interactions in $\mathrm{CsPbBr}_{3} \mathrm{NCs}^{77}$, which would be in line with our observations of a small blue-shift. However, fluence-dependent experiments do not show the typical power-law behavior expected for biexcitons (power-law exponent of 2), suggesting that the Auger process might alter the dynamics. Alternatively, a second hypothesis is that SF still emerges from single excitons where the NCs are heated by the strong excitation pulse to emit at slightly higher energy (as also observed for the non-SF NaCl-type

SLs). The increased fluence onset can then be related to the increased energetic disorder present at higher energy due to quantum confinement. 


\section{Supplementary Figures 5-14}
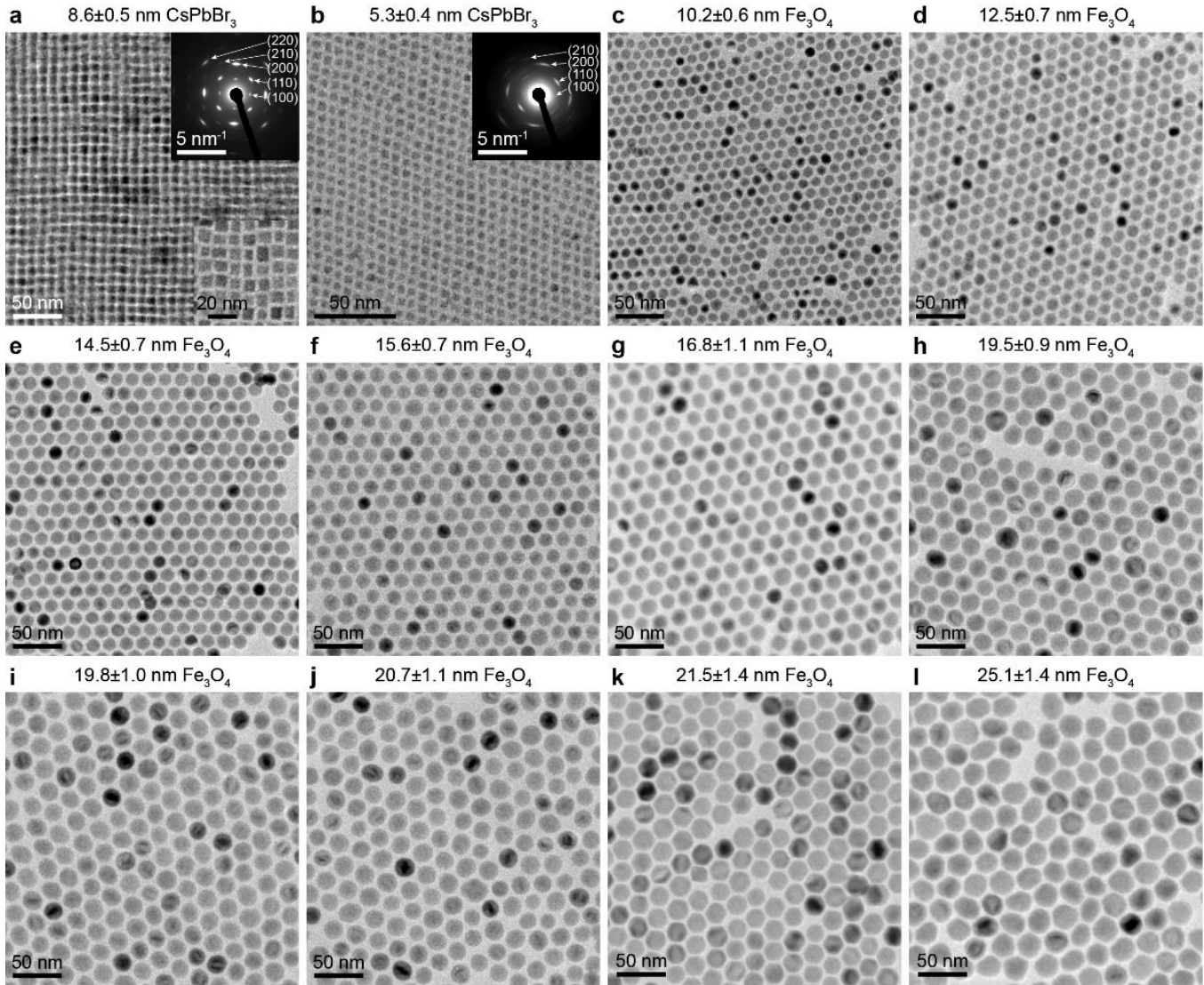

h $\quad 19.5 \pm 0.9 \mathrm{~nm} \mathrm{Fe} \mathrm{O}_{4}$

g $\quad 16.8 \pm 1.1 \mathrm{~nm} \mathrm{Fe} \mathrm{O}_{4}$
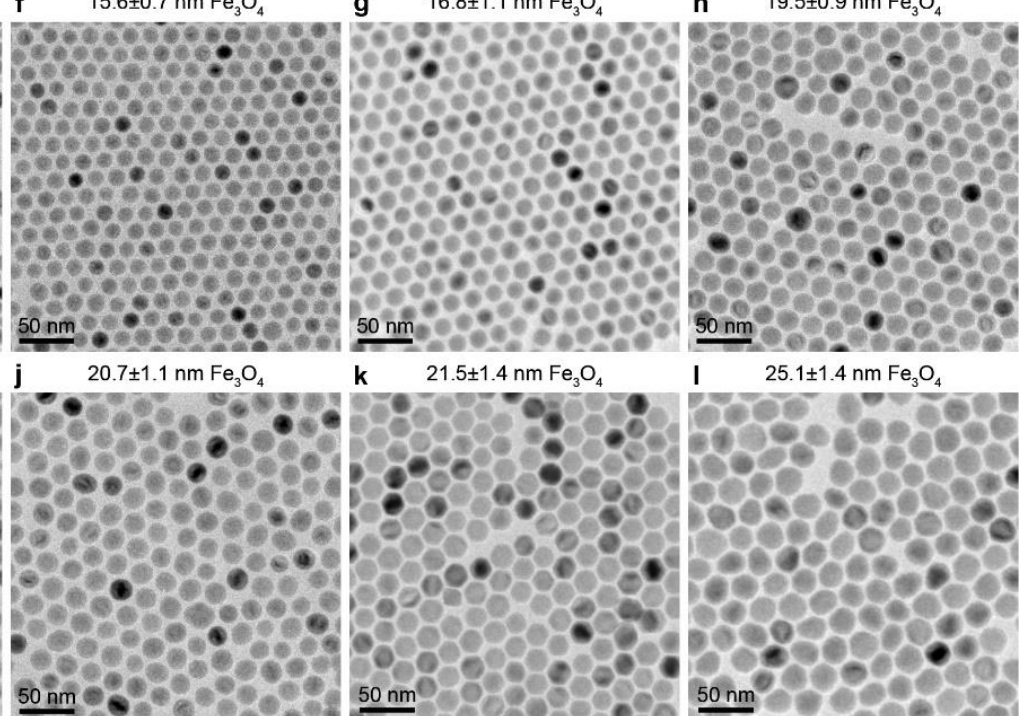

I $\quad 25.1 \pm 1.4 \mathrm{~nm} \mathrm{Fe}_{3} \mathrm{O}_{4}$
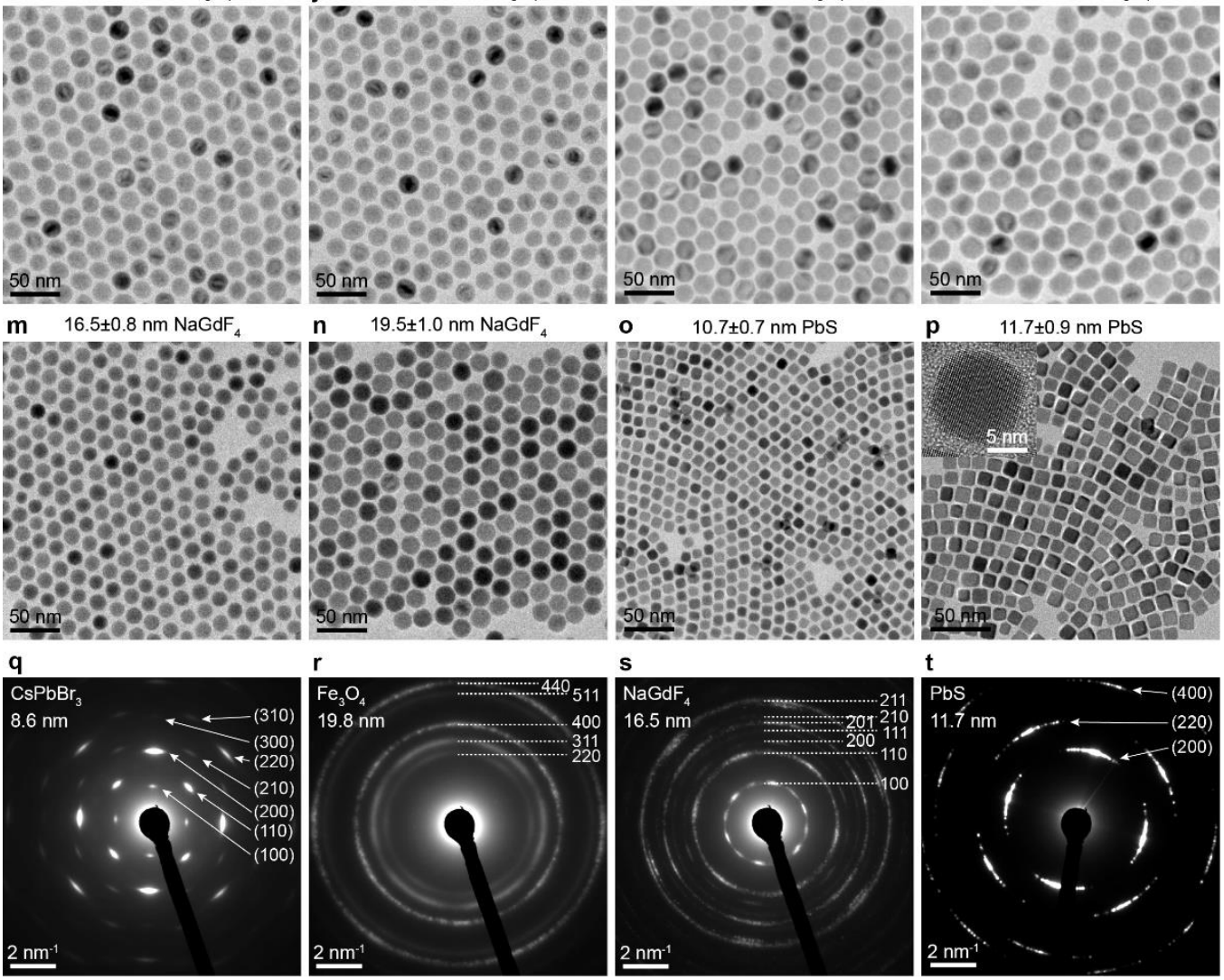

Supplementary Fig. 5 | TEM images and ED patterns of monodisperse NC building blocks used for self-assembly. a, b, DDAB-treated $\mathrm{CsPbBr}_{3} \mathrm{NCs}$. c-I, oleate-capped $\mathrm{Fe}_{3} \mathrm{O}_{4} \mathrm{NCs}$. m, $\mathbf{n}$, oleate-capped $\mathrm{NaGdF}_{4}$ NCs. o, p, oleate-capped PbS NCs. q-t, Corresponding selected area wide-angle electron diffraction (ED) patterns of these NCs. NC size-distribution was in the range from 4.2 to $7.5 \%$ (standard size deviation) and was calculated based on 300 particles. ED patterns of $\mathrm{CsPbBr}_{3} \mathrm{NC}$ s confirm perovskite phase. The presence of diffraction spots, instead of continuous powder rings, indicates the consistency of NCs orientation and the alignment of $\langle 100\rangle$ crystallographic directions of nanocubes with the [001] zone axis parallel to an electron beam. 

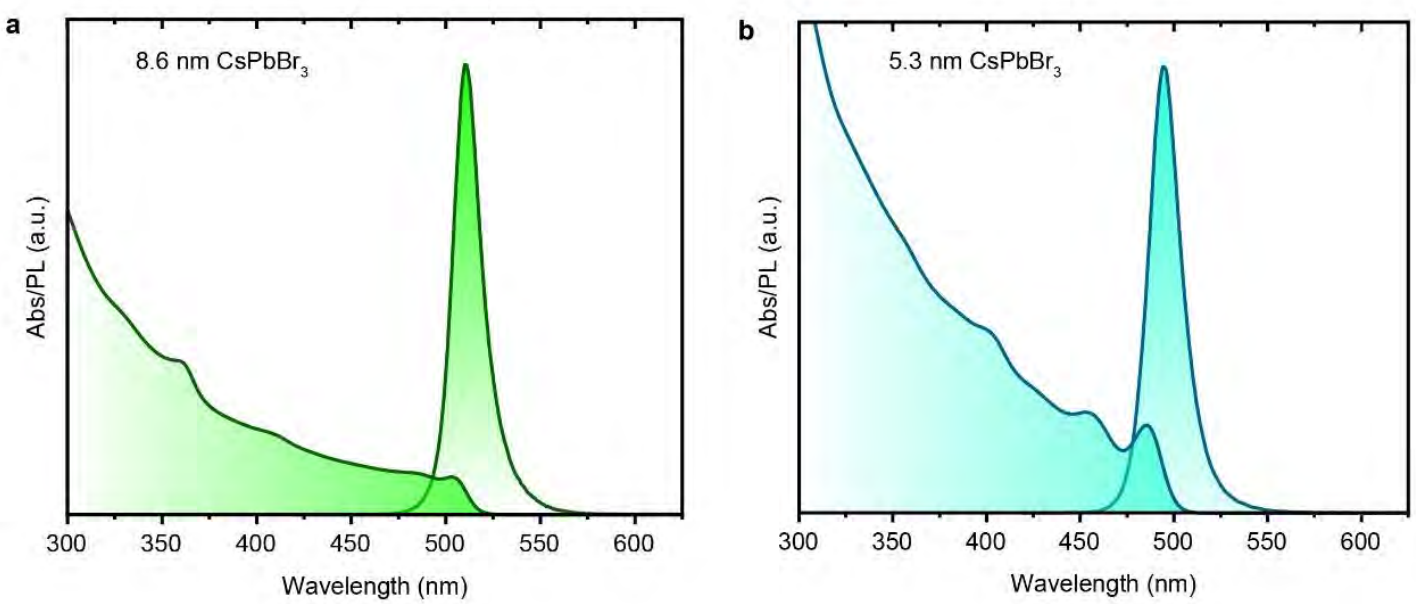

Supplementary Fig. 6 | Optical properties of colloidal $\mathrm{CsPbBr}_{3} \mathrm{NCs}$ dispersed in toluene. a, Absorption and $\mathrm{PL}$ spectra of $8.6 \mathrm{~nm} \mathrm{CsPbBr} 3 \mathrm{NCs}$ (PL quantum yield $>90 \%$ ). b. Absorption and PL spectra of $5.3 \mathrm{~nm} \mathrm{CsPbBr} 3 \mathrm{NCs}$ (PL quantum yield > 55\%). 
Binary $\mathrm{ABO}_{3}$-type $\mathrm{SLs}$

$8.6 \mathrm{~nm} \mathrm{CsPbBr}$ and $14.5 \mathrm{~nm} \mathrm{Fe} \mathrm{O}_{4}$
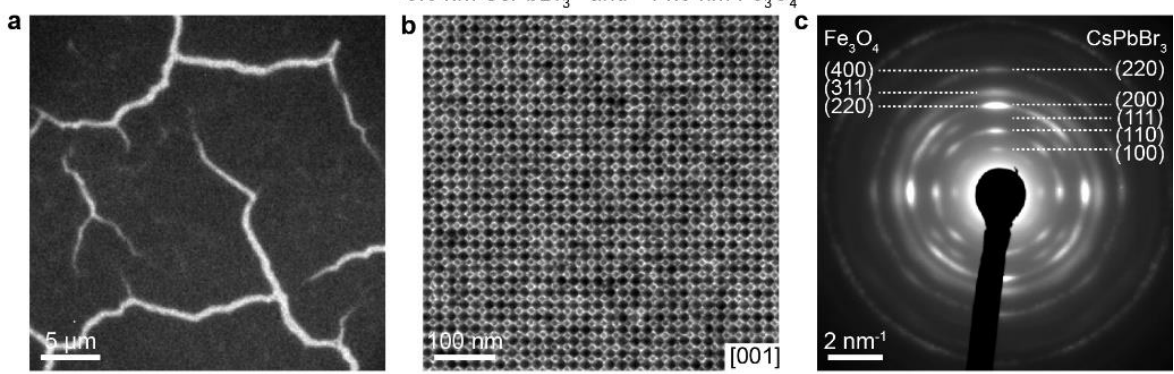

$8.6 \mathrm{~nm} \mathrm{CsPbBr}$ and $15.6 \mathrm{~nm} \mathrm{Fe} \mathrm{O}_{4}$
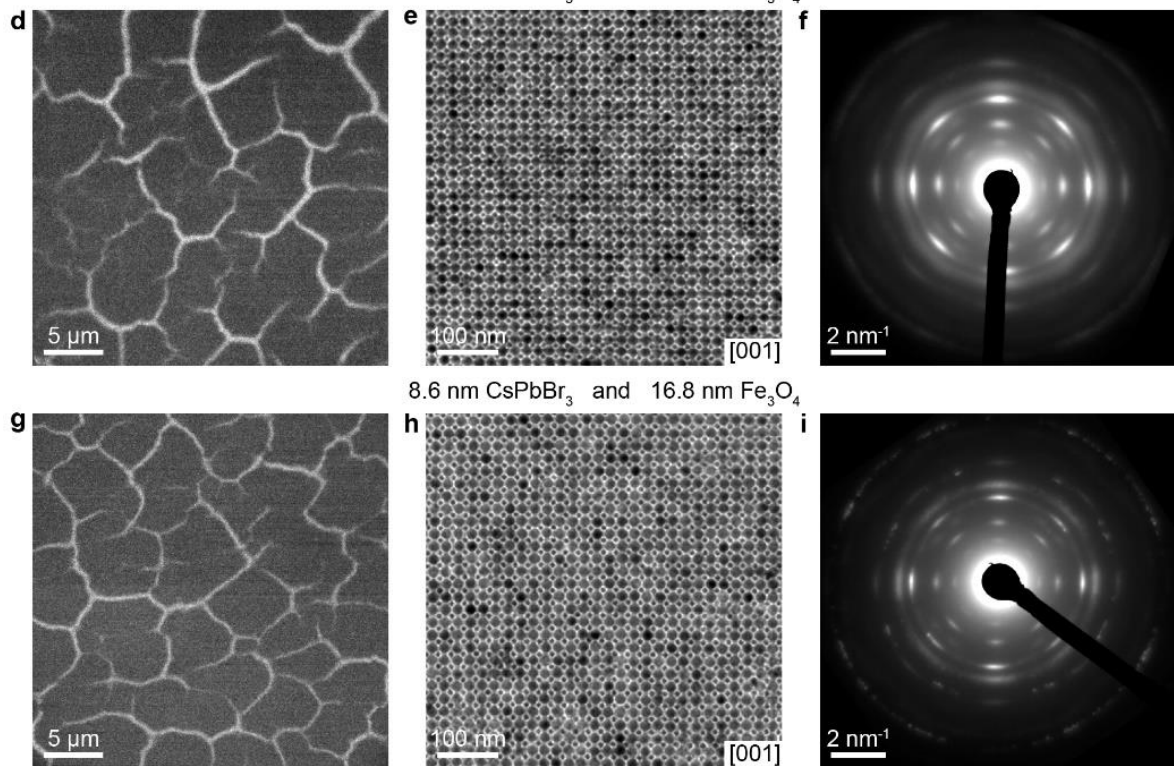

$8.6 \mathrm{~nm} \mathrm{CsPbBr}$ and $16.8 \mathrm{~nm} \mathrm{Fe}_{3} \mathrm{O}_{4}$

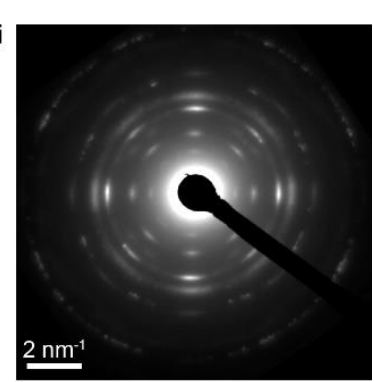

$8.6 \mathrm{~nm} \mathrm{CsPbBr}$ and $19.5 \mathrm{~nm} \mathrm{Fe} \mathrm{O}_{4}$
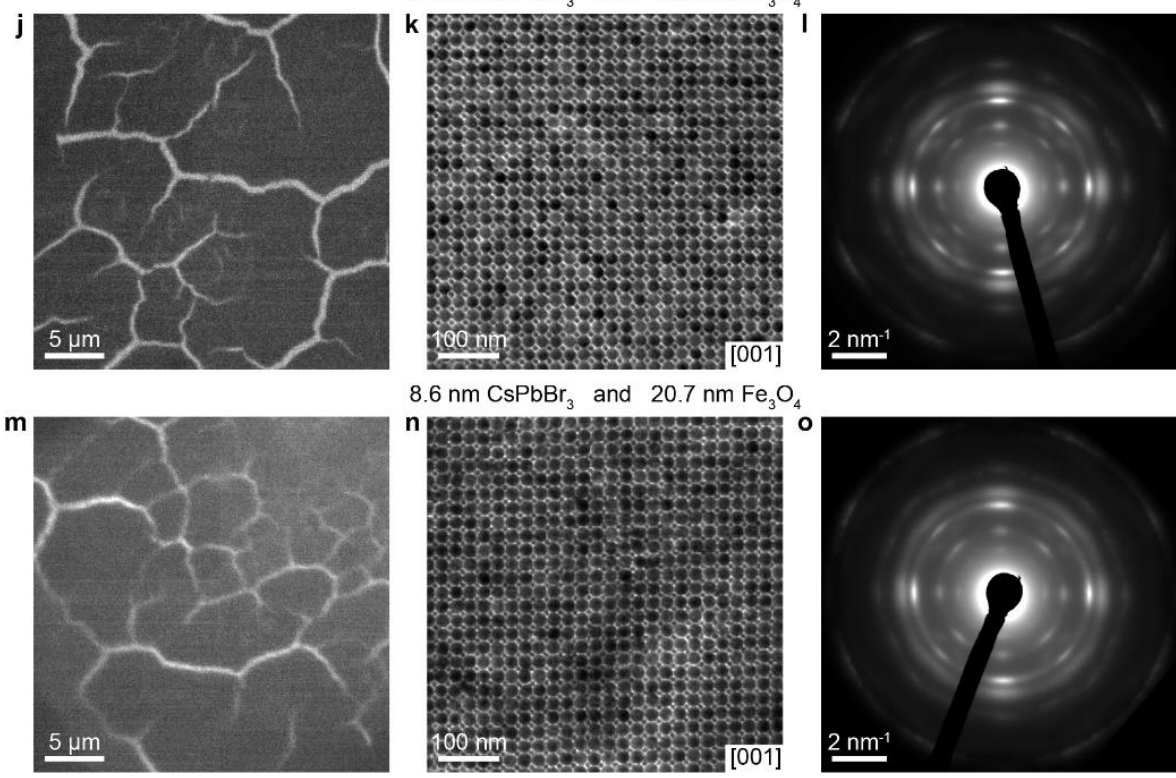

$8.6 \mathrm{~nm} \mathrm{CsPbBr}$ and $20.7 \mathrm{~nm} \mathrm{Fe} \mathrm{O}_{4}$
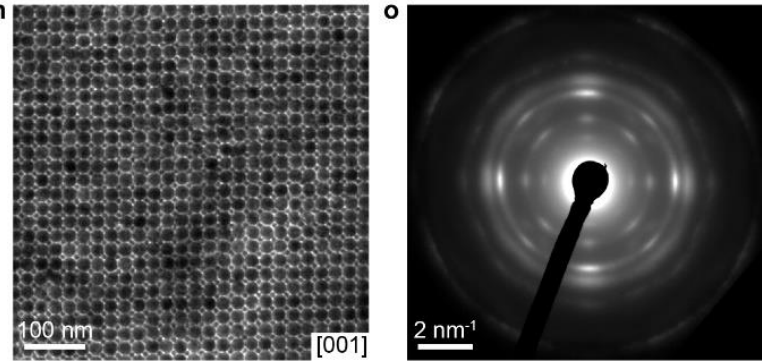

Supplementary Fig. 7 | Binary $\mathrm{ABO}_{3}$-type SLs comprising $8.6 \mathrm{~nm} \mathrm{CsPbBr} 3 \mathrm{NCs}$ and differently sized spherical $\mathrm{Fe}_{3} \mathrm{O}_{4} \mathrm{NCs}(\mathbf{1 4 . 5 - 2 0 . 7} \mathbf{n m})$. a-c, $\gamma=0.541$. d-f, $\gamma=0.509$. $\mathbf{g - i}, \gamma=0.486$. j-I, $\gamma=0.420$. $\mathbf{m}-\mathbf{0}$, $\gamma=0.399$. Distinct ED reflections from B- and O-positioned $\mathrm{CsPbBr}_{3} \mathrm{NCs}$ are maintained across all samples. 

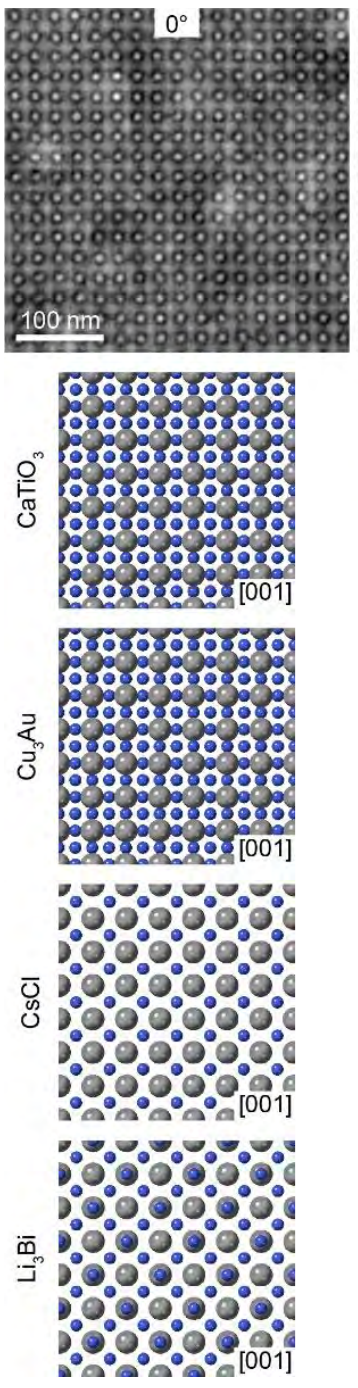
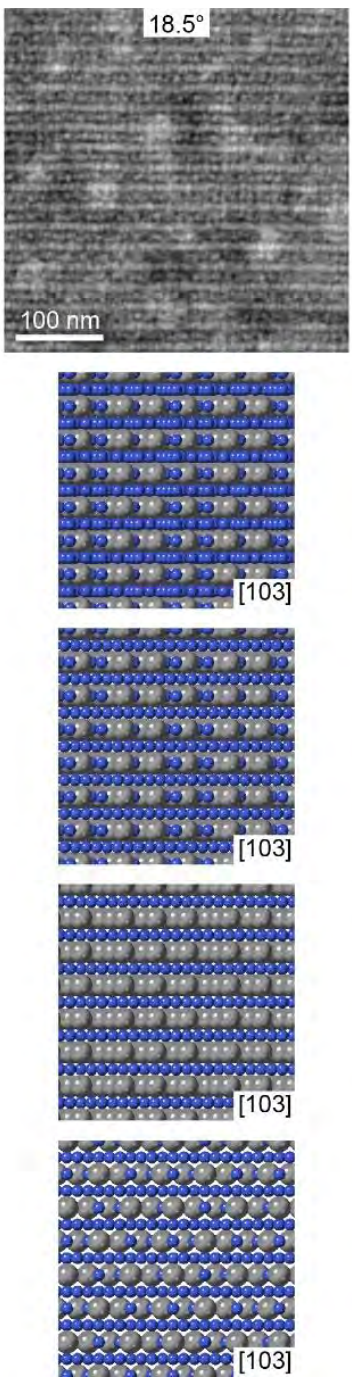
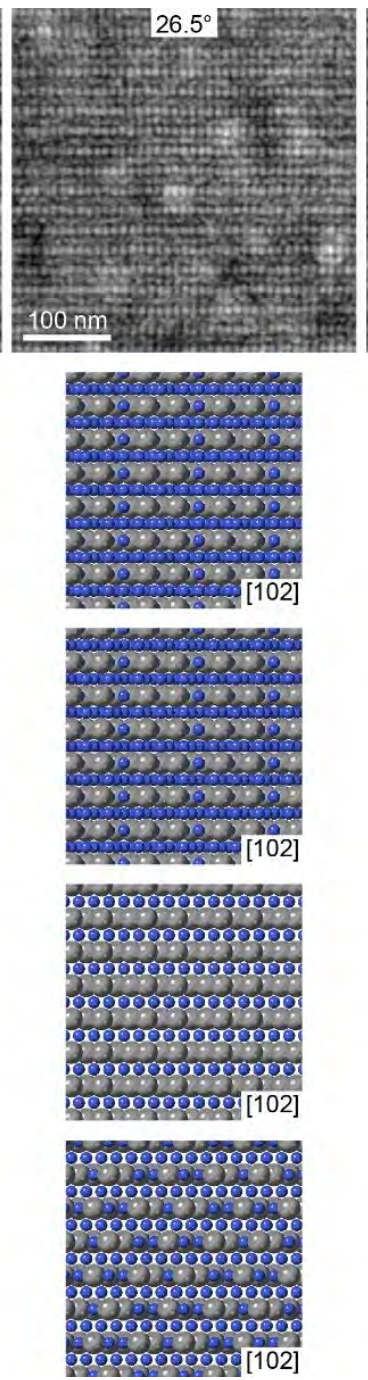
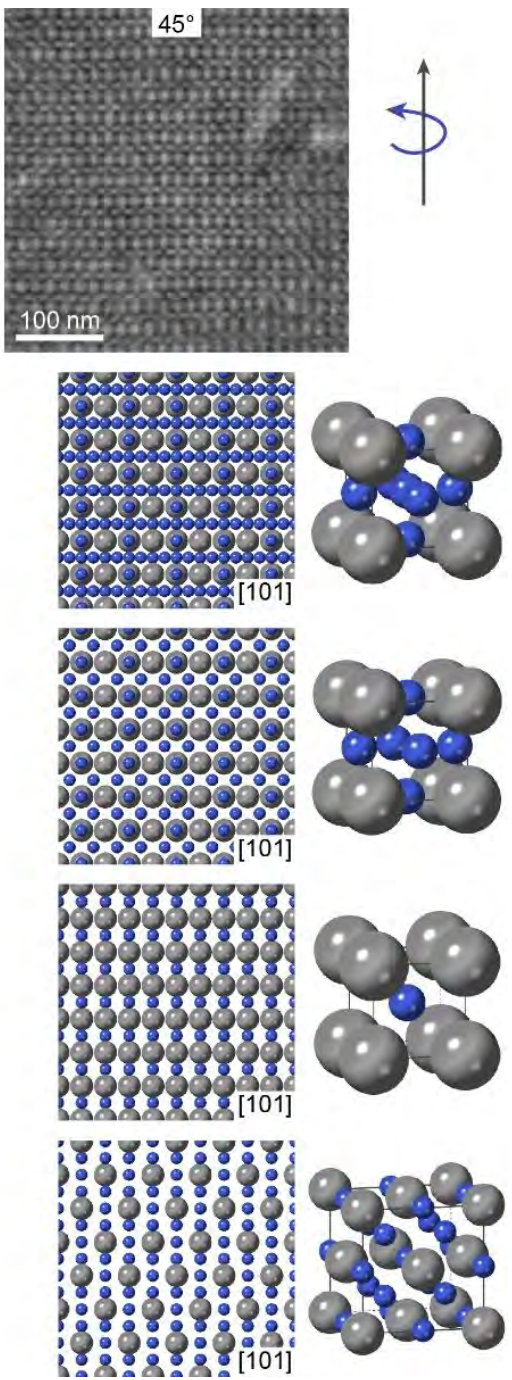

Supplementary Fig. 8 | HAADF-STEM tilting series around the [010] $]_{\mathrm{SL}}$ axis for binary $\mathrm{ABO}_{3}$-type SL comprising $8.6 \mathrm{~nm} \mathrm{CsPbBr}$ and $19.5 \mathrm{~nm} \mathrm{Fe} \mathrm{O}_{4} \mathrm{NCs}$. HAADF-STEM images of a SL domain at different tilting angles are compared with the corresponding projections of $\mathrm{CaTiO}_{3}, \mathrm{Cu}_{3} \mathrm{Au}, \mathrm{CsCl}$ and $\mathrm{Li}_{3} \mathrm{Bi}$ structures. 

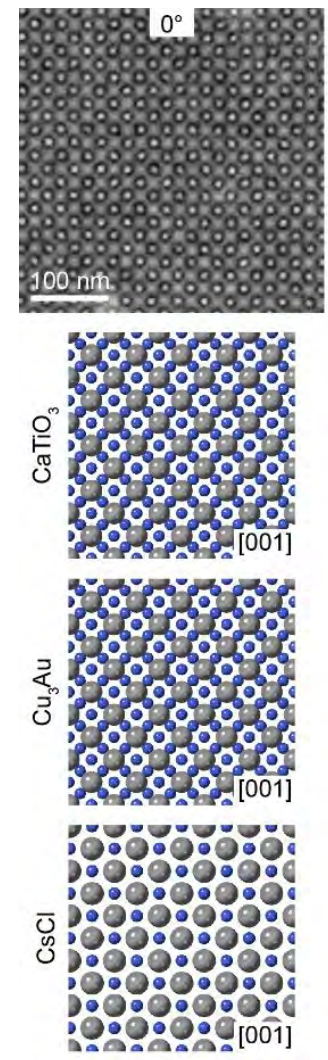

:00 10.9.0.0.0. - 10.9-9.9. $\bar{m}_{m}, 00000000$

I 10000000 :001]
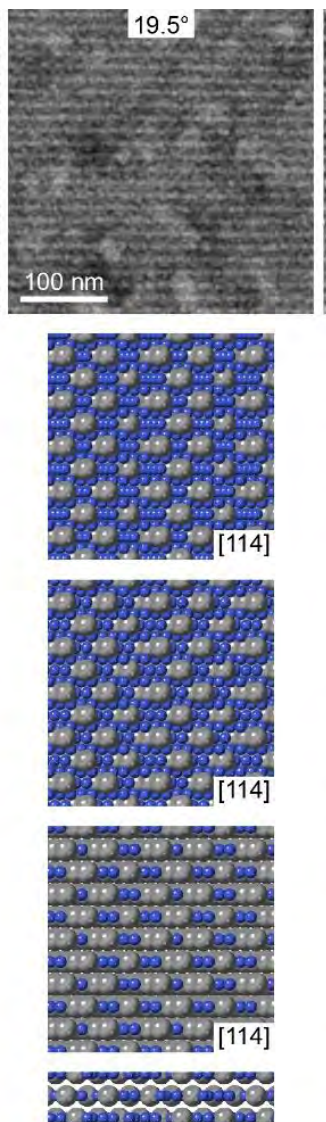

- 0200000 0000000000 rer 200000000 Doosopor 3. 2000000 [114]
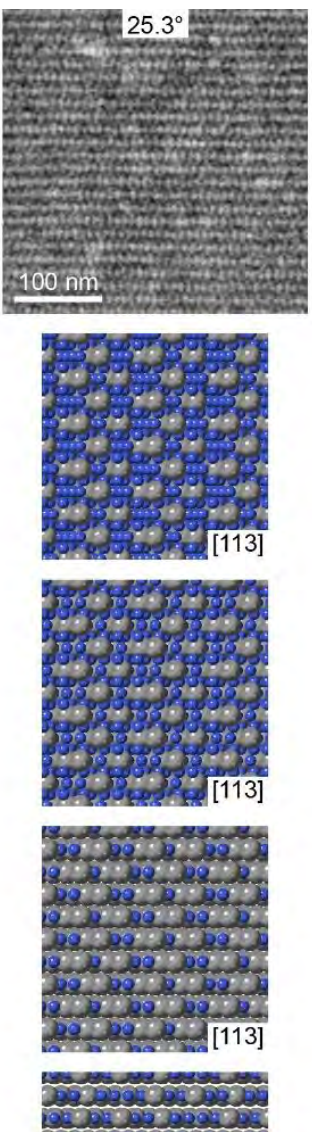

No.

33.

n.

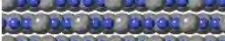

20202950509209

19205039.2395.93

29.3905.090[113]
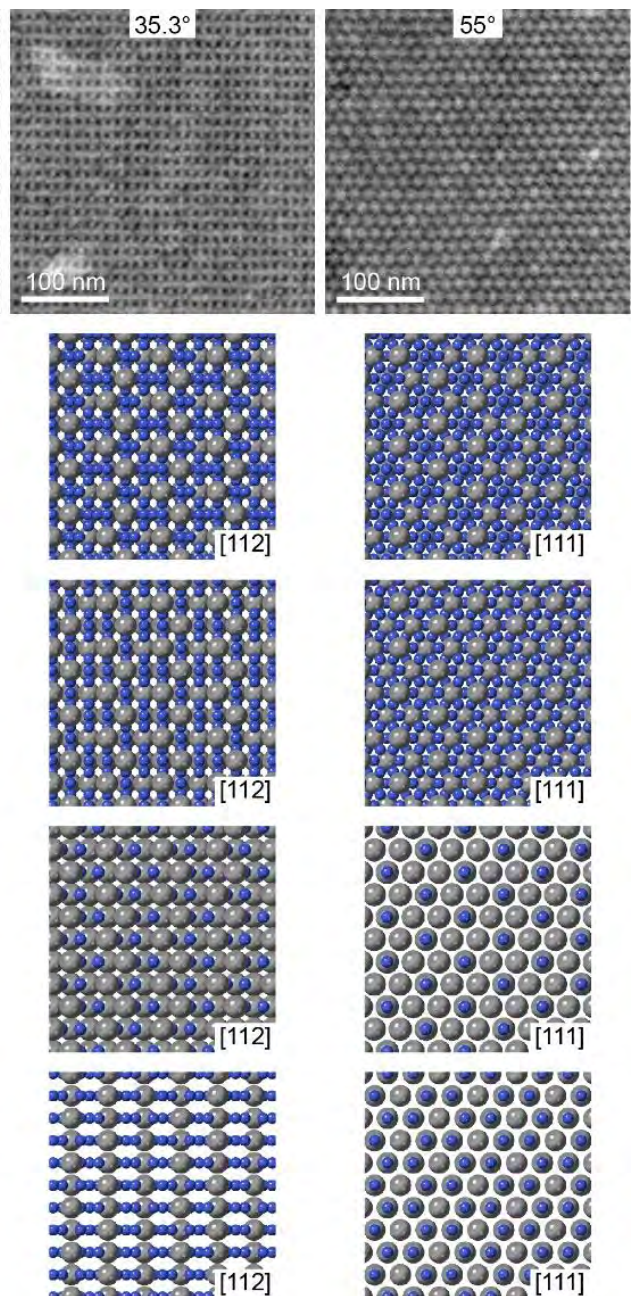

00000000 \% 0009000 00000 10000090 0000000 0000. [111]

100000\% 00000 0000000 -19090090 0000000\% 10000090 1000000 1000000 -1000 [111]

Supplementary Fig. 9 | HAADF-STEM tilting series around the [110] $]_{\mathrm{SL}}$ axis for binary $\mathrm{ABO}_{3}$-type SL comprising $8.6 \mathrm{~nm} \mathrm{CsPbBr}$ and $19.5 \mathrm{~nm} \mathrm{Fe} \mathrm{O}_{4} \mathrm{NCs}$. HAADF-STEM images of a SL domain at different tilting angles are compared with the corresponding projections of $\mathrm{CaTiO}_{3}, \mathrm{Cu}_{3} \mathrm{Au}, \mathrm{CsCl}$ and $\mathrm{Li}_{3} \mathrm{Bi}$ structures. 

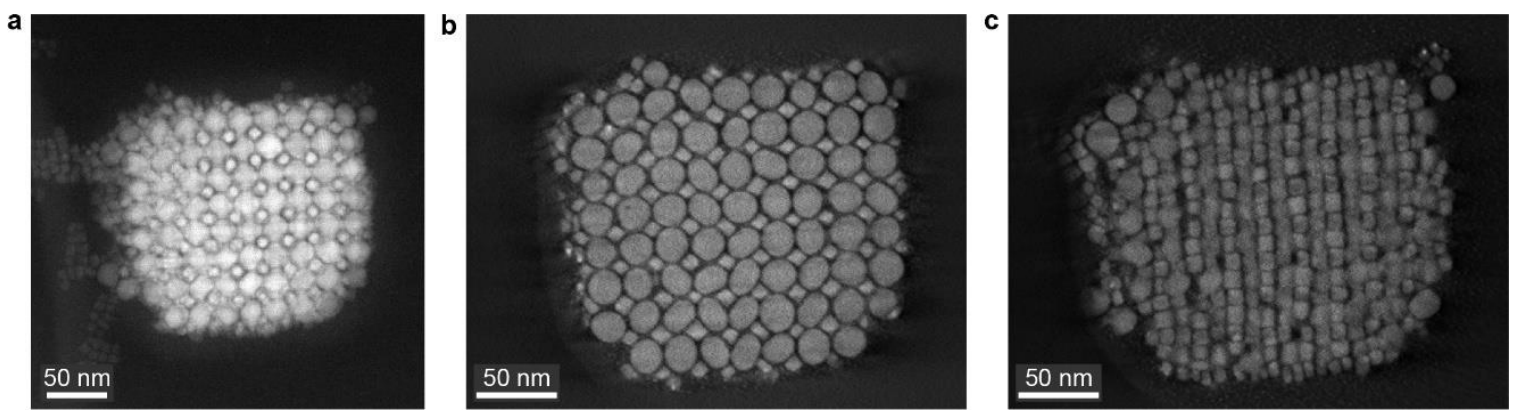

Supplementary Fig. 10 | Tomographic reconstruction of binary $\mathrm{ABO}_{3}$-type $\mathrm{SL}$ comprising $8.6 \mathrm{~nm}$ $\mathrm{CsPbBr}_{3}$ and $19.5 \mathrm{~nm} \mathrm{NaGdF}{ }_{4} \mathrm{NCs}$. a, HAADF-STEM image of SL domain. b, Reconstruction of a volume slice through the (001)sL lattice plane confirms the orientation of O-site cubes that two of the $<110\rangle$ directions of $\mathrm{CsPbBr}_{3}$ are aligned with $\left\langle 100>_{\mathrm{SL}}\right.$. c, Reconstruction of a volume slice through the (002)sL lattice plane visualizes the orientation of $\mathrm{B}$-site cubes $\left(45^{\circ}\right.$ rotated compared to O-site cubes) with the $\langle 100\rangle$ directions of $\mathrm{CsPbBr}_{3}$ aligned with $\langle 100\rangle_{\mathrm{SL}}$. See also Supplementary Video 1. 

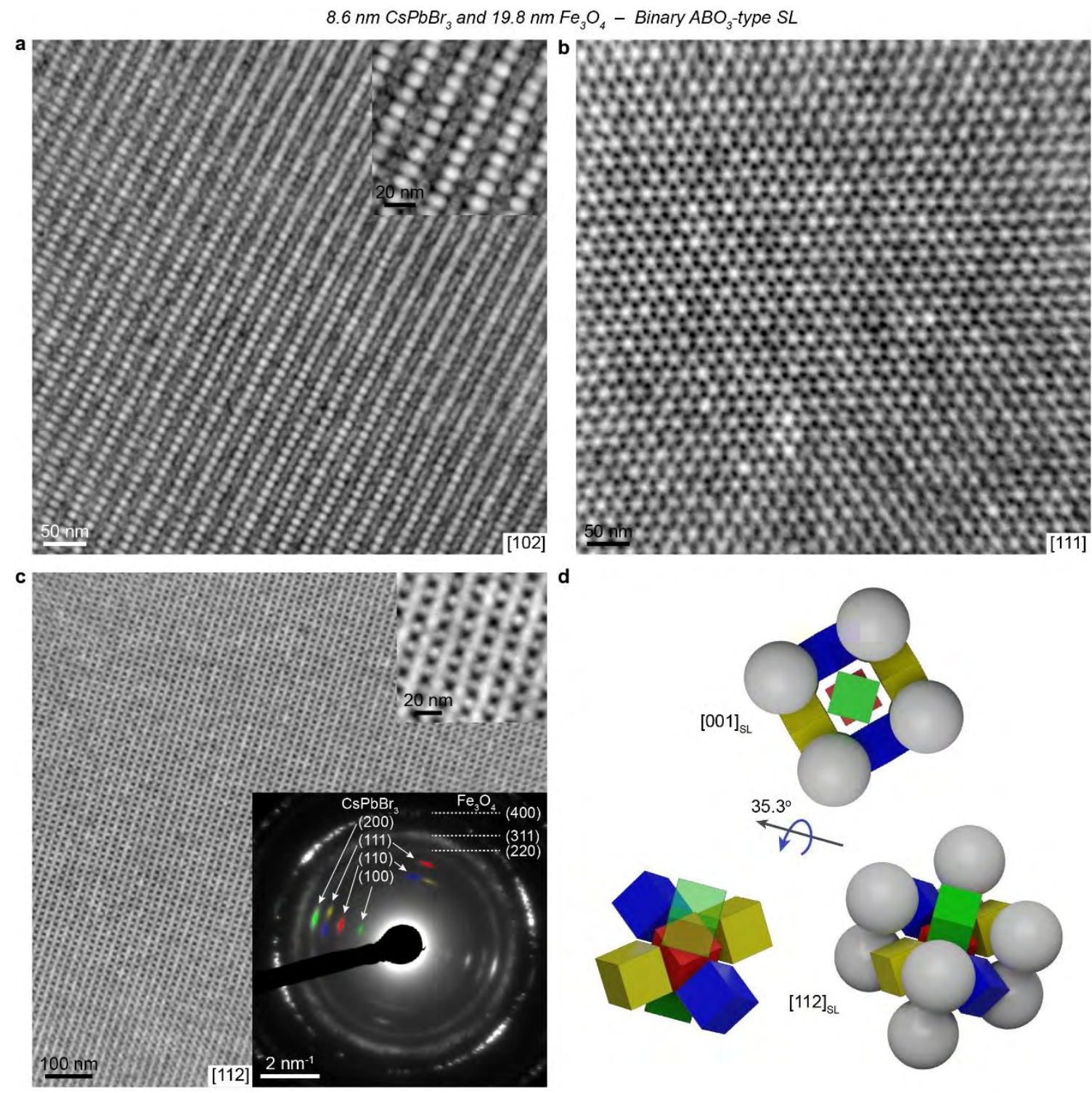

Supplementary Fig. 11 | Binary $\mathrm{ABO}_{3}$-type $\mathrm{SL}$ domains with different crystallographic orientations (8.6 nm CsPbBr 3 and $19.8 \mathrm{~nm} \mathrm{Fe} \mathrm{O}_{4} \mathrm{NCs}, \gamma=0.414$ ). a, b, c, HAADF-STEM image in, respectively, [102]SL, [111]sL, [112]sL orientations. Upper insets show higher magnification images. The bottom inset in $\mathbf{c}$ shows the ED pattern from the single domain displayed in c. The origin of the $\mathrm{CsPbBr}_{3} \mathrm{ED}$ reflections is colour-coded to match the sketch d. d, Relationship between [001]s s and [112]s projections in $\mathrm{ABO}_{3}$ lattice. [112]sL projection can be obtained by a rotation of [001]sL projection around the [110]sL axis by $\sim 35.3^{\circ}$. (111) reflection from a $\mathrm{CsPbBr}_{3}$ nanocube located on B-site (centre of the unit cell) appears in ED pattern (marked in red) since in [112]sL projection (111) lattice planes of this cube become parallel to an electron beam. The presence of diffraction spots originating from (111) lattice planes of $3 \mathrm{c}$ nanocubes (blue and yellow) which are not parallel to the electron beam in the modeled structure (form $5.6^{\circ}$ angle), may indicate a small deviation from the ideal cubic structure. 


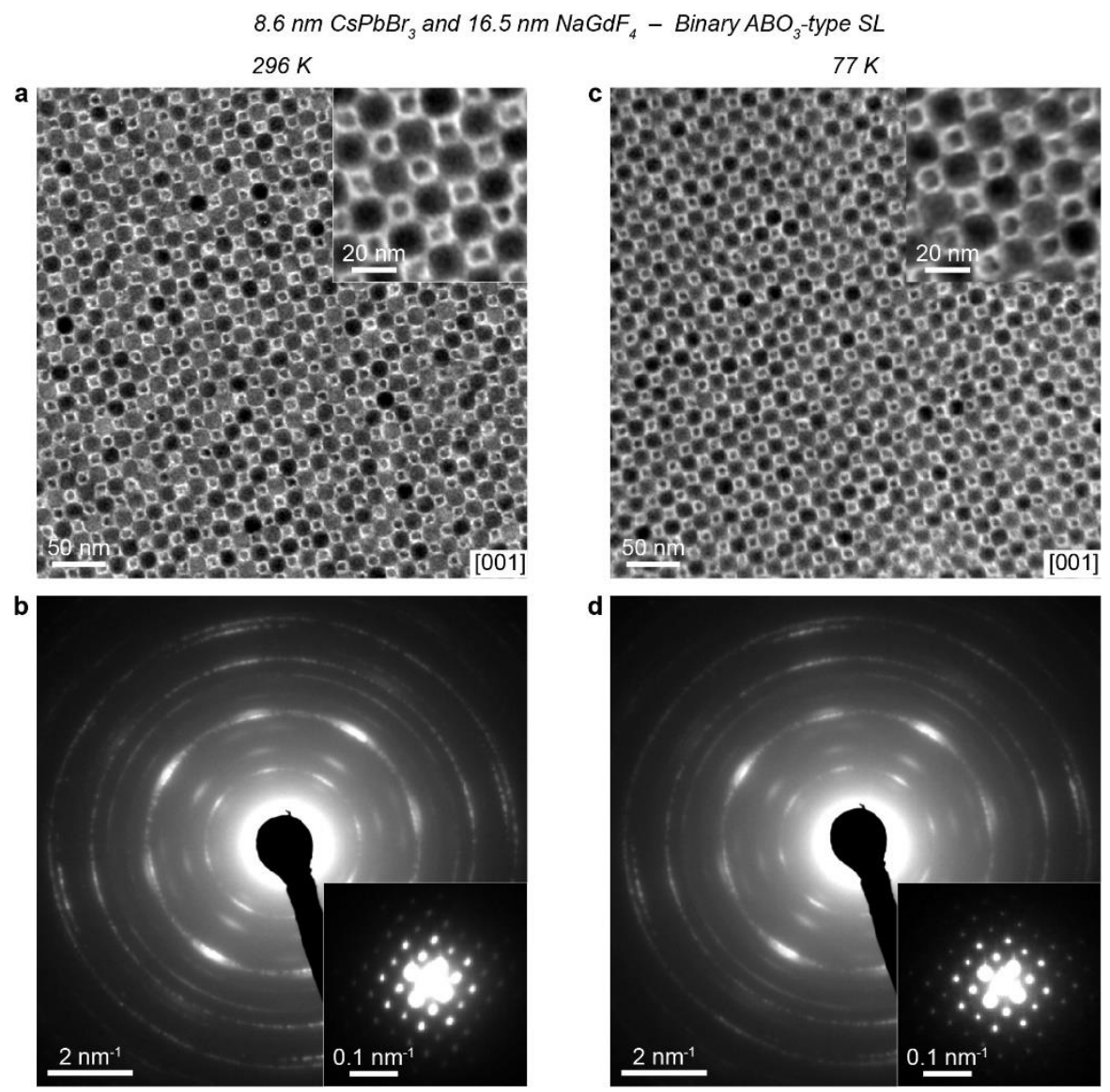

Supplementary Fig. 12 | Cryo-TEM measurements of a binary $\mathrm{ABO}_{3}$-type SL assembled from $8.6 \mathrm{~nm}$ $\mathrm{CsPbBr}_{3}$ and $16.5 \mathrm{~nm} \mathrm{NaGdF}{ }_{4} \mathrm{NCs}$ on carbon-coated TEM grid. a, TEM images and (b) corresponding wide- and small-angle (inset) ED patterns of a single SL domain in [001]sL orientation recorded at $296 \mathrm{~K}$. c, TEM images and (d) corresponding wide- and small-angle (inset) ED patterns of the same domain recorded at $77 \mathrm{~K}$. 
$\mathrm{NaCl}$-type SLs with $5.3 \mathrm{~nm} \mathrm{CsPbBr}{ }_{3} \mathrm{NCs}$

$5.3 \mathrm{~nm} \mathrm{CsPbBr}$ and $14.7 \mathrm{~nm} \mathrm{Fe}_{3} \mathrm{O}_{4}$

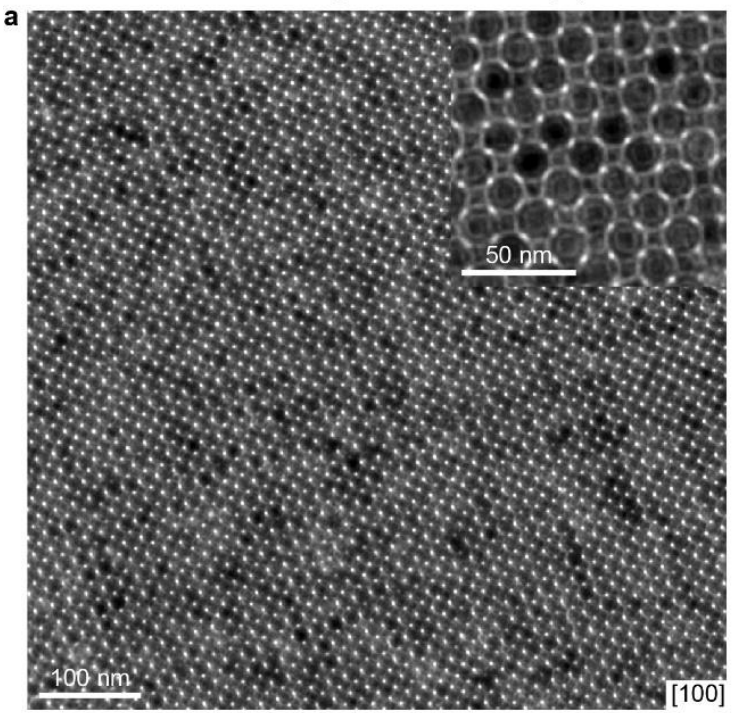

$5.3 \mathrm{~nm} \mathrm{CsPbBr}$ and $19.8 \mathrm{~nm} \mathrm{Fe}{ }_{3} \mathrm{O}_{4}$

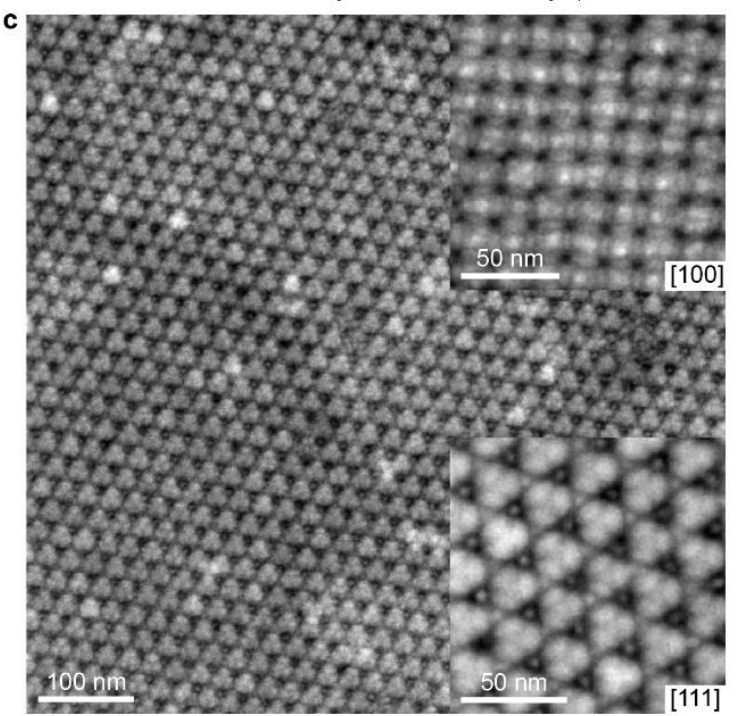

$5.3 \mathrm{~nm} \mathrm{CsPbBr}$ and $15.6 \mathrm{~nm} \mathrm{Fe} \mathrm{O}_{4}$

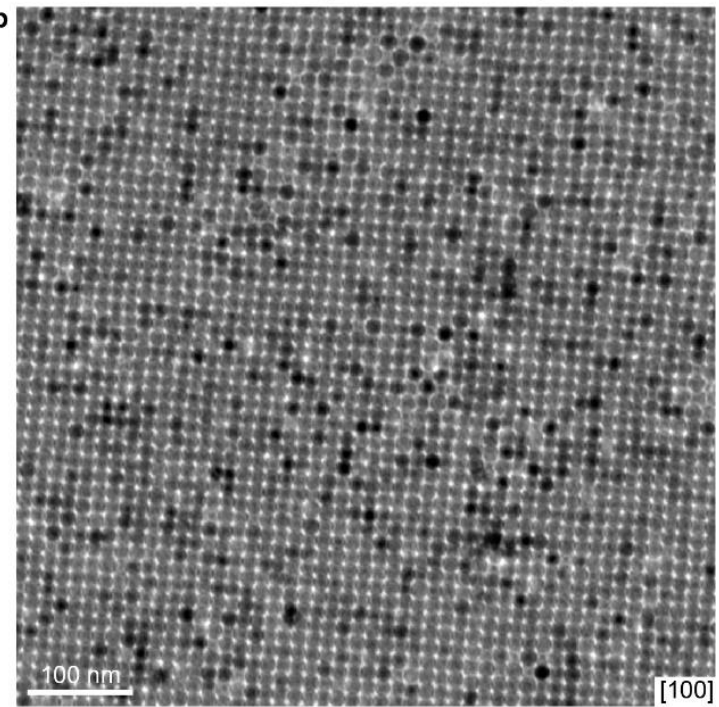

$5.3 \mathrm{~nm} \mathrm{CsPbBr}$ and $19.8 \mathrm{~nm} \mathrm{Fe} \mathrm{O}_{4} \mathrm{NaCl}$-type SL

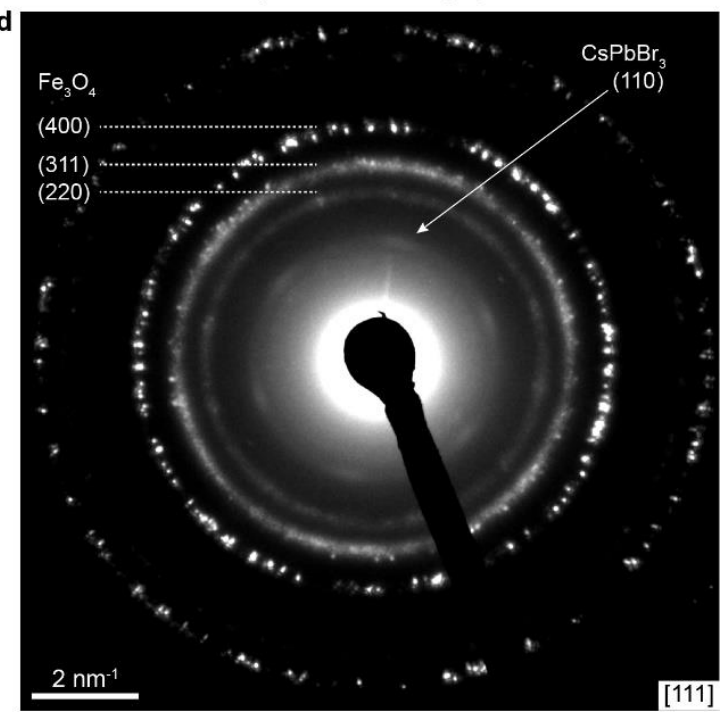

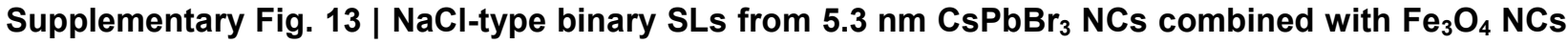
of different size. a, TEM image of a SL domain in [100]sL orientation, $\gamma=0.353$; top inset shows higher magnification image. b, TEM image of a SL domain in [100]sL orientation for $\gamma=0.336$. c, HAADF-STEM image of a SL domain in [111]sL orientation for $\gamma=0.274$; insets show higher magnification images in two typical orientations. d, ED pattern of a [111]sL-oriented domain displayed in c. Splitting of $\mathrm{CsPbBr}_{3}$ reflections from (111) lattice planes into six arcs indicates the loss of orientational freedom of nanocubes and alignment of $<100>$ directions of $\mathrm{CsPbBr}_{3} \mathrm{NCs}$ with $<100>$ sL. 
$\mathrm{NaCl}$-type SLs with $8.6 \mathrm{~nm} \mathrm{CsPbBr}{ }_{3} \mathrm{NCs}$

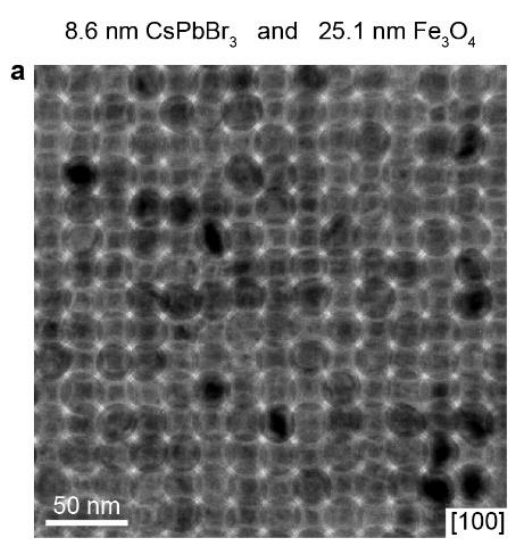

$8.6 \mathrm{~nm} \mathrm{CsPbBr}{ }_{3}$ and $19.8 \mathrm{~nm} \mathrm{Fe}_{3} \mathrm{O}_{4}$

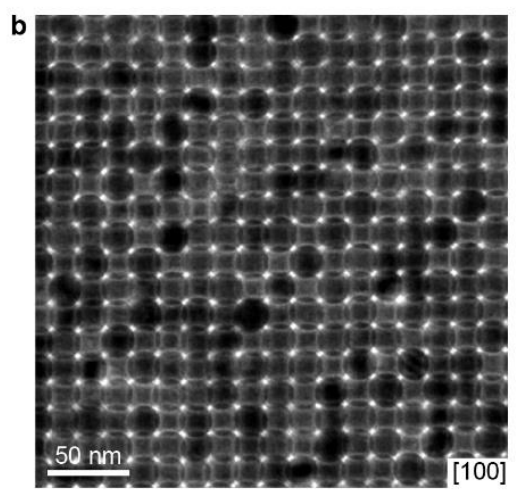

$8.6 \mathrm{~nm} \mathrm{CsPbBr}$ and $15.6 \mathrm{~nm} \mathrm{Fe}_{3} \mathrm{O}_{4}$

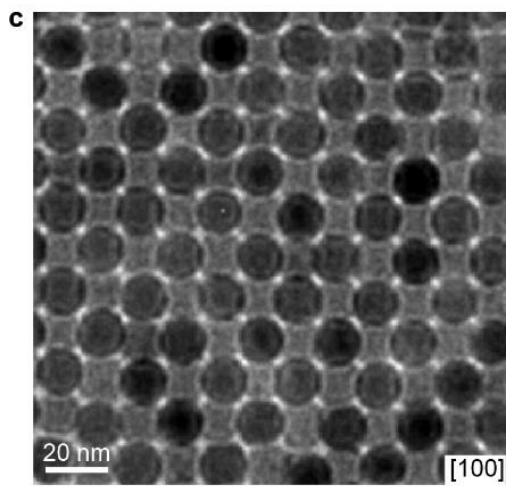

$8.6 \mathrm{~nm} \mathrm{CsPbBr}$ and $12.5 \mathrm{~nm} \mathrm{Fe}_{3} \mathrm{O}_{4}$

$8.6 \mathrm{~nm} \mathrm{CsPbBr}{ }_{3}$ and $10.2 \mathrm{~nm} \mathrm{Fe}_{3} \mathrm{O}_{4}$
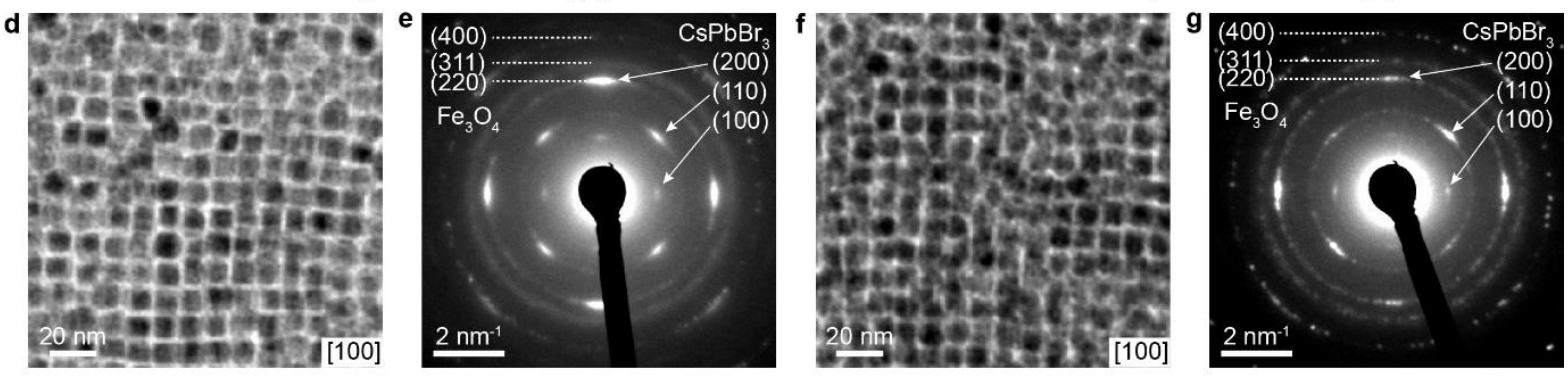

$8.6 \mathrm{~nm} \mathrm{CsPbBr}{ }_{3}$ and $18.6 \mathrm{~nm} \mathrm{NaGdF}_{4}$
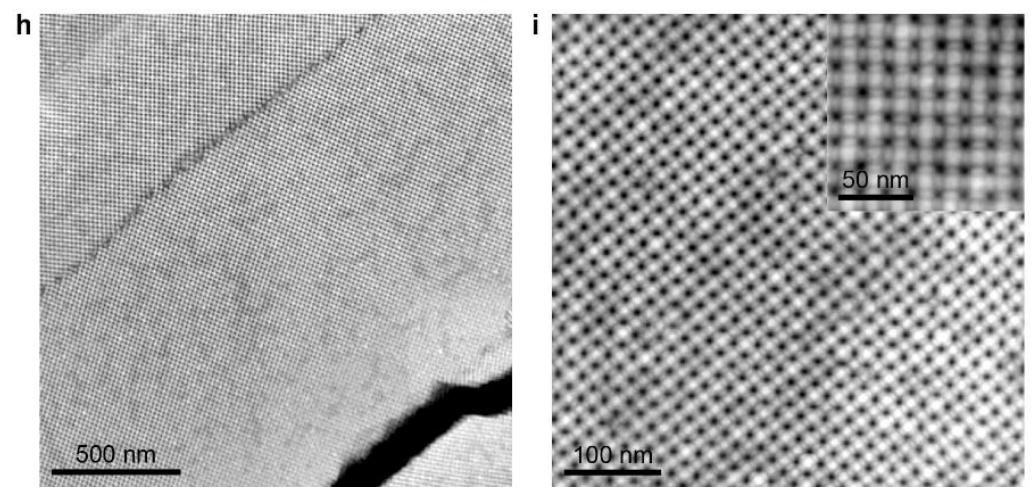

Supplementary Fig. 14 | NaCl-type binary SLs from $8.6 \mathrm{~nm} \mathrm{Cs} \mathrm{PbBr}_{3} \mathrm{NCs}$ combined with $\mathrm{Fe}_{3} \mathrm{O}_{4}$ and $\mathrm{NaGdF}_{4} \mathbf{N C s}$ of different size. a-c, d, f, TEM images of binary SL domains in [100]sL orientation for $\gamma=0.337, \gamma=0.414, \gamma=0.507, \gamma=0.612, \gamma=0.726$, respectively. $\mathbf{e , ~} \mathbf{g}$, ED patterns of SL domains displayed in $\mathbf{d}$ and $\mathbf{f}$, respectively. $\mathbf{h}, \mathbf{i}$, HAADF-STEM images at a different magnification of a binary SL in [100]SL orientation assembled from $8.6 \mathrm{~nm} \mathrm{CsPbBr} 3$ and $18.6 \mathrm{~nm} \mathrm{NaGdF}_{4} \mathrm{NCs}(\gamma=0.438)$ on a $\mathrm{Si}_{3} \mathrm{~N}_{4}$ membrane. 


\section{References:}

58. Safko J. L., P. C., Goldstein H. Classical Mechanics. (Pearson, 2001).

59. Travesset, A. Phase diagram of power law and Lennard-Jones systems: crystal phases. J. Chem. Phys. 141, 164501 (2014).

60. Anderson, J. A., Lorenz, C. D. \& Travesset, A. General purpose molecular dynamics simulations fully implemented on graphics processing units. J. Comput. Phys. 227, 5342-5359 (2008).

61. Glaser, J. et al. Strong scaling of general-purpose molecular dynamics simulations on GPUs. Comput. Phys. Commun. 192, 97-107 (2015).

62. Anderson, J. A., Eric Irrgang, M. \& Glotzer, S. C. Scalable Metropolis Monte Carlo for simulation of hard shapes. Comput. Phys. Commun. 204, 21-30 (2016).

63. Travesset, A. Soft skyrmions, spontaneous valence and selection rules in nanoparticle superlattices. ACS Nano 11, 5375-5382 (2017).

64. Ye, X., Chen, J. \& Murray, C. B. Polymorphism in self-assembled $\mathrm{AB}_{6}$ binary nanocrystal superlattices. J. Am. Chem. Soc. 133, 2613-2620 (2011).

65. Travesset, A. Topological structure prediction in binary nanoparticle superlattices. Soft Matter 13, 147-157 (2016).

66. Zha, X. \& Travesset, A. The hard sphere diameter of nanocrystals (nanoparticles). J. Chem. Phys. 152, 094502 (2020).

67. Landman, U. \& Luedtke, W. D. Small is different: energetic, structural, thermal, and mechanical properties of passivated nanocluster assemblies. Faraday Discuss. 125, 1-22 (2004).

68. Boles, M. A. \& Talapin, D. V. Many-body effects in nanocrystal superlattices: departure from sphere packing explains stability of binary phases. J. Am. Chem. Soc. 137, 4494-4502 (2015).

69. Linaburg, M. R., McClure, E. T., Majher, J. D. \& Woodward, P. M. Cs ${ }_{1-x} \mathrm{Rb}_{x} \mathrm{PbCl}_{3}$ and $\mathrm{Cs}_{1-}$ ${ }_{x} \mathrm{Rb}_{x} \mathrm{PbBr}_{3}$ solid solutions: understanding octahedral tilting in lead halide perovskites. Chem. Mater. 29, 3507-3514 (2017).

70. Cottingham, P. \& Brutchey, R. L. On the crystal structure of colloidally prepared $\mathrm{CsPbBr}_{3}$ quantum dots. Chem. Commun. 52, 5246-5249 (2016).

71. Bertolotti, F. et al. Crystal structure, morphology, and surface termination of cyan-emissive, sixmonolayers-thick $\mathrm{CsPbBr}_{3}$ nanoplatelets from X-ray total scattering. ACS Nano 13, 14294-14307 (2019).

72. Bodnarchuk, M. I. et al. Rationalizing and controlling the surface structure and electronic passivation of cesium lead halide nanocrystals. ACS Energy Lett. 4, 63-74 (2019).

73. Jun, Y. W., Lee, J. H., Choi, J. S. \& Cheon, J. Symmetry-controlled colloidal nanocrystals: nonhydrolytic chemical synthesis and shape determining parameters. J. Phys. Chem. B 109, 14795-14806 (2005).

74. Wang, $\mathrm{Y}$. et al. Shape-controlled synthesis of $\mathrm{PbS}$ nanocrystals via a simple one-step process. Langmuir 28, 16436-16443 (2012).

75. Smith, D. K., Goodfellow, B., Smilgies, D. M. \& Korgel, B. A. Self-assembled simple hexagonal $\mathrm{AB}_{2}$ binary nanocrystal superlattices: SEM, GISAXS, and defects. J. Am. Chem. Soc. 131, 32813290 (2009).

76. Miyajima, K., Kagotani, Y., Saito, S., Ashida, M. \& Itoh, T. Superfluorescent pulsed emission from biexcitons in an ensemble of semiconductor quantum dots. J. Phys. Condens. Matter 21, 195802 (2009).

77. Ashner, M. N. et al. Size-dependent biexciton spectrum in $\mathrm{CsPbBr}_{3}$ perovskite nanocrystals. ACS Energy Lett. 4, 2639-2645 (2019). 